University of Louisville

ThinkIR: The University of Louisville's Institutional Repository

Electronic Theses and Dissertations

$12-2005$

\title{
Validation of an in vivo model for monitoring trabecular bone quality changes using finite element analysis.
}

\author{
Boon Horng Kam \\ University of Louisville
}

Follow this and additional works at: https://ir.library.louisville.edu/etd

\section{Recommended Citation}

Kam, Boon Horng, "Validation of an in vivo model for monitoring trabecular bone quality changes using finite element analysis." (2005). Electronic Theses and Dissertations. Paper 721.

https://doi.org/10.18297/etd/721

This Doctoral Dissertation is brought to you for free and open access by ThinkIR: The University of Louisville's Institutional Repository. It has been accepted for inclusion in Electronic Theses and Dissertations by an authorized administrator of ThinkIR: The University of Louisville's Institutional Repository. This title appears here courtesy of the author, who has retained all other copyrights. For more information, please contact thinkir@louisville.edu. 


\title{
VALIDATION OF AN IN VIVO MODEL FOR MONITORING TRABECULAR BONE QUALITY CHANGES USING FINITE ELEMENT ANALYSIS
}

\author{
By \\ Boon Horng Kam \\ B.Sc., Mississippi State University, 1996 \\ MEng, Nanyang Technological University, 2001
}

\begin{abstract}
A Dissertation
Submitted to the faculty of the

Graduate School of the University of Louisville

In Partial Fulfillment of the Requirements

for the Degree of
\end{abstract}

Doctor of Philosophy

Department of Mechanical Engineering

Orthopedic Bioengineering Laboratory

University of Louisville

Louisville, Kentucky

December 2005 
Copyright 2005 by Boon Horng Kam

All rights reserved 


\section{VALIDATION OF AN IN VIVO MODEL FOR MONITORING TRABECULAR BONE QUALITY CHANGES USING FINITE ELEMENT ANALYSIS}

By

Boon Horng Kam

B.Sc., Mississippi State University, 1996

MEng, Nanyang Technological University, 2001

A Dissertation Approved on

$9^{\text {th }}$ November 2005

By the following Dissertation Committee:

Dissertation Director Michael J. Voor, Ph.D.

William P. Hnat, Ph.D.

William M. Pierce, Ph.D.

Robert S. Keynton, Ph.D.

Michael L. Day, Ph.D. 


\section{DEDICATION}

This dissertation is dedicated to my parents

Mr. Ah Wa Kam

and

Mrs. It Siew Wong-Kam

who have given me invaluable educational opportunities. 


\section{ACKNOWLEDGEMENTS}

I would like to thank my advisor, Dr. Michael J. Voor, for his invaluable guidance, advice and patience. I would also like to thank the other committee members, Dr. William P. Hnat, Dr. William M. Pierce, Dr. Robert S. Keynton and Dr. Michael L. Day, for their invaluable comments and advice. I would also like to thank my colleagues, Mr. Seid Waddell, Mr. Shuo Yang, and Mr. Robert Burden Jr., for their invaluable advice and assistance, Mr. Scott D. Cambron for his assistance in bone milling, and Dr. Glenn Prater Jr., head department of Mechanical Engineering, for his continuous moral support. I would also like to express my thanks to my husband, Mr. Hai Wang Tan, for his understanding and patience during those times when there was no light at the end of anything. He encouraged me and made me stick with it. Also, many thanks to my family members in Malaysia: Madam Boon Jiuan Kam, Mr. Ching Check Kam, and Mr. Boon Hee Kam for their continuous moral support. Finally, I would like to thank Madam Shu Hui Wong, Mr. and Mrs. Phun Phooi Wong, Mr. and Mrs. Soo Song Teoh. 


\title{
ABSTRACT \\ VALIDATION OF AN IN VIVO MODEL FOR MONITORING TRABECULAR BONE QUALITY CHANGES USING FINITE ELEMENT ANALYSIS
}

\author{
Boon Horng Kam \\ $9^{\text {th }}$ November, 2005
}

A combination of three techniques - high resolution micro computed tomography (micro CT) scanning, Archimedes-based volume fraction measurement and serial sectioning or milling - were used to determine the volume fraction, trabecular thickness, trabecular separation, trabecular number and micro finite element analysis combined with mechanical testing was used to determine the apparent stiffness and tissue modulus to quantify bone quality in rabbit distal femur trabecular bone. The objectives of this dissertation were two-fold. First, to develop the capabilities of micro CT scanning and micro CT image segmentation based on a slice-by-slice global thresholding technique to investigate trabecular microstructural changes in vivo and in vitro; and second, to develop the capability of translating micro CT scans into three dimensional finite element models based on direct voxel conversion technique. These results were validated within the in vivo and in vitro scans at the same time, and validated with the Archimedes-based volume fraction measurements and serial sectioning or milling experiments. The micro FE models were executed as linear analyses and the same bone cubes of the models were mechanically tested (compressive testing) to determine the correct tissue modulus of the 
bone specimens. The apparent stiffness of these micro FE models was recalculated using the average tissue modulus.

A total of six six-month-old New Zealand white rabbits were utilized in this study. Three rabbits were scanned twice in vivo seven days apart (T1 and T7) and three rabbits were only scanned once in vivo. All of the femurs were scanned in vitro. All micro CT images were obtained at $28 \mu \mathrm{m}$ (in vivo) or $14 \mu \mathrm{m}$ (in vitro) nominal resolutions. Specimens from six left and right rabbit distal femurs (medial and lateral) were measured based on Archimedes' principle and serial milling. The volume fraction for lateral condyles between two in vivo scans T1 $(0.401 \pm 0.015)$ and T7 $(0.397 \pm 0.021)$, between in vitro micro CT $(0.352 \pm 0.035)$ and Archimedes $(0.365 \pm 0.031)$ and between in vitro micro CT $(0.352 \pm 0.035)$ and serial milling $(0.369 \pm 0.031)$ were not significantly different. The medial condyles were also not significantly different: T1 $(0.513 \pm 0.010)$, $\mathrm{T} 7(0.515 \pm 0.011)$, in vitro micro CT $(0.454 \pm 0.049)$, Archimedes $(0.460 \pm 0.060)$ and serial milling $(0.467 \pm 0.050)$. Specimens from another six left and right distal femurs (medial and lateral) were mechanically tested along the anterior-posterior directions. The tissue modulus of each specimen was determined by making the calculated apparent stiffness values from FEA to be equal to mechanical compressive testing (MTS). Based on a new constant tissue modulus, the recalculated FEA apparent stiffness $(1.77 \mathrm{E} 9 \pm 6.45 \mathrm{E} 8)$ and MTS apparent stiffness $(1.76 \mathrm{E} 9 \pm 7.37 \mathrm{E} 8)$ were linearly correlated $(\mathrm{r}$-value $=0.8721)$. These findings suggest that the capabilities of slice-by-slice global thresholding and direct voxel conversion are sensitive, reliable and consistent for the study of trabecular bone microstructural changes in vivo utilizing high resolution $(\leq 28 \mu \mathrm{m})$ micro CT scanning and micro FEA. 


\section{TABLE OF CONTENTS}

PAGE

ACKNOWLEDGMENTS

ABSTRACT

LIST OF TABLES.

ix

LIST OF FIGURES

INTRODUCTION 1

BACKGROUND 5

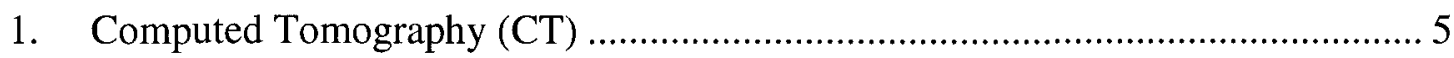

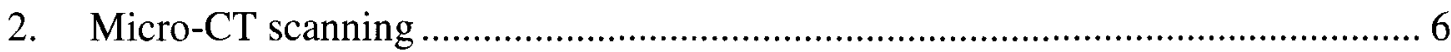

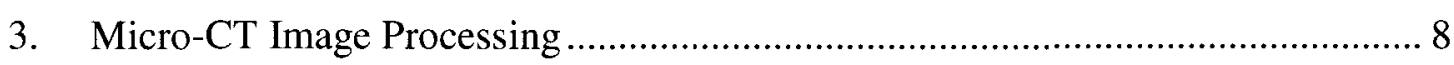

4. Micro-CT based Finite Element Analysis (Linear Elastic)................................... 10

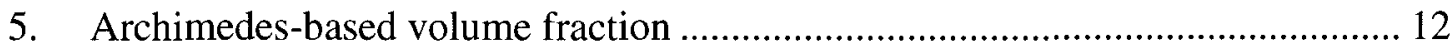

6. Serial sectioning-based volume fraction, trabecular thickness, trabecular spacing

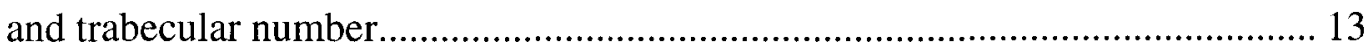

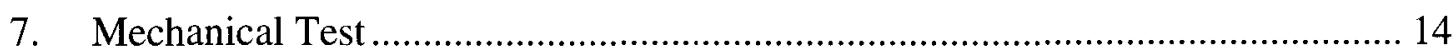

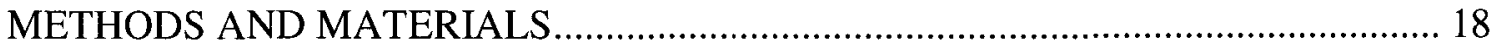

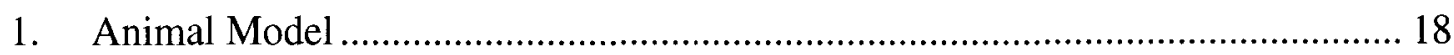

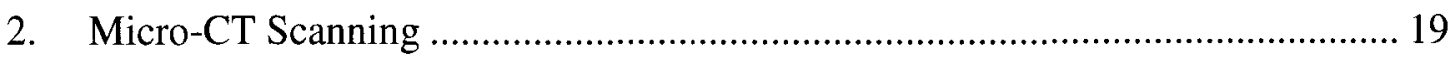

a. Scanning Hardware Description ...................................................................... 19

b. In Vivo Micro CT Scanning Procedure for a Rabbit Distal Femur .................. 21

c. In Vitro Micro CT Scanning Procedure for a Rabbit Distal Femur................... 23

2. Micro-CT Image Processing and Analysis ........................................................ 24 


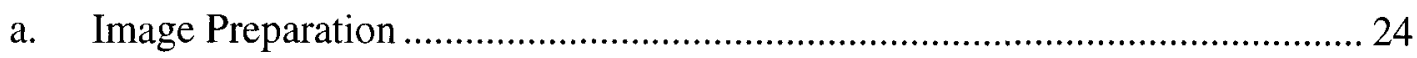

a. Thresholding Technique and Segmentation Process ………............................ 27

3. Bone Preparation Technique ............................................................................ 30

4. Archimedes'-based density and volume fraction measurement ...........................32

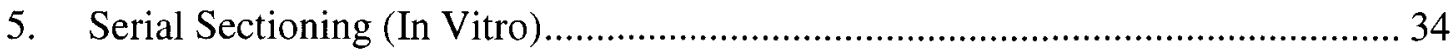

6. Linear Elastic Finite Element Analysis................................................................ 40

7. Mechanical Analysis (Compression Test) …………......................................... 43

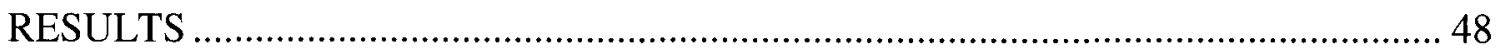

1. Micro CT: In Vivo (T1) to In Vivo (T7)......................................................... 48

2. Micro CT: In Vivo (T7') to In Vitro (Vit) ……….......................................... 51

3. Micro CT: In Vivo with correlation factor (T7'CF) ........................................5

4. Micro CT: In Vivo (T1CF) to In Vivo (T7CF) with \% correction factor............. 56

5. Archimedes, Serial Milling and Micro CT-in vitro cubes .................................... 59

6. FEA (apparent stiffness and tissue modulus) and MTS (apparent stiffness)........64

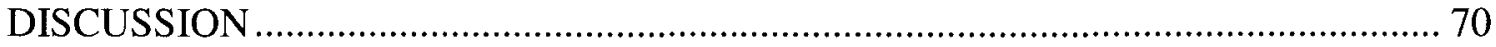

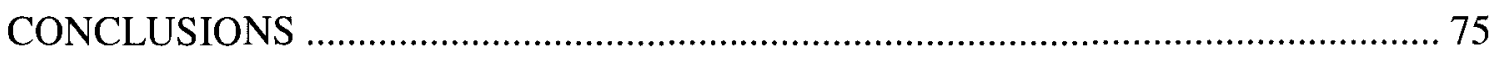

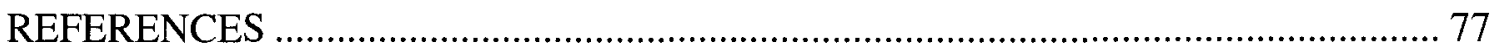

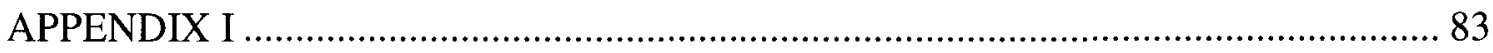

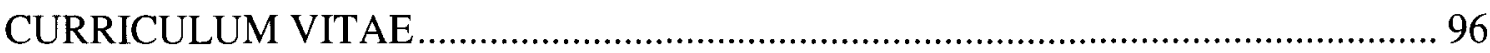




\section{LIST OF TABLES}

TABLE

PAGE

1. VF, TbTh, TbSp and TbNm for In Vivo (T1) and In Vivo (T7) .............................. 49

2. VF, Tb Th, Tb Nm and Tb Sp for In Vivo (T7') and In Vitro (Vit)..........................52

3. VF, TbTh, TbSp and TbNm for T7' with correction factor (T7'CF) compared to Vit

4. VF, $\mathrm{TbTh}, \mathrm{TbSp}$ and $\mathrm{TbNm}$ for in vivo with \% correction factor (T1CF \& T7CF).

5. Volume Fraction (VF), Trabecular Thickness (Tb Th), Trabecular Number ( $\mathrm{Tb} \mathrm{Nm}$ ) and Trabecular Spacing ( $\mathrm{Tb} \mathrm{Sp}$ ) from Archimedes-based (arc) and serial milling (SM) experiments and in vitro micro CT (CT) scans..... 61

6. FEA apparent stiffness using 12E9 (arbitrary) tissue modulus......................64

7. Tissue Modulus values of FEA based on MTS apparent stiffness......................65

8. Comparison of Apparent Stiffness Values from MTS and FEA (using average tissue modulus) 66

9. VF, Tb $\mathrm{Th}, \mathrm{Tb} \mathrm{Nm}$ and $\mathrm{Tb} \mathrm{Sp}$ of the bone cube specimens for mechanical compressive testing (MTS) and finite element analysis (FEA) 68 


\section{LIST OF FIGURES}

FIGURE

PAGE

1. Clinical CT scanner.

2. Micro-CT: micro focus X-ray tube (left), specimen manipulator (center) and detector (right) before the CCD camera (far right). 6

3. Tensile (left), bending (center) and compression (right) tests..................................... 15

4. Misalignment of the faces of bone specimens upon the platens................................. 16

5. Placement of a pivoting platen (ball bearing) to reduce the misalignment.................. 17

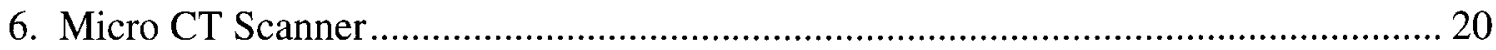

7. Rabbit Holder for in vivo scanning (left) and bone specimen holder for in vitro

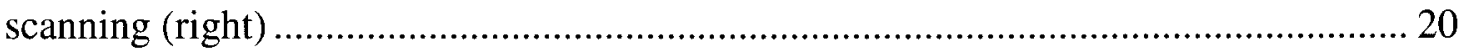

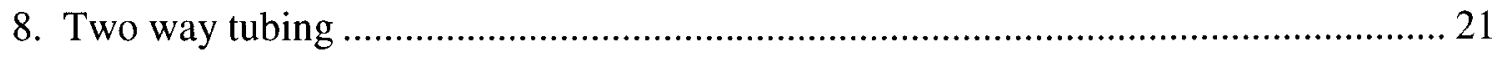

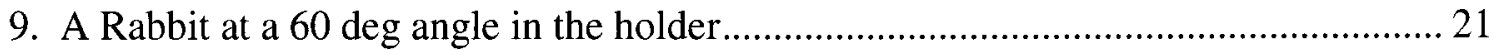

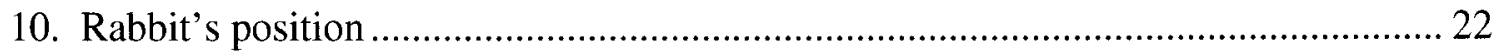

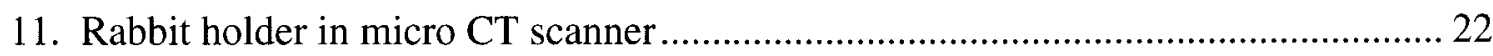

12. Tiff images (8MB) for the same rabbit scanned at different times............................25

13. Cropped $2.5 \mathrm{MB}$ tiff images for the same rabbit scanned at different times ............. 25

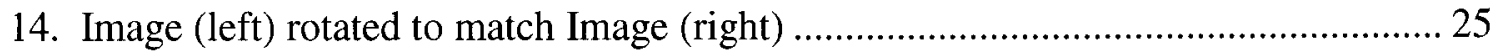

15. Cropped Images to be realigned in 3-D stacks ..................................................... 26 
16. Micro CT images - before registration (right), registration reference image (center) and after registration (left)

17. Images of $14 \mu \mathrm{m}$ (left) and degraded to $28 \mu \mathrm{m}$ (right) ........................................... 27

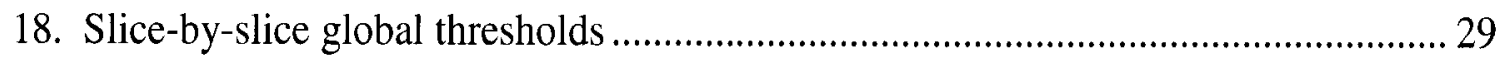

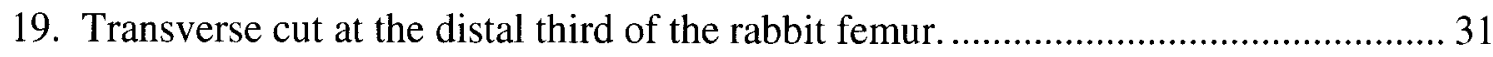

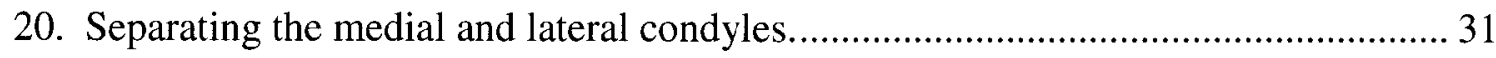

21. Proximal Condyles were fixed with adhesive on stainless steel plates. ..................... 31

22. A bone cube with posterior (violet), medial/lateral (red) and distal (green) ............. 32

23. Ultrasonic bath (left), waterpik (middle) and rinsing procedure (right) .................... 33

24. Vacuum (left), centrifuge-Microspin 24P (center), Analytical balance (right), ........ 33

25. Bone cubes exposed to $365 \mathrm{~nm} /$ long-wave UV light.................................................. 34

26. Cylindrical bone column (left) and bone mold covered with masking tape (right)... 36

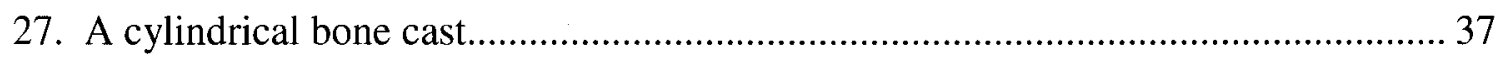

28. Micromill (left) and close up view of micromill (right)........................................... 38

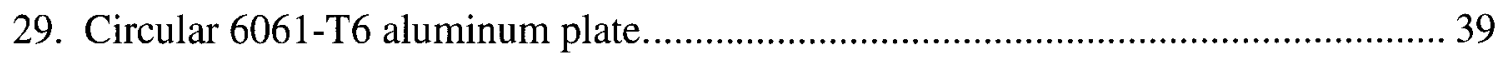

30. Serial Milling in process (left) and a serial milled image (right).............................. 40

31. A bone cube model with bone and non-bone elements. ........................................... 42

32. A bone cube model with only bone elements........................................................ 42

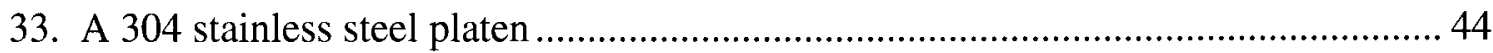

34. A close up view of a bone cube in between platens. A $25 \mathrm{~mm}$ gauge-length extensometer was attached to the platens with rubber bands 45

35. A stress-strain curve, illustrating preconditioning, apparent stiffness in the elastic region and load to failure in the plastic region. 
36. Volume of interests for $\mathrm{T} 1$ (left) and $\mathrm{T} 7$ (right) presented in three dimensional image stacks after slice-by-slice global threshold.

37. Volume fraction (VF), trabecular thickness ( $\mathrm{Tb} \mathrm{Th})$ and trabecular separation ( $\mathrm{Tb}$ Sp) of twelve bone cube specimens (medial and lateral condyles) measured in vivo (T1) were plotted against in vivo (T7). The $\mathrm{x}$ - and $\mathrm{y}$ - axes are unitless for VF and $\mathrm{mm}$ for $\mathrm{Tb} \mathrm{Th}$ and $\mathrm{Tb} \mathrm{Sp}$.

38. Lateral-medial $(\mathrm{T} 1 \& \mathrm{~T} 7)$ average values shown with standard deviation $(\mathrm{n}=12)$ for volume fraction (VF), trabecular thickness (Tb $\mathrm{Th}$ ) and trabecular separation (Tb Sp) of rabbit distal femur. The $\mathrm{y}$-axis is unitless for $\mathrm{VF}$ and $\mathrm{mm}$ for $\mathrm{Tb} \mathrm{Th}$ and $\mathrm{Tb} \mathrm{Sp} . .50$

39. Volume of interests for T7' (left) and Vit (right) presented in three dimensional image stacks after slice-by-slice global threshold. 52

40. Lateral-medial (T7' vs. Vit) average \% differences shown with standard deviation (n = 12) for volume fraction (VF), trabecular thickness $(\mathrm{Tb} \mathrm{Th})$, trabecular number $(\mathrm{Tb}$ $\mathrm{Nm})$ and trabecular separation $(\mathrm{Tb} \mathrm{Sp}) .(*$ significantly different for $\mathrm{p}<0.05 \mathrm{t}-\mathrm{Tests}$ : Paired Two Sample for Means) 53

41. Volume fraction (VF), trabecular thickness ( $\mathrm{Tb} \mathrm{Th}$ ) and trabecular separation ( $\mathrm{Tb} \mathrm{Sp}$ ) of twelve bone cube specimens (medial and lateral condyles) measured in vivo (T7') were plotted against in vitro (Vit). The $\mathrm{x}$ - and $\mathrm{y}$ - axes are unitless for VF and $\mathrm{mm}$ for Tb Th and Tb Sp.

42. Volume fraction (VF), trabecular thickness ( $\mathrm{Tb} \mathrm{Th}$ ) and trabecular separation ( $\mathrm{Tb} \mathrm{Sp}$ ) of twelve bone cube specimens (medial and lateral condyles) measured in vivo with $\%$ correction factor $\left(\mathrm{T} 7^{\prime} \mathrm{CF}\right)$ were plotted against in vitro (Vit). The $\mathrm{x}$ - and $\mathrm{y}$-axes are unitless for $\mathrm{VF}$ and $\mathrm{mm}$ for $\mathrm{Tb} \mathrm{Th}$ and $\mathrm{Tb} \mathrm{Sp}$ 
43. Lateral-medial (in vivo-T7' vs. in vitro-Vit) and lateral-medial (in vivo with correction factor-T7'CF vs. in vitro-Vit) average \% differences shown with standard deviation $(\mathrm{n}=12)$ for volume fraction $(\mathrm{VF})$, trabecular thickness $(\mathrm{Tb} T \mathrm{Th})$, trabecular number $(\mathrm{Tb} \mathrm{Nm})$ and trabecular separation $(\mathrm{Tb} \mathrm{Sp}) .(*$ = significantly different for p $<0.05$ t-Tests: Paired Two Sample for Means) The VF, Tb Th, Tb Nm and Tb Sp for the lateral and medial condyles between $\mathrm{T} 7^{\prime} \mathrm{CF}$ and. Vit were not significantly different $(\mathrm{p}=0.36, \mathrm{p}=0.85, \mathrm{p}=0.72 \& \mathrm{p}=0.84, \mathrm{p}=0.17, \mathrm{p}=0.34, \mathrm{p}=0.33 \& \mathrm{p}=$ $0.40)$. 56

44. Volume fraction (VF), trabecular thickness ( $\mathrm{Tb} \mathrm{Th}$ ) and trabecular spacing ( $\mathrm{Tb} \mathrm{Sp}$ ) of twelve bone cube specimens (medial and lateral condyles) measured in T1CF is plotted against T7CF. Correlation of VF between T1CF and T7CF was expected to be perfect while the $\mathrm{Tb} \mathrm{Sp}$ and $\mathrm{Tb} \mathrm{Th}$ between $\mathrm{T} 1 \mathrm{CF}$ and T7CF were highly correlated. The $\mathrm{x}$ - and $\mathrm{y}$ - axes are unitless for $\mathrm{VF}$ and $\mathrm{mm}$ for $\mathrm{Tb} \mathrm{Th}$ and $\mathrm{Tb} \mathrm{Sp} . . . . .58$ 45. Lateral-medial (in vivo with correction factor-T1CF\&T7CF vs. in vitro-Vit) averages are shown with standard deviation $(\mathrm{n}=12)$ for volume fraction $(\mathrm{VF})$, trabecular thickness $(\mathrm{Tb} \mathrm{Th})$ and trabecular separation $(\mathrm{Tb} \mathrm{Sp}) .{ }^{*}=$ significantly different for $\mathrm{p}<0.05$ T-Tests: paired two sample for means) The $y$ - axis is unitless for VF and $\mathrm{mm}$ for $\mathrm{Tb} \mathrm{Th}$ and $\mathrm{Tb} \mathrm{Sp}$. The VF and Tb Sp for lateral and medial condyles between T1CF\&T7CF and Vit were not significantly different $(p=0.429, p=0.602, p=0.148 \&$ $\mathrm{p}=0.107)$. The $\mathrm{Tb}$ Th for lateral condyles between T1CF\&T7CF and Vit were not significantly different $(\mathrm{p}=0.193$ ) but significantly different for medial condyles $(\mathrm{p}=0.027)$ 58 
46. Three dimensional image stacks of CT (left) and SM (right) from the same bone cube after slice-by-slice global threshold.

47. Average values of volume fraction (VF) for lateral and medial among Archimedes (arc), serial milling (SM) and in vitro micro CT (CT) are shown with standard deviation $(n=11)$ The coefficient of variation in VF between Arc and SM ranged between $0.10 \%$ and $0.22 \%$. The VF for lateral and medial condyles between CT and Arc and between CT and SM were not significantly different $(p=0.294, p=0.649, p$ $=0.115, \mathrm{p}=0.118)$. The $\mathrm{y}$ - axis is unitless for VF.

48. Correlation of volume Fraction (VF) for eleven bone cube specimens (medial and lateral condyles) in three groups (SM-arc, CT-arc \& SM-CT) were shown. The $\mathrm{x}$ and $\mathrm{y}$ - axes are unitless for VF.

49. Trabecular Thickness ( $\mathrm{Tb} \mathrm{Th}$ ) and Trabecular Separation ( $\mathrm{Tb} \mathrm{Sp}$ ) for lateral and medial condyles between serial milling (SM) and in vitro micro CT (CT) bone cubes specimens are shown with standard deviation $(\mathbf{n}=11)\left(^{*}=\right.$ significantly different for $\mathrm{p}<0.05$ t-Tests: Paired Two Sample for Means) The Tb Th for lateral and medial condyles and $\mathrm{Tb} \mathrm{Sp}$ for lateral condyles between SM and CT were significantly different $(\mathrm{p}=0.011, \mathrm{p}=0.047, \mathrm{p}=0.013)$ but the $\mathrm{Tb} \mathrm{Sp}$ for lateral and medial condyles and $\mathrm{Tb}$ Nm for medial condyles between SM and CT were not significantly different $(\mathrm{p}=0.181, \mathrm{p}=0.877, \mathrm{p}=0.207)$. The $\mathrm{y}$ - axis is $\mathrm{mm}$ for $\mathrm{Tb} \mathrm{Th}$ and $\mathrm{Tb} \mathrm{Sp}$.

50. Correlation of trabecular thickness ( $\mathrm{Tb} \mathrm{Th}$ ) and trabecular separation ( $\mathrm{Tb} \mathrm{Sp}$ ) for eleven bone cube specimens (medial and lateral condyles) between SM and CT were reported. The $\mathrm{x}$ - and $\mathrm{y}$ - axes are $\mathrm{mm}$ for $\mathrm{Tb} \mathrm{Th}$ and $\mathrm{Tb} \mathrm{Sp}$. 63 
51. Force distribution of an FEA model

52. Apparent Stiffness of twelve rabbit distal femur bone cube specimens (medial and lateral condyles) measured in nondestructive mechanical testing (FEA) were plotted against mechanical testing experiment (MTS)

53. FEA Apparent stiffness of twelve rabbit distal femur bone cube specimens (medial and lateral condyles) were plotted against volume fraction (VF), trabecular thickness ( $\mathrm{Tb} \mathrm{Th}$ ), and trabecular separation ( $\mathrm{Tb} \mathrm{Sp}$ ). The $\mathrm{X}$-axis is $\mathrm{Pa}$ for apparent stiffness while the $\mathrm{y}$-axis is unitless for VF, $\mathrm{mm}$ for $\mathrm{Tb} \mathrm{Th}$ and $\mathrm{Tb} \mathrm{Sp}$.

54. MTS Apparent stiffness of twelve rabbit distal femur bone cube specimens (medial and lateral condyles) were plotted against volume fraction (VF), trabecular thickness ( $\mathrm{Tb} \mathrm{Th}$ ), and trabecular separation $(\mathrm{Tb} \mathrm{Sp})$. The $\mathrm{x}$-axis is $\mathrm{Pa}$ for apparent stiffness while the $\mathrm{y}$-axis is unitless for $\mathrm{VF}, \mathrm{mm}$ for $\mathrm{Tb} \mathrm{Th}$ and $\mathrm{Tb} \mathrm{Sp}$. 69 


\section{INTRODUCTION}

Bone is predominantly a load bearing tissue consisting of two types, cortical bone and cancellous bone. Cortical bone is a dense and solid mass while cancellous bone is composed of a lattice of plates and rods known as trabeculae. The interstices between the trabeculae are filled with marrow. Cancellous bone is also referred to as trabecular or spongy bone. Trabecular bone is found at the ends of all long bones and within flat and irregular bones, such as the sternum, pelvis and spine. Trabecular bone distributes mechanical loads from the articulating joints to more dense cortical bone of the shaft in long bones and trabecular bone also acts as a main load-carrying component in vertebral bodies. Trabecular bone only represents $20 \%$ of the total bone mass, but it makes up approximately $67 \%$ of the total bone surface (Jee, 2001).

In a bone, the cellular activity takes place predominantly on bone surfaces through the action of bone forming cells (osteoblasts) and bone resorbing cells (osteoclasts). Since trabecular bone has such a large surface area, it is more highly subjected to the effects of cell mediated diseases such as osteoporosis than cortical bone. Because of its lattice structure, loss of bone mass in trabecular bone can lead to dramatic reductions in bone strength or quality, thus leading to bone fracture. In studies of bone as it relates to diseases such as osteoporosis and various medical treatments for these diseases, it is necessary to measure the quality of the trabecular bone being studied. Because bone is load bearing, the single best measure of its quality is its strength. 
It is not always possible to measure a bone's strength directly, so many indirect methods have been developed. A simple and basic measurement is apparent density or volume fraction (bone volume/total volume: BV/TV) because those values strongly correlate with mechanical properties. An accepted method for obtaining the true density or volume fraction of a bone cube measurement relies on the principle of Archimedes. However, these measurements do not directly relate to the structural arrangement of bone. Volume fraction only describes the amount of bone tissue within a given volume.

Another class of bone quality measurements includes the structural characteristics such as the trabecular thickness ( $\mathrm{Tb} \mathrm{Th}$ ), spacing ( $\mathrm{Tb} \mathrm{Sp}$ ), number ( $\mathrm{Tb} \mathrm{Nm}$ ) and orientation. A traditional method to look at the structural characteristics of trabecular bone is based on 2-D histological sections. When a bone cube is repeatedly sectioned and images automatically captured by a high resolution digital camera, the structural characteristics of trabecular bone can be three-dimensionally viewed in an image stack. This traditional, but proven, method for capturing high resolution digital images of bone cubes is referred to as serial sectioning. It is important that the structural characteristics of trabecular bone are considered because bone is known to remodel its threedimensional architecture in response to mechanical load (Wolff, 1892). Some trabecular structures have a very pronounced preferred orientation while others do not. The presence of trabecular structure in a preferred orientation indicates its mechanical anisotropy (different mechanical properties when loaded along different axes). The bone is stiffer in its preferred direction and less stiff in the other directions.

The best measurement of bone quality is made by looking directly at its strength or at least its elastic properties which are highly correlated with strength. The stiffness values 
of trabecular bone can be measured using traditional mechanical testing. However, it is better if the stiffness values are obtained nondestructively. Mechanical testing for strength requires destruction of the bone specimen being tested. Mechanical testing for stiffness can preserve the specimen, but creation of the test specimen requires permanently altering the intact bone. The only way to evaluate bone strength or stiffness in a truly nondestructive manner is by using a virtual mechanical test or computational simulation in the form of finite element analysis (FEA).

The ideal situation would be to find a method which could not only be used to three dimensionally capture trabecular bone microstructure from a bone specimen, but also track the changes of trabecular micro architecture within a living animal. High resolution micro-CT which has proven its ability to capture detailed images of the micro architecture of trabecular bone is the chosen research tool for this study. Not only is producing a reliable and repeatable high resolution micro-CT image important, but an image segmentation technique also needs to be developed that is capable of extracting all bone material while deleting all non-bone material in a micro-CT image. The next challenge was to develop the capability of translating bone information from a micro CT image into a finite element model. Therefore, the capability of using a direct conversion technique for translating bone information from a micro $\mathrm{CT}$ image into a finite element model needed to be developed.

The objective of this dissertation was to develop the capability to investigate the trabecular microstructural changes in a rabbit distal femur in vivo (within a living animal) and in vitro (in glass - outside a living animal) utilizing a combination of high resolution 
micro computed tomography (micro, CT) and finite element analysis (FEA). The hypotheses of this dissertation include the following:

(1) High resolution micro CT which has proven its ability to capture and produce high resolution digital images of trabecular bone used in this dissertation is within an accepted accuracy when validated with traditional experimental methods of Archimedesbased volume fraction and serial milling to measure bone structural characteristics.

(2) Micro finite element analysis, a nondestructive analysis, used in this dissertation is within an accepted accuracy when validated with traditional mechanical compressive testing.

The significance of this study demonstrates a research tool that can be used to study the effects of disease and disease treatments of trabecular bone quality in a way that has not been done before. This model will allow the study of changing micro architecture and mechanical properties in a living animal. The size of the rabbit model allows the analysis to be done on a sufficient volume of bone so that conclusions can be drawn about trabecular bone tissue in general. 


\section{BACKGROUND}

\section{Computed Tomography (CT)}

According to the American Heritage Dictionary, $3^{\text {rd }}$ edition (1994), tomography is a technique for making detailed images of a predetermined plane section of a solid object. Computer-assisted tomography (CT) typically refers to $\mathrm{x}$-ray radiography in which a three-dimensional image of a body structure is constructed by computer from a series of plane cross-sectional images made along an axis. A standard hospital-based CT system is composed of two components; mainly the X-ray tube as a source on one side of the patient and a 2D detector on the opposite side. The rotating source-detector is used to scan a supine patient that is indexed along the axis to create the $3-\mathrm{D}$ image data set (Figure 1). The source-detector geometry is fixed with a certain length such that nearly all patients, including large patients, can be scanned. The in-plane resolution is limited mainly by the source-detector geometry, the source-object geometry and the size of the object itself. Clinical CT generally provides in-plane resolution of $1 \mathrm{~mm}(1000 \mu \mathrm{m})$.

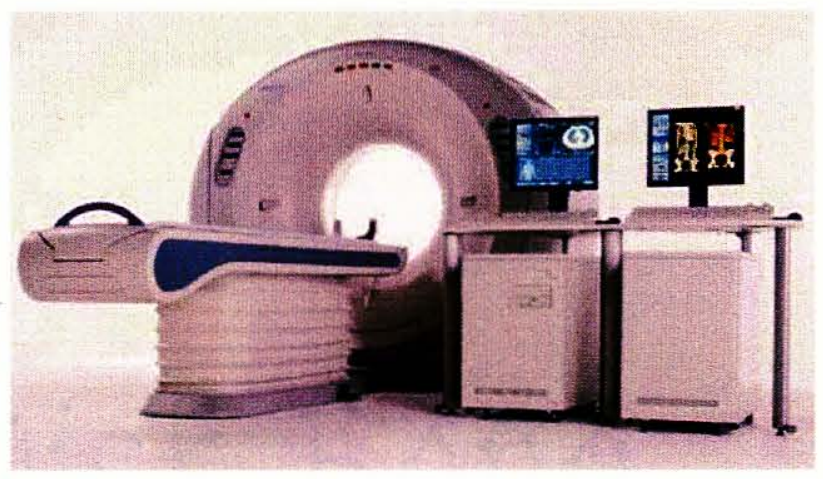

Figure 1. Clinical CT scanner. 
2. Micro-CT scanning

The general micro-CT system is similar to clinical CT except that it has a rotating and translating specimen manipulator rather than a rotating source-detector. The sourceimage-detector geometry is also more compact to achieve higher image resolution. The micro-CT was pioneered by Feldkamp et al. (1989). It was used with a micro-focus Xray tube as a source, an image intensifier as a $2 \mathrm{D}$ detector, and a cone-beam reconstruction algorithm to create a $3 \mathrm{D}$ object with a much higher in-plane resolution (5 $\mu \mathrm{m}$ to $100 \mu \mathrm{m}$ ) compared to clinical CT (Figure 2). The specimen is always positioned as close as possible to the source to give maximum magnification of the object.

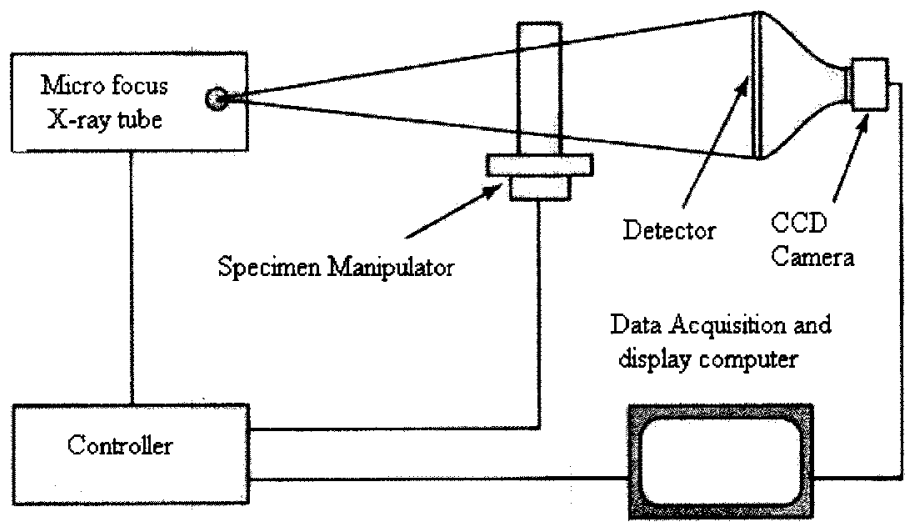

Figure 2. Micro-CT: micro focus X-ray tube (left), specimen manipulator (center) and detector (right) before the CCD camera (far right).

In the interest of diagnosing or preventing problems associated with bone quality, a non-invasive assessment of bone measurement is preferred. It is important to analyze the trabecular micro-architecture because the mechanical properties of the trabecular bone are dependent on its micro-architecture. Current methods for non-invasive assessment of bone are primarily based on plain 2-D X-ray, relatively low resolution clinical CT or bone densitometry using methods such as dual energy x-ray 
absorptiometry (DEXA). However, these methods cannot detect microstructural changes in trabecular bone because the trabecular structure in the size range of less than $200 \mu \mathrm{m}$ cannot be seen with these bone densitometry measurements which are on the order of 1 $\mathrm{mm}$ resolution. Relatively recent development of pQCT (peripheral quantitative computed tomography), micro-MRI and micro-CT have been used to visualize the internal trabecular micro architecture and better assess bone quality. These methods have been used to assess trabecular bone micro-structure in vitro using pQCT and micro-MRI with a resolution of 150 to $500 \mu \mathrm{m}$ and using micro-CT with a resolution of less than $50 \mu \mathrm{m}$ (Feldkamp et al., 1989; Kuhn et al., 1990; Bonse et al., 1994; Ruegsegger et al., 1996; Hildebrand et al. 1997, Van Rietbergen et al., 1998; Mueller et al., 1998; Engelke et al., 1993).

Only when the scanning can be done in vivo can we take advantage of the nondestructive nature of micro-CT. This provides the possibility of assessing the mechanical properties or fracture risk of a volume of bone at different points in time (Genant et al., 1999; van Rietbergen et al., 2002) in the same subject. The rat has been an attractive model in the study of trabecular microstructure (Kinney et al., 1995; Kinney et al., 1998; Lane et al., 1998; Stenstrom et al., 1995). However, the limitation of using a rat model is the restricted trabecular bone volume that can be obtained to meet the continuum assumption of approximately 3 to $5 \mathrm{~mm}$ in each direction (Harrigan et al., 1988). Even if this size of bone cube exists in the rat, the boundaries of the cube would be very close to the surface or cortices of the bone. As a result, it would be difficult to isolate a trabecular bone cube that is large enough to meet this requirement. 


\section{Micro-CT Image Processing}

In micro-CT image processing, it is important to distinguish bone material from nonbone material. When given a bone image, consisting of cortical and trabecular bone, at its grey level, the grey level of bone voxel can be represented in a range between 1 to 255 if it is an 8-bit tiff image and 1 to 65535 if it is a 16-bit tiff image. The grey level represents the density of bone. In the segmentation process, there are two types of thresholding: (1) global thresholding and (2) local thresholding. A global/single thresholding is one in which one number (grey scale value) is selected, above which all pixels are considered bone and below which all pixels are considered non-bone. This method is usually based on the Otsu automatic algorithm (Otsu, 1979) or based on other image segmentation algorithms that have been used on pQCT, MR, CT and micro-CT images (Helterbrand et al. 1997; Sivewright et al., 1994; van Rietbergen et al., 1998) when analyzing a region of only trabecular bone. The segmentation algorithm used on pQCT images was based on first analyzing the histogram of pixel intensities from a bone image (Helterbrand et al, 1997). The histogram contained a group of intensity levels attributed to the background and a group of intensity levels attributed to the bone. The intensity level representing bone was used to produce a binary image. Another image segmentation algorithm used on MR or CT images was based on the mean of intensity level representing bone in a region of interest (Sivewright et al., 1994). Other image segmentation algorithms used on micro-CT, $\mathrm{pQCT}$ and MR images based on a singlethreshold value were also reported where the value of a threshold was set based on the comparison of bone volume fraction from $\mathrm{pQCT}$ system to MR system to micro-CT system (van Rietbergen et al., 1998). Of these thresholding techniques, the global 
thresholding technique based on Otsu algorithm proved to be valid on micro-CT images which regions of trabecular bone are concerned (Uchiyama et al., 1997).

Local thresholding is based on the calculation, within small neighborhoods throughout the volume, such that each voxel can be thresholded optimally. This should be a better method of segmentation when a large difference of grey level exists in an image, i.e. a combination of cortical and trabecular bone. A general local thresholding method for micro-CT based on the so-called maximum height $(\mathrm{HMH})$ protocol has been proposed (Kuhn et al., 1990; Elmoutaouakkil et al., 2002). As a summary, voxels are considered bone if their CT number is higher than one-half the difference between local minima (background) and local maxima (bone) in which the local threshold reflects the density levels in a local neighborhood of pixels about the point of interest. Another local thresholding algorithm was developed for CT images (Dufresne, 1998). This algorithm was used to compensate for beam-hardening effects based on the analysis of the grey level histogram of bone voxels. It calculates the two largest peaks representing bone in the histogram and if the higher grey level peak is at least twice the value of the other grey level peak, then the local threshold can be determined as an average of the two peak values. Otherwise an average threshold representing bone voxels was used as the estimate for the threshold value. Other local thresholding methods for micro-CT are based on standard edge detection algorithms which calculate the local threshold value of a bone voxel using the detection of the bone surfaces and edges within its neighborhood. Subsequently, the grey value of each bone voxel is compared with its local threshold value to determine the voxel as bone when the grey value is higher than the threshold value and designate it as non-bone otherwise (Waarsing et al., 2004). 
In summary, the various local thresholding methods of high resolution scans have resulted in accurate representation of the volume. However, when the resolution of the scans was decreased, the local thresholding methods started to overestimate the volume of the scan, a phenomenon referred to as smearing of the thin structures (Kuhn et al., 1990; Dufresne, 1998; Elmoutaouakkil et al., 2002; Waarsing et al., 2004). When good quality scans were made at high resolution and the samples have a homogenous structure, i.e. only containing the trabeculae region of interest, a global thresholding method performs just as well as the local thresholding technique (Waarsing et al., 2004).

\section{Micro-CT based Finite Element Analysis (Linear Elastic)}

Finite element models that describe trabecular structure were first developed under the assumption that it is a repetitive structure (Gibson, 1985; Hollister et al., 1991). However, the accuracy and applicability of these numerical methods is only an approximation and not useful to study specific samples because such an idealized repetitive structure is very rare for trabecular bone. With $\mathrm{CT}$, there is complete 3-D information in the form of voxels, which can be turned directly into finite elements. Since micro-CT could represent the trabecular structure on a micro level; meshing methods in FEA to represent micro-CT images have been developed to automate the complex FE-models. Fyhrie and Hamid (1993) and Hollister et al.(1994) have used voxel data sets to construct detailed FE models of small pieces (up to $2.3 \mathrm{~mm}$ cubes) of trabecular bone. Another attempt was made using the marching cube algorithm (Lorensen and Cline, 1987) to create finite element models of constant density surfaces from 3D medical data and to provide meshes with tetrahedral elements (Frey et al., 1994; Müller and Rüegsegger, 1995). In addition to the above mentioned attempts, another FE 
strategy based on direct voxel conversion technique was also developed (Van Rietbergen et al., 1995). This method was applied on rectangular voxels from digitized crosssectional images using a special FEA solver. These rectangular voxels were directly converted to equally sized eight-node brick elements to enable a full, realistic threedimensional analysis. The above mentioned FE models have demonstrated the possibility of obtaining tissue modulus and the elastic properties (stiffness) of trabecular bone. Being able to incorporate the direct voxel conversion technique from micro CT-based images to micro FEA into our experiments with living rabbits, the mechanical integrity of trabecular bone could be virtually evaluated at different times in the same animal.

However, the above micro FE models described were only limited to in vitro micro CT images scanned at resolutions between $40 \mu \mathrm{m}$ to $100 \mu \mathrm{m}$. It is however important to understand that the micro FE models should be based on the order of $20 \mu \mathrm{m}$ resolution micro CT images although a resolution of $30 \mu \mathrm{m}$ micro CT images is still acceptable (Ladd and Kinney, 1998). When a high resolution micro CT image is degraded for a practical finite element analysis, it is an image which still contains the detailed trabecular structure for analysis. When a low resolution micro CT image is degraded, it is an image which already does not contain detailed trabecular structure for analysis. It is also important to understand that the high resolution micro CT images can only be degraded to a third of the average trabecular thickness which is approximately $60 \mu \mathrm{m}$ as oppose to an average original trabecular thickness of $180 \mu \mathrm{m}$ because the FE model results can still describe the trabecular structure (in particular its volume fraction) reasonably well (Van Rietbergen et al., 1995). Another disadvantage of performing micro CT scans of trabecular bone at lower resolutions is the limitation of not being able to accurately 
determine its trabecular thickness (Kothari et al., 1998) because trabecular thickness measurements show a strong dependency on resolution in order to obtain precise measurements. The thickening of the vertical trabeculae and the loss of horizontal trabeculae was seen in degraded micro CT images from $40 \mu \mathrm{m}$ to $100 \mu \mathrm{m}$.

\section{Archimedes-based volume fraction}

It is generally important to use an independent and proven accurate method for measuring density and volume fraction. A classical method for determining density based upon Archimedes' principle (law of buoyancy) was developed in the third century B.C. Archimedes' principle dictates that the density of a bone sample ( $\left.\rho_{\text {bone }}\right)$ can be determined from the hydrated weight in air and submerged weight:

$\rho_{\text {bone }}=\rho_{\text {fluid }}\left(W_{\text {hydrated }} /\left(W_{\text {hydrated }}-W_{\text {submerged }}\right)\right)$

$\mathrm{W}_{\text {hydrated }}$ is the hydrated weight, $\mathrm{W}_{\text {submerged }}$ is the submerged weight and $\rho_{\text {fluid }}$ is the density of the fluid in which the specimen is submerged. Bone volume $\left(\mathrm{V}_{\text {bone }}\right)$ is calculated from the density of bone and its hydrated weight:

$\mathrm{V}_{\text {bone }}=\mathrm{W}_{\text {hydrated }} / \rho_{\text {bone }}=\left(\mathrm{W}_{\text {hydrated }}-\mathrm{W}_{\text {submerged }}\right) / \rho_{\text {fluid }}$

Then, Archimedes-based volume fraction $\left(\mathrm{VF}_{\mathrm{A}}\right)$ can be calculated from the bone volume and total specimen volume $\left(\mathrm{V}_{\text {totalspecimen }}=\right.$ external dimensions of the specimen):

$\mathrm{VF}_{\mathrm{A}}=\mathrm{V}_{\text {bone }} / \mathrm{V}_{\text {totalspecimen }}$

In applying this method of density measurement, it is important that the apparent density is measured because it is generally the most important factor affecting material properties such as yield strength and elastic modulus (Ashby, 1983). 
Apparent density is referred to as the mineralized tissue mass per total volume of the specimen, and is a function of the amount of bone (hydrated weight) present. Therefore, it is important to remove completely the intertrabecular fat (marrow) which could retain water and could affect the measurement of the hydrated weight. The Archimedes-based volume fraction has been performed using several marrow removal techniques before measuring the hydrated weight: (1) marrow removal using a high pressure stream of tap water (Arnold, 1960; Galante et al., 1970) and (2) marrow removal using alcohol (Carter \& Hayes, 1977). Since water and air jets seem unlikely to remove all the fat/marrow and alcohol is not a highly effective fat solvent, a new and effective method of fat removal using trichloroethylene and water together with centrifugation before measuring the hydrated weight of the specimen was developed (Sharp et al., 1990). The Archimedes-based volume fraction remains an effective, straight forward and simple technique for validating alternative methods of measuring volume fraction of trabecular bone (Ding et al., 1999; Beck et al., 1997).

6. Serial sectioning-based volume fraction, trabecular thickness, trabecular spacing and trabecular number

Trabecular morphometry has traditionally been assessed by studying two-dimensional (2D) histological sections. An improved method of histomorphometry has been developed for three-dimensional analysis using automated serial sectioning. Serial sectioning using a computer numerically controlled milling machine (serial milling) is an alternative method for producing three-dimensional image data that requires minimal post processing and is relatively simple and inexpensive compared to existing techniques using high resolution scans, i.e. micro-CT or micro-MRI (Beck et al., 1997). This 
method was further used in a comparison study of histological sections to micro-CT and magnetic resonance (MR) images (Müller et al., 1998; Kothari et al., 1998). The serial milling technique consists of four phases: specimen preparation; automated $\mathrm{CNC}$ milling to remove a prescribed thickness of the block of material; image capture at each level; and image processing (Beck et al., 1997).

\section{Mechanical Test}

Bone physiologic loading is primarily compression, followed by bending and then tension and shear. The traditional method of bone quality measurement is mechanical testing. There are a few biomechanical parameters which can be used to characterize the quality of bone. The main relationship is between load applied to a structure and displacement in response to the load (Turner and Burr, 2001). The slope of the elastic (linear) region of the load-displacement curve represents the stiffness of the bone structure. Traditional mechanical testing for bone include tensile tests, three or four point bending tests and compression tests (Figure 3). Bending tests are useful for measuring the mechanical properties of long bones. In the bending test the whole long bone is loaded in three or four-point bending, sometimes until failure. Tensile testing can be one of the most accurate methods for measuring bone properties when force is applied without inducing a couple bending moment (Turner \& Burr, 2001). However, tensile test specimens must be relatively large often between 15 to $25 \mathrm{~mm}$. This limits the usefulness of tensile testing for bone, particularly when testing trabecular bone because it is difficult to obtain such a large quantity of trabecular bone in animal samples such as guinea pigs, rats and rabbits. 


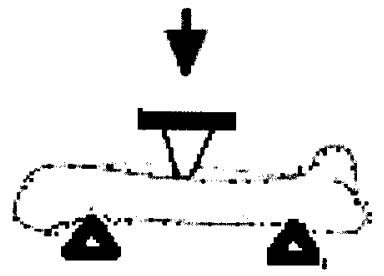

three point bending

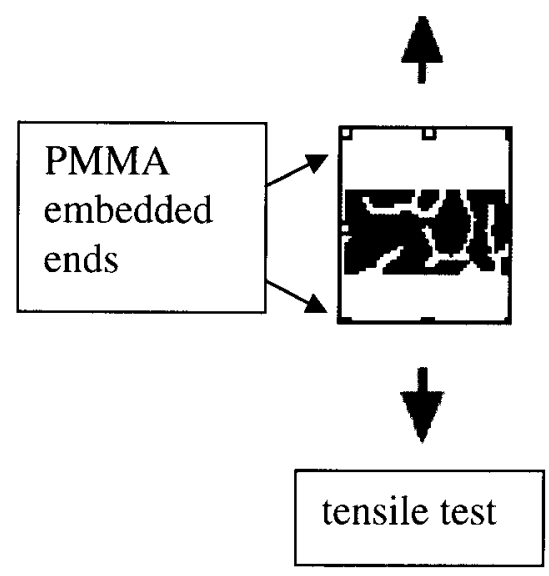

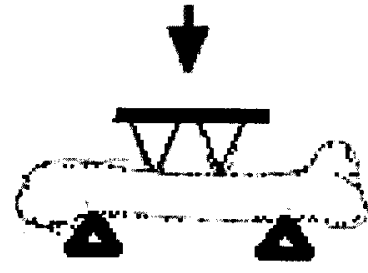

four point bending

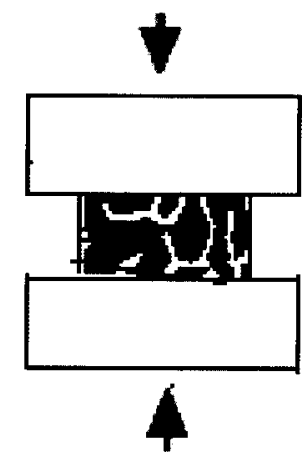

compressive test

Figure 3. Tensile (left), bending (center) and compression (right) tests.

On the other hand, compressive testing of bone specimens allows the use of relatively small specimens. Compressive testing using two rigid platens has been one of the best methods of mechanical testing to validate the micro-FE analysis and this has been widely used (Bourne et al., 2004; Orr et al., 2001; Wachter et al., 2001; Ding et al., 2003; Goulet et al., 1994). However, compressive tests using platens tend to be less accurate than tensile test due to end effects imposed on the specimen during testing. These end effects can be due to: 1) misalignment of the faces of bone specimens upon the platens (Figure 4);2) friction between the specimens and compression platens; 3 ) effects of machine compliance and unparallel surfaces of the bone specimen due to machining 
with an irrigated saw or burrs (Keaveny et al., 1993; Linde et al., 1989; Zhu et al., 1994; Brown and Ferguson 1980; Odgaard and Linde 1991).

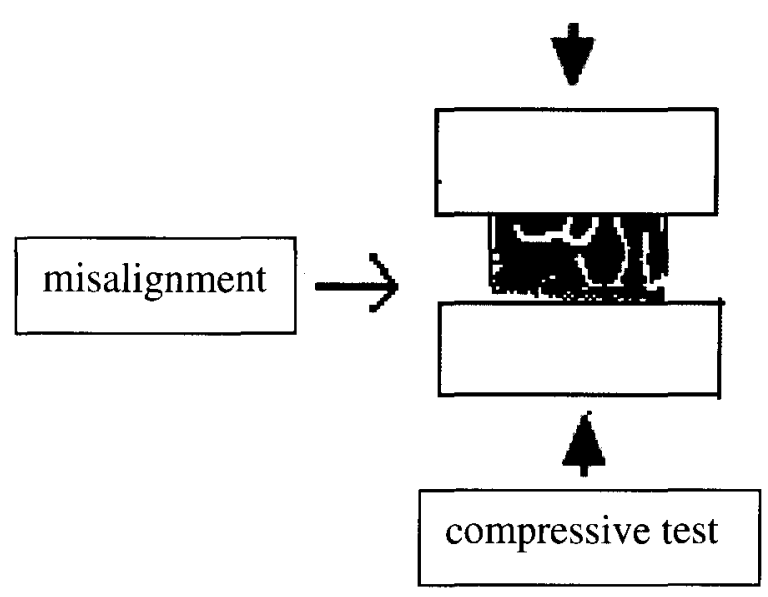

Figure 4. Misalignment of the faces of bone specimens upon the platens.

Misalignment of the faces of bone specimens upon the platens can be reduced with the placement of a pivoting platen in the load train (Figure 5) (Turner and Burr, 2001). Lubrication of the platens with mineral oil has shown to reduce friction (Linde and Hvid 1989). The precision of compressive test can be improved by making parallel cuts of the bone specimen using a diamond wafering saw (Turner and Burr, 2001). In addition, the accuracy of Young's modulus calculations could be improved by measurement of strain on the platens using an extensometer, a device that measures the deformation of a material to which it is attached, as this would eliminate the effects of machine compliance (Keaveny et al. 1997). 


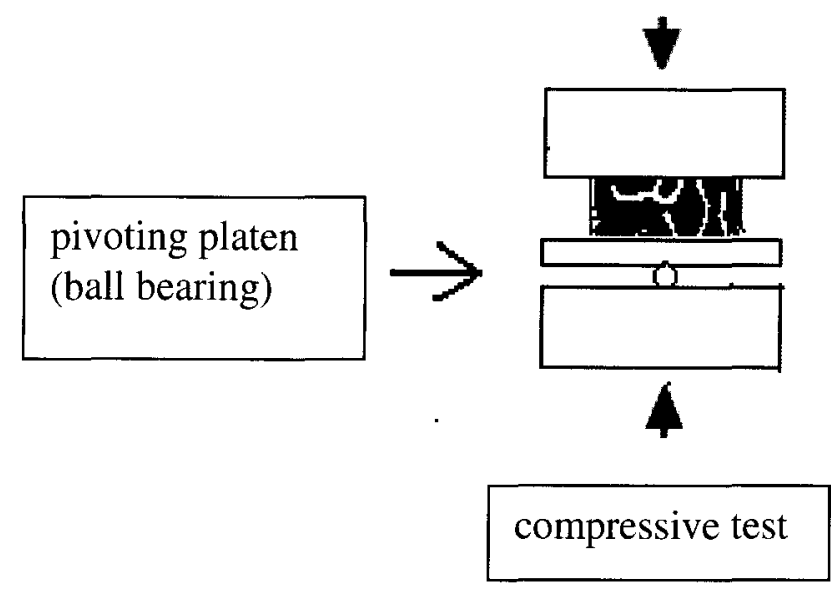

Figure 5. Placement of a pivoting platen (ball bearing) to reduce the misalignment. 


\section{METHODS AND MATERIALS}

The scope of the study includes in vivo and in vitro micro-CT scanning and image segmentation, direct conversion of micro-CT image to FE models, and experimental methods of density measurement, serial sectioning and compressive testing to validate the nondestructive techniques. Statistical analysis was performed on volume fraction, trabecular thickness, trabecular spacing and trabecular number based on micro CT and apparent stiffness based on micro-FE in comparison to experimental methods.

\section{Animal Model}

New Zealand white rabbits were chosen as the animal model in this study because the rabbit distal femur provides an adequate volume of trabecular bone with minimum measurement of $3 \mathrm{~mm}$ in each direction per condyle for the analysis. Rabbits have been established as the animal model for orthopedic research (Newman et al., 1995; Orr et al., 2001) and for measuring trabecular architecture using 3-D imaging techniques (Morita et al., 1994; Takahashi et al., 2002). A total of six six-month-old New Zealand white rabbits weighing approximately 4 to $5 \mathrm{~kg}$ were utilized in this study. Three rabbits were scanned twice in vivo seven days apart and three rabbits were only scanned once in vivo. All rabbits were then sacrificed and the femurs were scanned again in vitro. The size of the rabbit distal femur and its position of scanning made it possible to obtain $28 \mu \mathrm{m}$ and $14 \mu \mathrm{m}$ (nominal resolution) micro-CT scans in vivo and in vitro, respectively. All 
animals were cared for in compliance with a protocol approved by the university institutional animal care and use committee.

\section{Micro-CT Scanning}

\section{a. Scanning Hardware Description}

Micro-CT scanning was performed using a custom-made micro-CT scanner (Model ACTIS 200/225 Ffi-HR CT/DR system, BIR Inc., Chicago, IL with built-in X-ray system FXE 225.20, Fein Focus U.S.A.). It consisted of a specimen manipulator, X-ray source and detector (Figure 6). The machine is equipped with a $225 \mathrm{KV}$ x-ray source with a focal spot size of $5 \mu \mathrm{m}$. In addition, an anesthesia machine (Landmark Model VSA-2100, Hallowell Model 2000) was added to the scanner such that in vivo scanning could be performed with the animals sedated to minimize unwanted motion (Figure 6). A rabbit holder was custom made for the in vivo scanning procedure (Figure 7). In addition, a tube-like bone holder was custom made for the in vitro scanning procedure (Figure 7). The material used in making the rabbit holders was acrylic as it is optically transparent and transparent to $\mathrm{x}$-rays, relatively rigid, inexpensive and easy-to-use. 


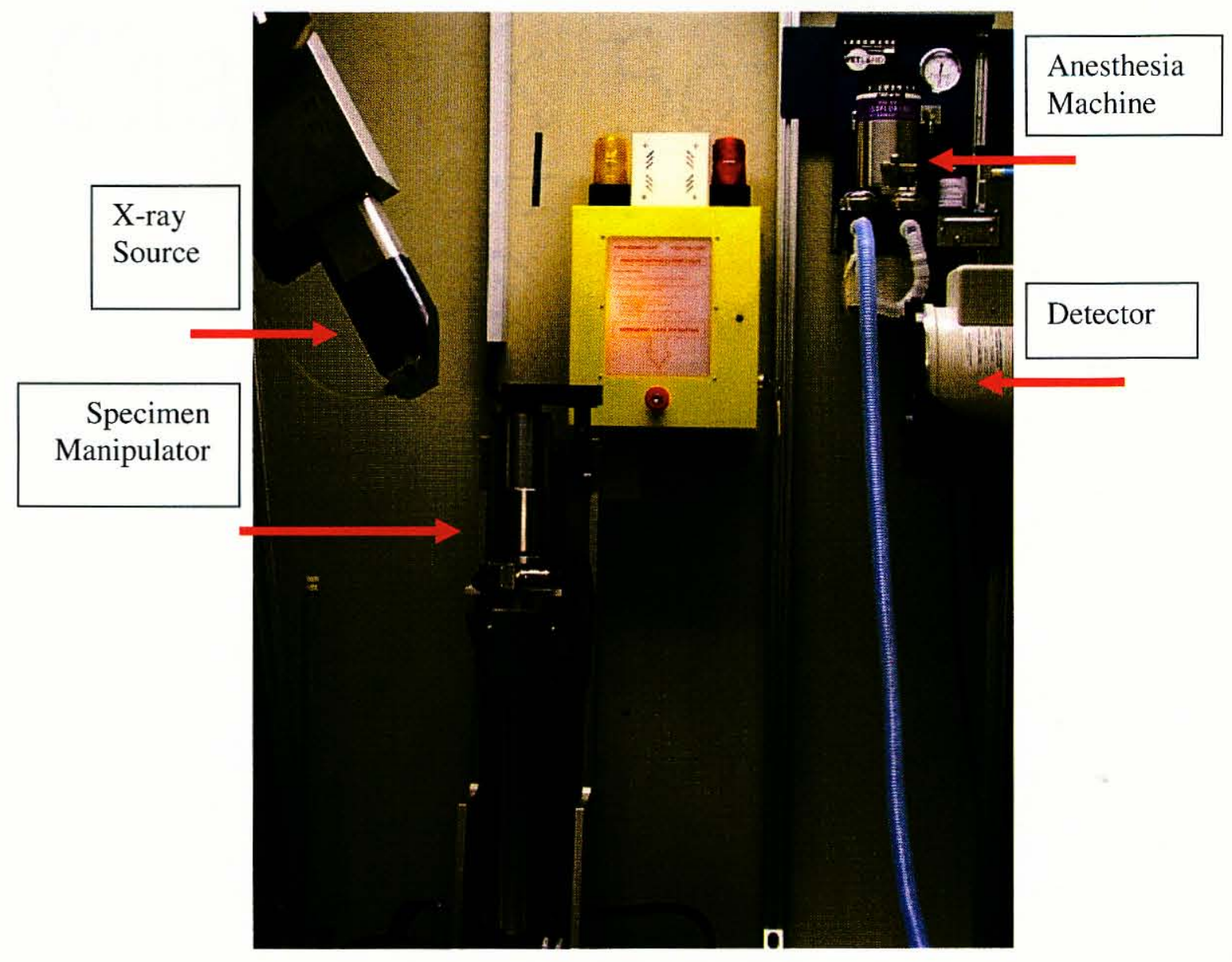

Figure 6. Micro CT Scanner
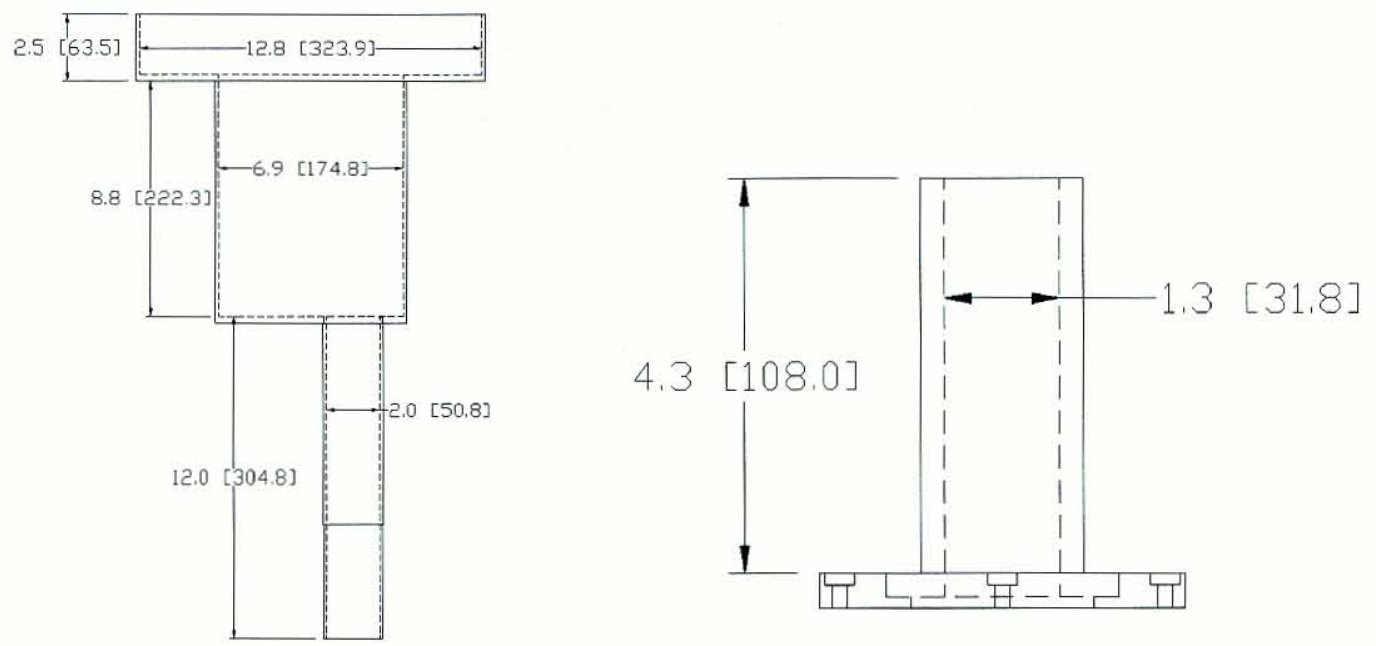

Figure 7. Rabbit Holder for in vivo scanning (left) and bone specimen holder for in vitro scanning (right) 
b. In Vivo Micro CT Scanning Procedure for a Rabbit Distal Femur

For the in vivo scanning, the rabbit was sedated with a cone mask under anesthesia at $1 \mathrm{~L} / \mathrm{min} \mathrm{O}_{2}, 14.7$ Psia $(1.0 \mathrm{Bar})$ and $70^{\circ} \mathrm{F}\left(21^{\circ} \mathrm{C}\right)$ with $3 \%$ isoflurane by volume. The cone mask was equipped with a two-way tubing (inhale and exhale) (Figure 8). The mask was secured around the head with a Velcro® strap. The rabbit was completely sedated within 10 to 15 minutes. A Velcro® strap was secured around the rabbit's ankle to identify and control the leg to be scanned. The rabbit was placed in the holder resting its front legs against the top column of the rabbit holder (Figure 9). The right hind leg was resting in flexion and the left hind leg was extended into the third column and held in place with the Velcro® s̊trap.
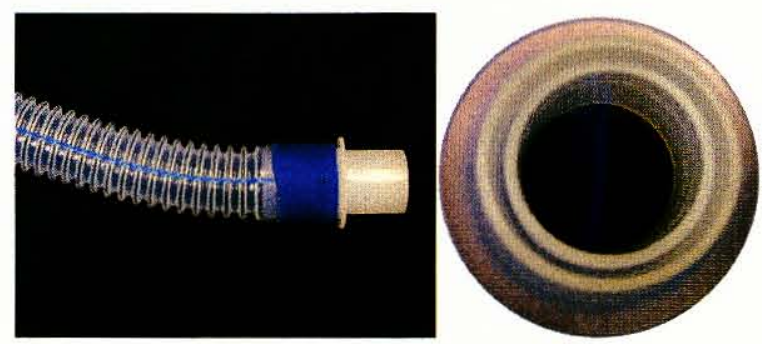

Figure 8. Two way tubing

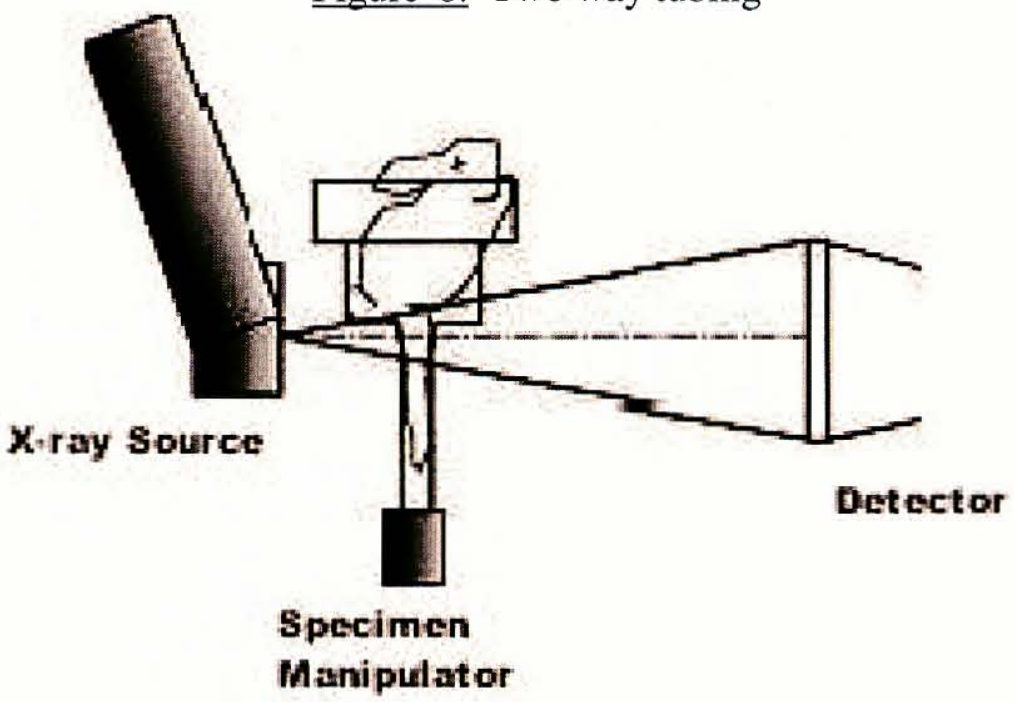

Figure 9. A Rabbit at a 60 deg angle in the holder 
Fabric padding was placed around its head and neck to provide anatomical support in its seating position (Figure 10). The rabbit holder was then placed on the specimen manipulator in the micro-CT scanner (Figure 11). The specimen manipulator was rotated around the z-axis and raised or lowered along the z-axis. Manual adjustment of the area to be scanned was made using the scanner software until the distal femur (region of interest) was in position for optimal scanning.

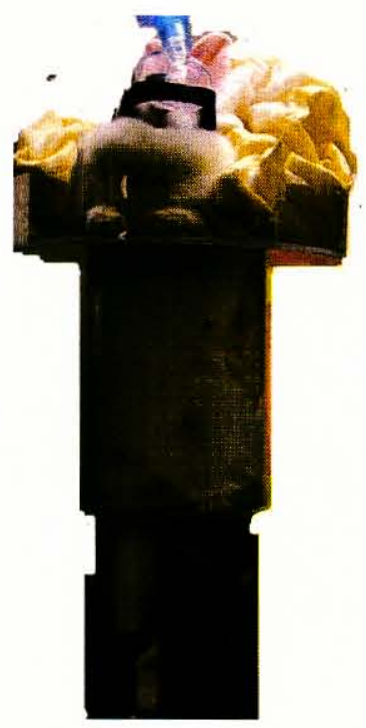

Figure 10. Rabbit's position

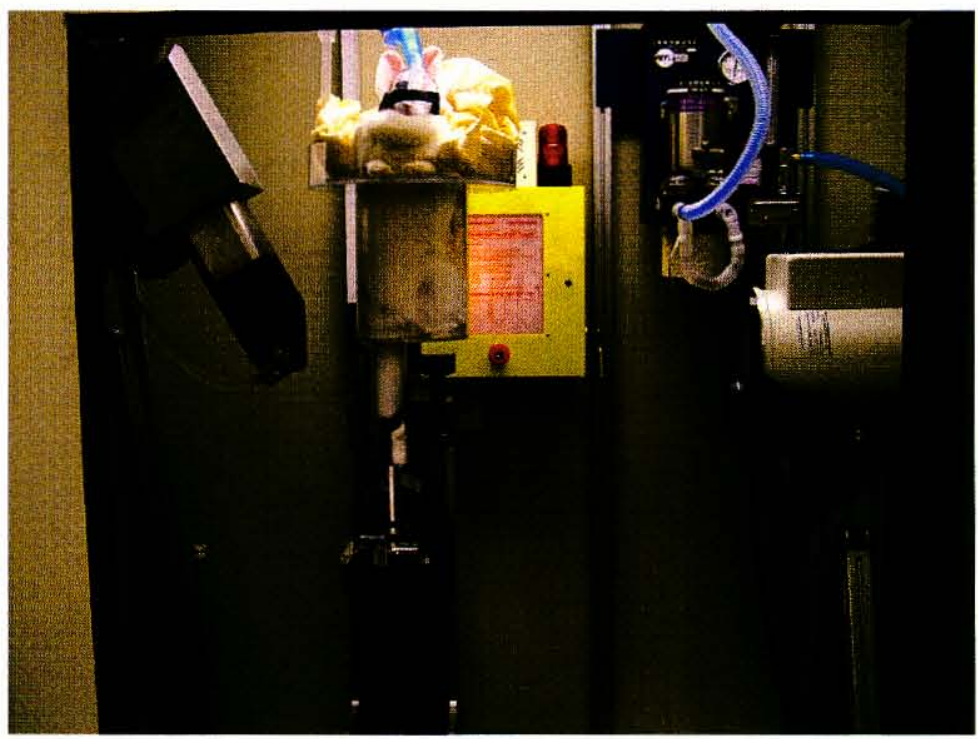

Figure 11. Rabbit holder in micro CT scanner 
A complete in vivo scan required three complete rotations (107 slices per rotation -4 min per rotation) at $28 \mu \mathrm{m}$ giving a total length of approximately $9 \mathrm{~mm}$ and took 12 min. $28 \mu \mathrm{m}$ is the best nominal resolution obtained in scanning a live rabbit distal femur in this arrangement based on the diameter of the tube at $50.8 \mathrm{~mm}$ using an offset scanning mode. The total time that a rabbit stayed in the micro-CT scanner was about 45 minutes for each leg scanned. After the scan, it took 5 to 10 minutes for the rabbit to wake up. An immediate reconstruction of the raw scanned files was performed to check for high resolution quality images. After this was confirmed, the rabbit was placed in its cage and either returned to its pen or euthanized with syringe injection of $1.5 \mathrm{ml}$ Beuthanasia-D (active ingredients: pentobarbital sodium and phenytoin sodium) in the necropsy room of the animal care facility.

\section{c. In Vitro Micro CT Scanning Procedure for a Rabbit Distal Femur}

After the rabbits were euthanized, the femurs were immediately harvested and kept frozen at $-20^{\circ} \mathrm{C}$ to make sure the trabecular architecture remained intact for all in vitro scanning and testing experiments (Linde and Sorensen, 1993). The rabbit femur was thawed and placed in a specimen holder of $25.4 \mathrm{~mm}$ in diameter and $152.4 \mathrm{~mm}$ in length (Figure 7). The bone was kept moist with saline soaked gauze. The in vitro scan was performed at $14 \mu \mathrm{m}$ nominal resolution based on the diameter of the $25.4 \mathrm{~mm}$ tube. Scans took approximately 2.5 hours and required 38 rotations ( 17 slices per rotation -4 min per rotation) at $14 \mu \mathrm{m}$ resulting in a total scan length of approximately $9 \mathrm{~mm}$. This was accomplished by volumetric scanning where the specimen manipulator rotated $360^{\circ}$ and moved up to the next level for the next rotation. 


\section{Micro-CT Image Processing and Analysis}

a. Image Preparation

The in vivo scans consisted of 321 slices while the in vitro scans consisted of 663 slices spanning a $9 \mathrm{~mm}$ distance from above the condylar notch to the bottom of the medial condyle (volume of interest). This was to insure an approximately $64 \mathrm{~mm}^{3}$ volume of interest was completely contained within the vertical distance of $9 \mathrm{~mm}$ scan, which was used for both image and finite element analysis. Individual slices were reconstructed as $8 \mathrm{MB}$ tiff image files (Figure 12). Since all bone images were saved in tiff format, they could be read in Matlab 7.0 (The Mathworks, Inc.), ImageJ 1.34s (National Institute of Health, USA) and Volume Graphics StudioMax 1.2.1 (Volume Graphics GmbH, Germany). These files were large files and there was a limitation in image processing as well as finite element analysis due to limited computer processor, memory and hard disk space available. Therefore, it was necessary to crop the slice images, thus shrinking the image size to approximately 2.5MB (Figure 13) in ImageJ or Matlab(Program A in Appendix I) to efficiently perform image processing and finite element analysis. The images were then rotated in $\mathrm{x}$ and $\mathrm{y}$-direction (Figure 14) using the rotate function available in Matlab (Program B in Appendix I) and cropped again (Figure 15) so that they could be realigned or registered as a 3-D stack. This was to insure that the in vivo scan at time day-one (T1) was three dimensionally realigned to the in vivo scan at time day-seven (T7) such that the same volume of interest from the same rabbit but scanned at different times could be compared and analyzed. 

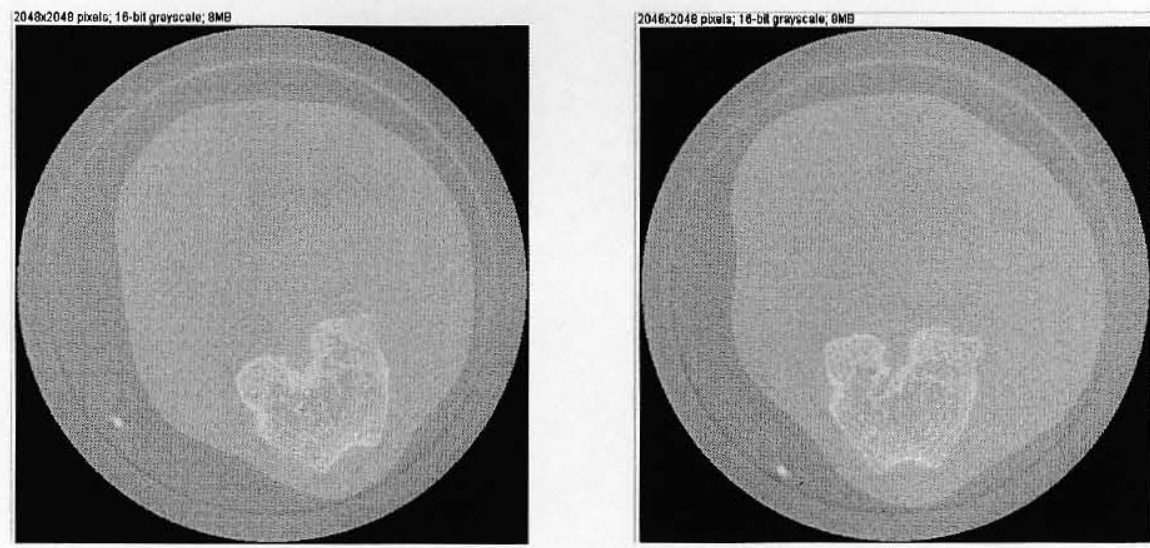

Figure 12. Tiff images $(8 \mathrm{MB})$ for the same rabbit scanned at different times
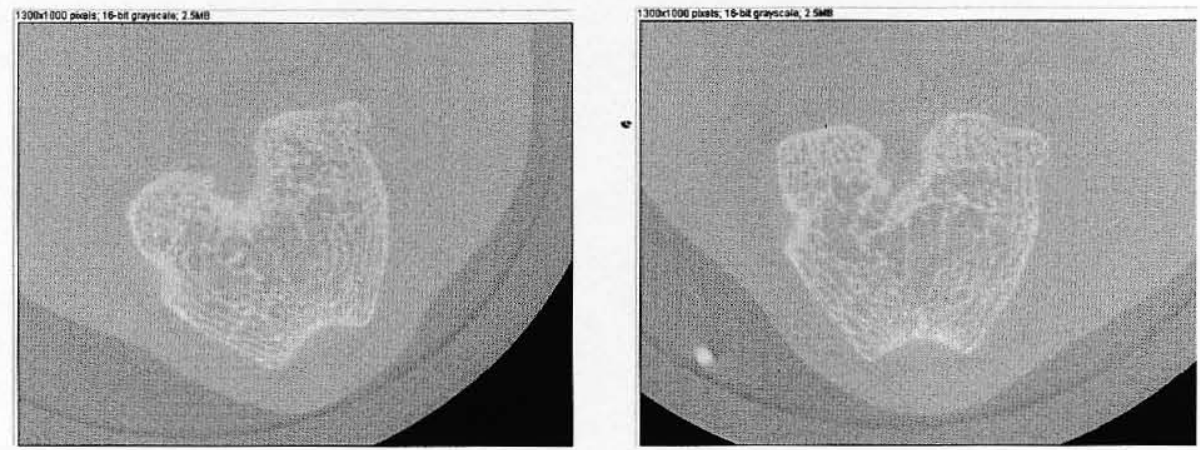

Figure 13. Cropped 2.5 MB tiff images for the same rabbit scanned at different times
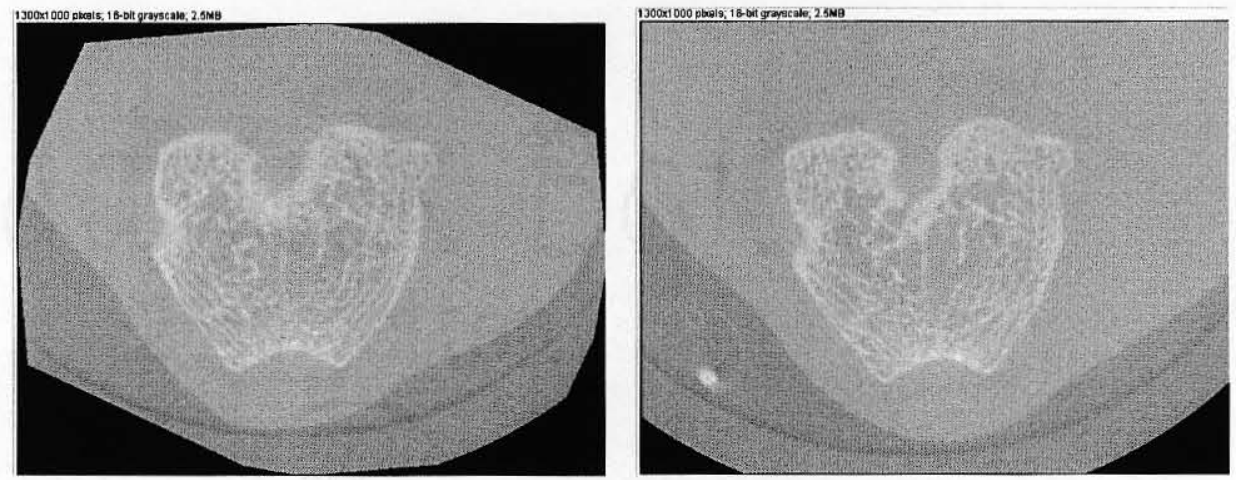

Figure 14. Image (left) rotated to match Image (right) 

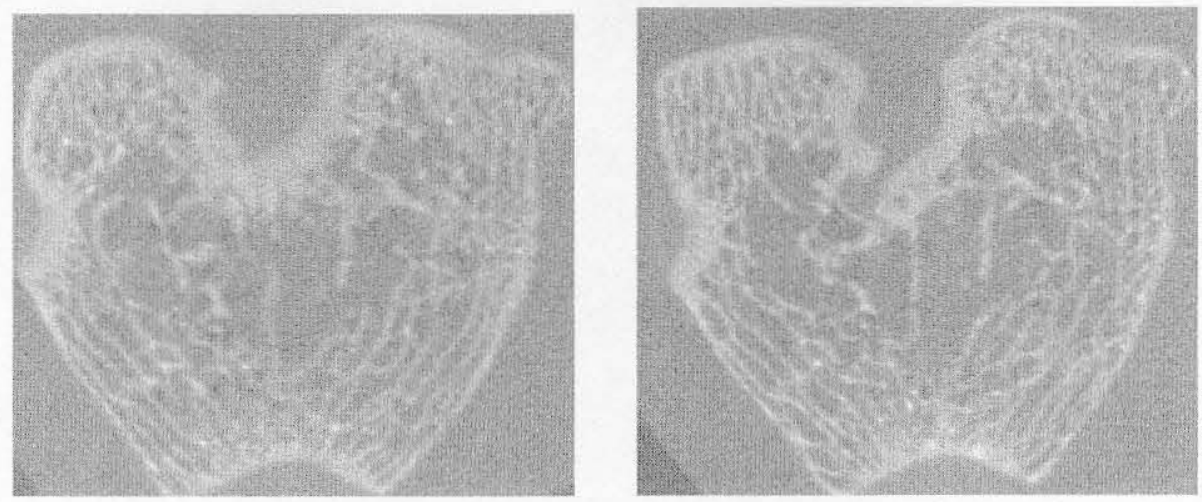

Figure 15. Cropped Images to be realigned in 3-D stacks

The registration method is a custom registration algorithm technique written in Matlab (Yang, et al. 2005). The image registration is a data matching system, which matches the $3 \mathrm{D}$ voxel information contairfed within an image stack to the $3 \mathrm{D}$ voxel information of the same bone scanned at a different time (Figure 16). The method is based on maximizing mutual information (Maes, et al. 1999). The registration method is limited to $30 \mathrm{deg}$ of rotation and translation and images of equal voxel sizes. Therefore, it is best to make all scans as close as possible in the $\mathrm{x}, \mathrm{y}$ and $\mathrm{z}$ axis using the similar level and amount of slices, similar region, approximately same size of image as well as the direction of the images. Since images of equal voxel size can only be three dimensionally aligned, the in vitro scans (Vit) taken at higher resolution $(14 \mu \mathrm{m})$ must be degraded to the same resolution $(28 \mu \mathrm{m})$ of the in vivo scans (T7) (Figure 17). 


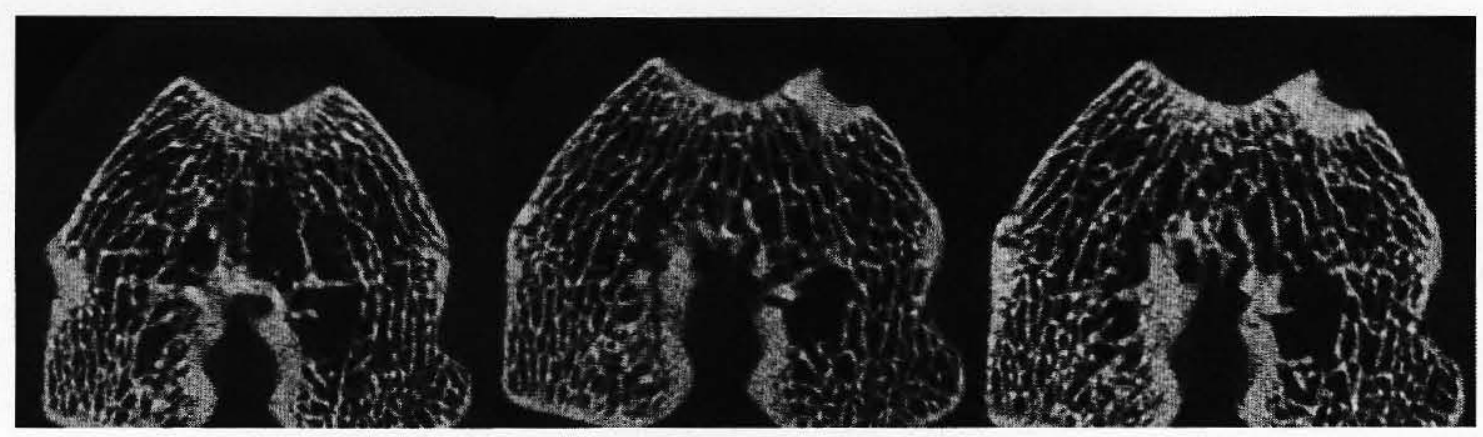

Figure 16. Micro CT images - before registration (right), registration reference image (center) and after registration (left)
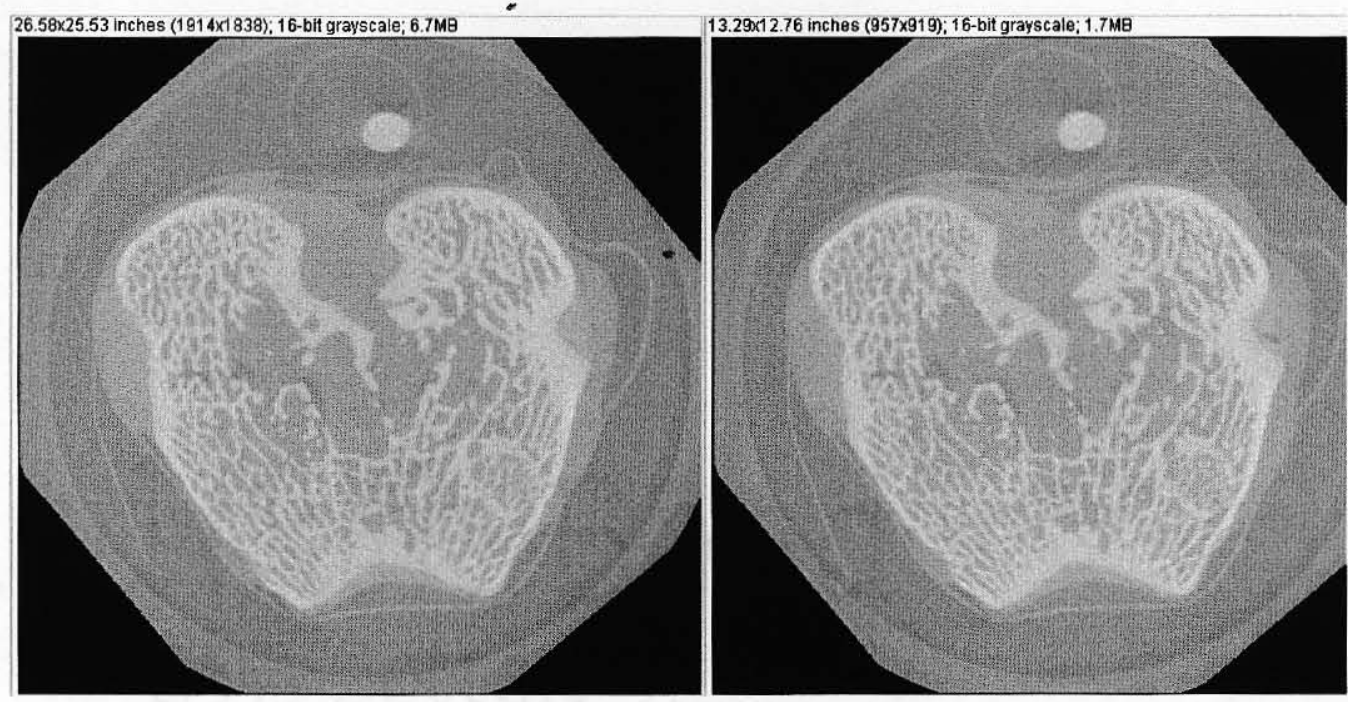

Figure 17. Images of $14 \mu \mathrm{m}$ (left) and degraded to $28 \mu \mathrm{m}$ (right)

b. Thresholding Technique and Segmentation Process

The segmentation process involves isolating the original bone image into bone tissue and non-bone tissue (marrow, soft tissue). The first step was to identify the region of interest which only contained trabecular structure on the bone image. It is best that this cubic region of interest has dimensions of a minimum of $3 \mathrm{~mm}$ in each direction ( $\mathrm{x}, \mathrm{y}$ and $\mathrm{z}$ ). When cropped, this leaves a volume of interest of a minimum of $27 \mathrm{~mm}^{3}$. The reason for having a minimum dimension of $3 \mathrm{~mm}$ in each direction is to insure that trabecular bone can be considered as a continuous structure (Harrigan, et al. 1988). The 
required maximum of a $3 \mathrm{~mm}$ slice in each direction is due to (1) anatomical location of the rabbit distal femur after image registration; and (2) computational limitation of maximum number of elements in a model for subsequent finite element analysis. The thresholding technique used in this segmentation involved slice-by-slice global thresholding. This global threshold was performed in MatLab using a function called "graythresh" which uses Otsu's method (Otsu, 1979). The "graythresh" function was slightly modified such that it could read 16 bit images rather than just the original "graythresh" function which only reads 8 bit images. This was because all the micro-CT images were 16 bit tiff images giving a range of gray level values of 1 to 65535 which represented the bone tissue density. The slice-by-slice global threshold obtained from bone image one was used to threshold the bone image one and this process continued until it reached the end of the image stack. The threshold value was based on an average value from a group of grayscale values that represented trabecular bone information (Figure 18). The thresholding technique in this segmentation was a custom written program in MatLab 7.0 (Program C in Appendix I). When all the images were segmented, they were converted to 8-bit files of black with a value of 0 (no bone) and white with a value of 255 (bone tissue). In addition to the slice-by-slice global threshold, a percentage correction factor was added to the threshold value for all the $28 \mu \mathrm{m}$ in vivo scans as they were overestimating the volume of bone compared to the $14 \mu \mathrm{m}$ in vitro scans. The percentage correction factor was calculated such that the volume fraction predicted from the in vivo scans was able to match the volume fraction from the in vitro scans. First, the volume fractions of in vivo and in vitro bone images were obtained. Then, correction factors of $0.5 \%$ and $1 \%$ were added to the threshold value for medial 
and lateral condyles respectively. The volume fraction of in vivo bone image was recalculated. Subsequently, a $0.02 \%$ correction factor was added, if the volume fraction of in vivo bone image was still higher than in vitro bone image, or subtracted, if the volume fraction of in vivo bone image was rather lower than in vitro bone image, to the previous correction factor for every difference of 0.001 in volume fractions of in vivo and in vitro bone images. This percentage correction factor was chosen by trial-and-error analysis.

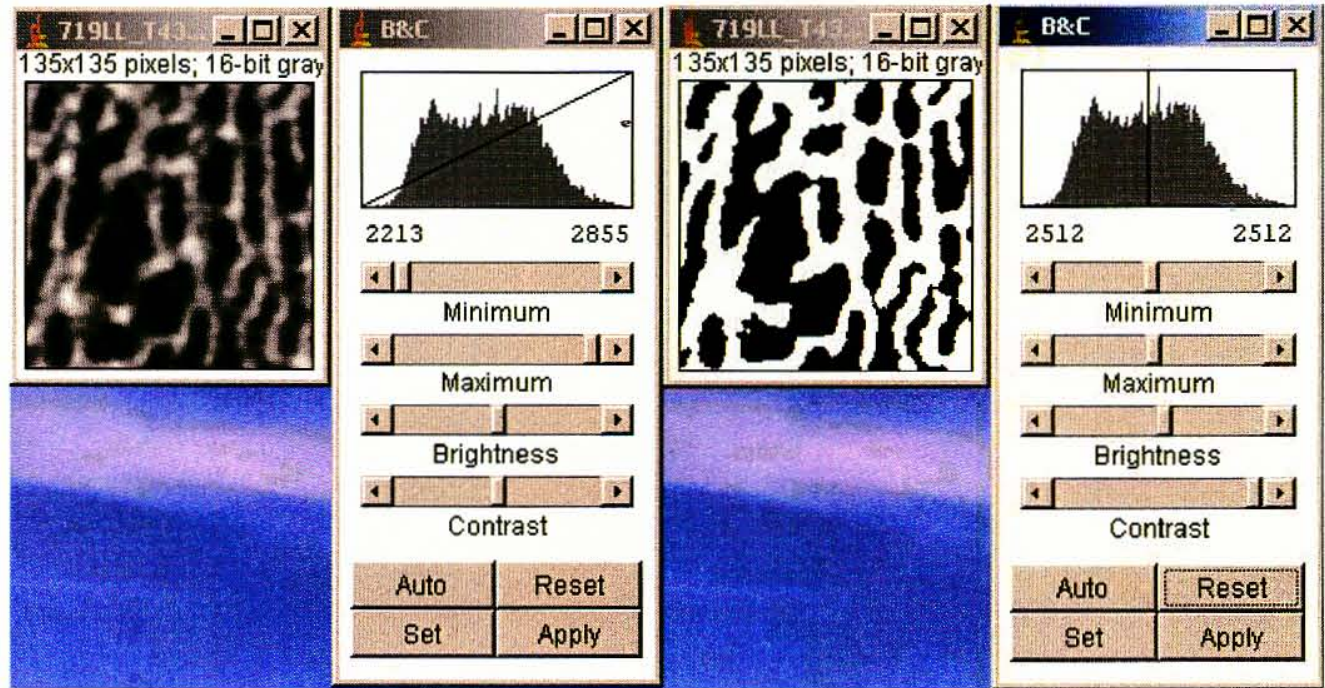

Figure 18. Slice-by-slice global thresholds

The volume fraction (VF), trabecular thickness ( $\mathrm{Tb} \mathrm{Th}$ ), trabecular separation (Tb $\mathrm{Sp}$ ) and trabecular number $(\mathrm{Tb} \mathrm{Nm})$ were measured and compared between the in vivo scans at two times; day-one (T1) and day-seven (T7). Then, the in vivo scans (T7') were compared to the higher resolution in vitro (Vit) scans. T7' is the same scan as T7 but the volume of interest used in $\mathrm{T} 7{ }^{\prime}$ is slightly different from $\mathrm{T} 7$ due to the three dimensional realignment of $\mathrm{T} 7$ to Vit. $\mathrm{VF}, \mathrm{Tb} \mathrm{Th}, \mathrm{Tb} \mathrm{Sp}$ and $\mathrm{Tb} \mathrm{Nm}$ were all measured using 
Volume Graphics Studio Max 1.2.1. If Volume Graphics Studio Max 1.2.1 is not available, VF can also be measured in a custom written MatLab program (Program D in Appendix I) which gives the same results as measured in Volume Graphics Studio Max

\subsection{1.}

\section{Bone Preparation Technique}

Due to the small size of rabbit bones and difficulties with cleaning cored samples, it was decided to use bone cubes rather than cylindrical bone cores. All the bone cubes were sized around $3 \mathrm{~mm}$ to $4 \mathrm{~mm}$ on each axis of $\mathrm{x}$ (width), $\mathrm{y}$ (length) and $\mathrm{z}$ (depth). The bone cubes were first prepared for Archimedes' experiment and then used for either serial milling or for mechanical testing (compression). It was necessary to follow a consistent cutting procedure to obtain useful bone specimens. First, a transverse cut was made at the level of the distal third of the rabbit femur leaving a small portion of the shaft with the condyles intact (Figure 19). Then, the condyles were separated through the condylar notch into the medial and lateral portions (Figure 20). Another cut was made on the proximal part of the condyle which was aligned parallel to the distal part (Figure 21). The proximal part was fixed to a stainless steel plate aligning the lateral or medial axis as vertical as possible (Figure 21). The stainless steel plate was attached to the diamond saw and subsequent cuts of anterior-posterior, medial/lateral-center and distal-proximal were made leaving a cubic bone sample of approximately $4 \times 4 \times 4 \mathrm{~mm}^{3}$ in volume. 

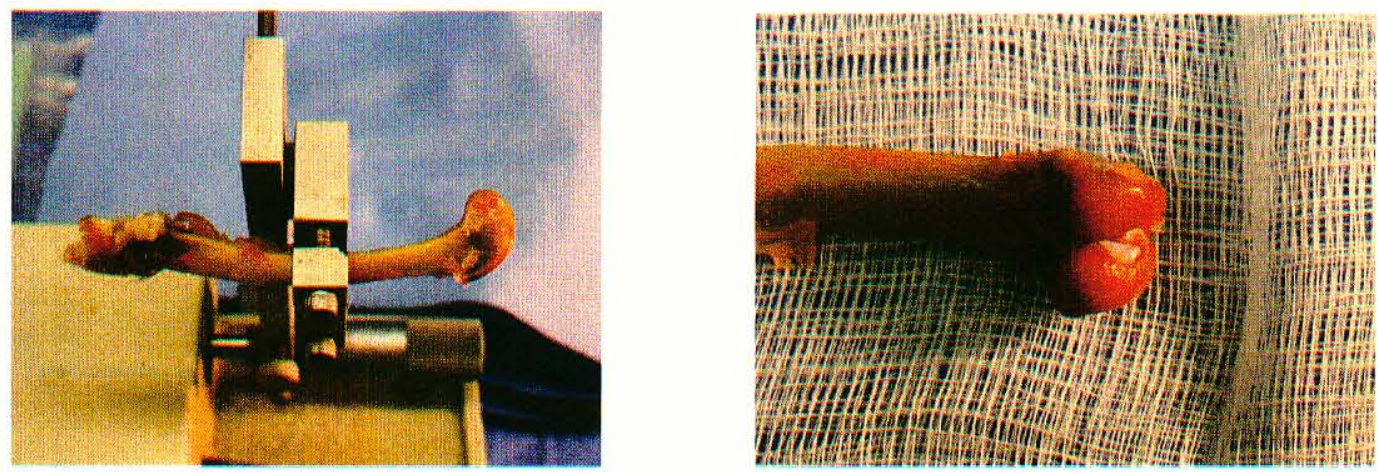

Figure 19. Transverse cut at the distal third of the rabbit femur.
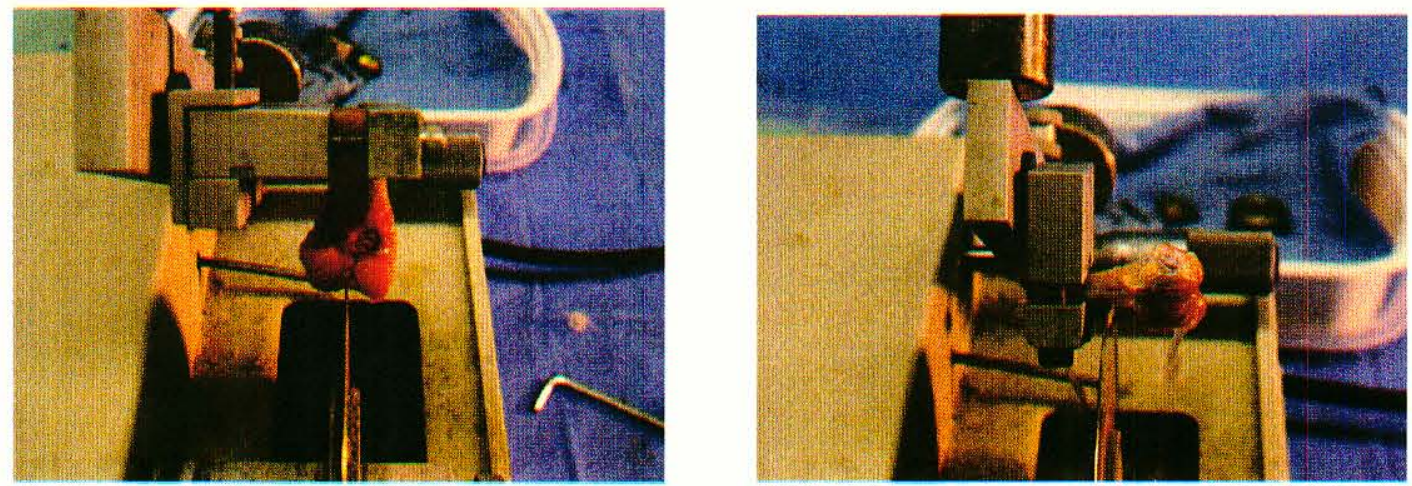

Figure 20. Separating the medial and lateral condyles.
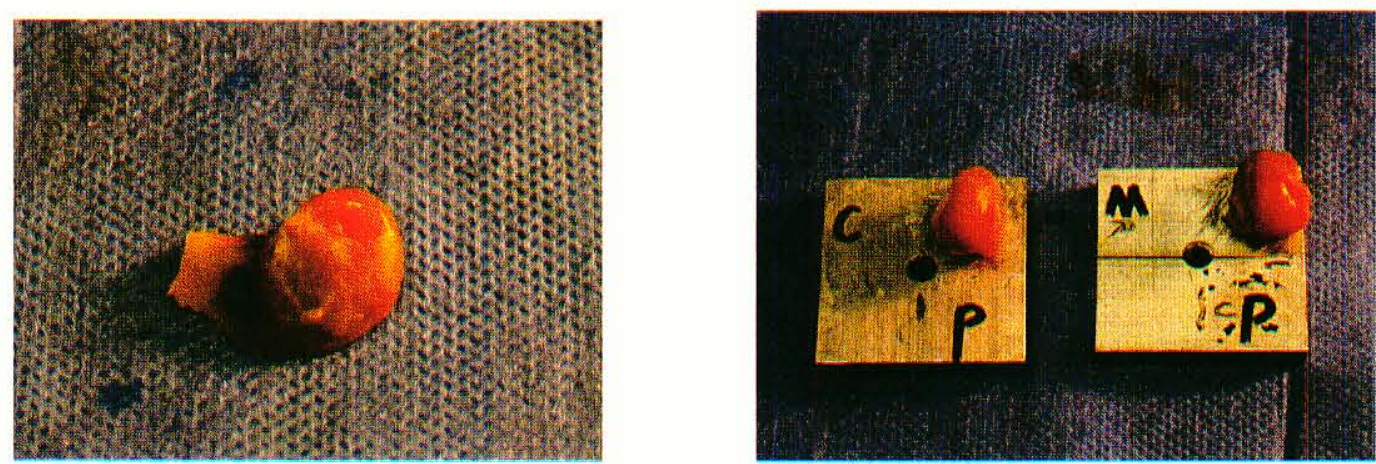

Figure 21. Proximal Condyles were fixed with adhesive on stainless steel plates.

A fine tip permanent marker was used to label posterior (violet), medial/lateral (red) and distal (green) on a bone cube (Figure 22). Using the low speed diamond saw, special cuts were made for Archimedes and serial milling experiments, since when the bone was cleaned; all marks were dissolved in the chemical solution. The posterior 
surface had a shallow horizontal line cut in the middle of the face while the distal surface had 2 shallow horizontal lines cut and the medial/lateral surface had a shallow diagonal line cut. These cuts were considered negligible because they accounted for $0.70 \%$ error in total volume fraction.

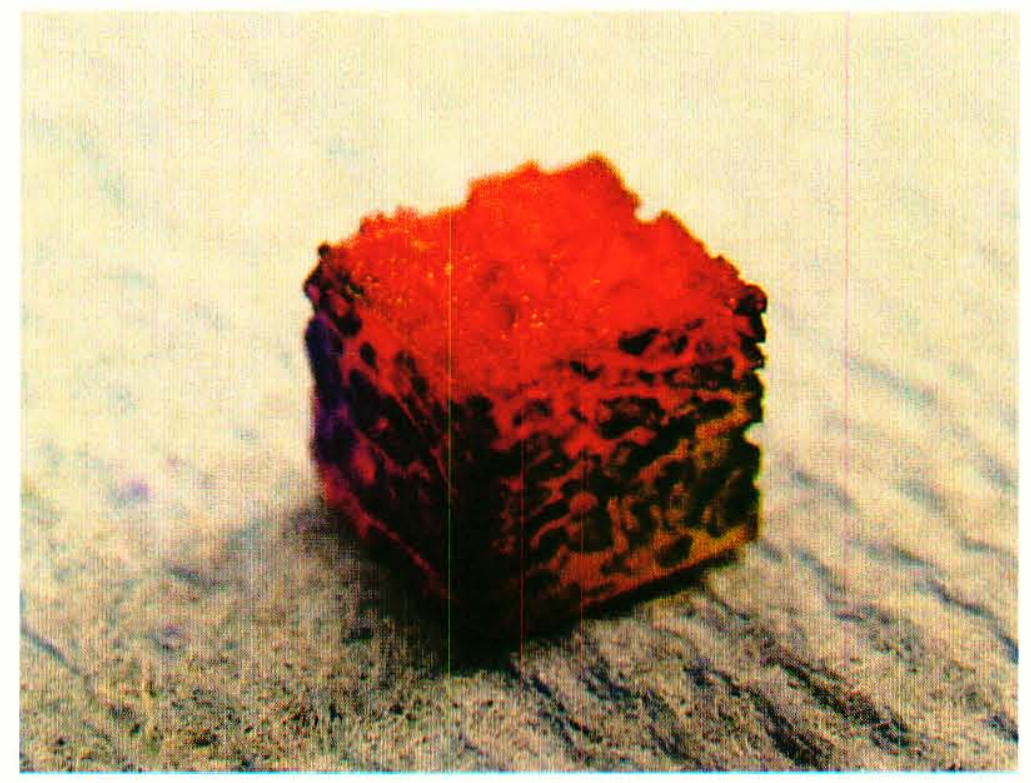

Figure 22. A bone cube with posterior (violet), medial/lateral (red) and distal (green)

4. Archimedes'-based density and volume fraction measurement

To confirm the validity of the segmentation technique on micro-CT images, it was necessary to perform density measurements using Archimedes' principle as it provided a very basic and easily measured volume fraction value. Archimedes' experiment required the following steps. The trabecular bone cubes were cleaned using tricholoroethylene in an ultrasonic agitation for one hour followed by rinsing with a waterpik system (model WP-60W) at a pressure control speed of $60 \mathrm{psi}$ using about $500 \mathrm{ml}$ of water on all sides of the cube (Figure 23). The cleaning process was repeated five times to ensure marrow removal without trabecular damage. 

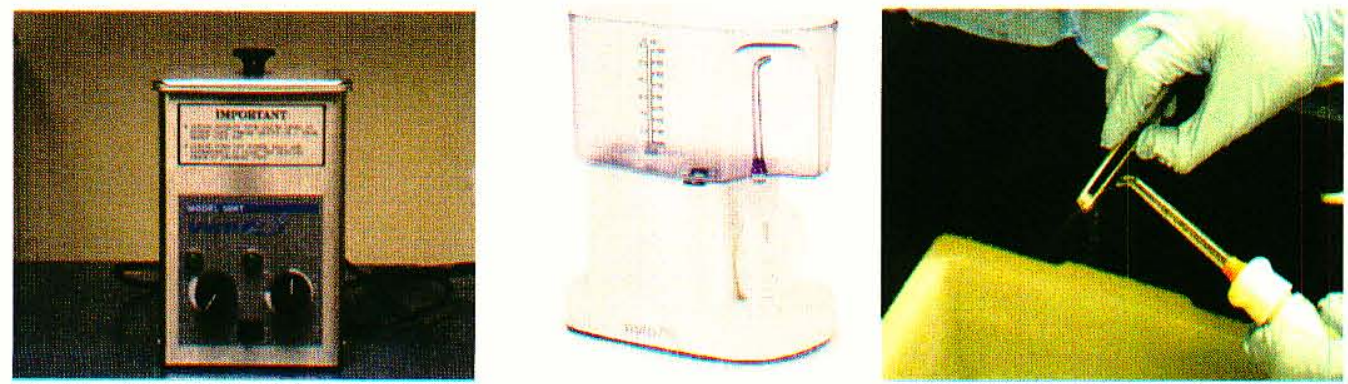

Figure 23. Ultrasonic bath (left), waterpik (middle) and rinsing procedure (right)

Rehydration of trabecular bone was performed in distilled water under vacuum for 4 hours. Every hour, the vacuum was released and reapplied. This was to insure the bone was evenly rehydrated. The position of the bone cube was adjusted each time to ensure an even vacuum was applied during rehydration. Any bubbles on the bone surface were observed to insure that they had been reduced at the end of the hour of rehydration. An analytical balance (model AG135 from Mettler Toledo, Inc.) was setup for all density measurements based on Archimedes' principle (Figure 24). First, the submerged weight was measured. Then, the bone cube was centrifuged for $3 \mathrm{~min}$ at $200 \mathrm{~g}$ with a small piece of dry gauze filling $3 / 4$ of the micro-centrifuge tube. The bone cube was left in air for 30 sec prior to measuring the hydrated weight. The bone cubes were scanned again at 14 $\mu \mathrm{m}$ nominal resolution after the density measurement to verify the accuracy of micro-CT imaging technique.
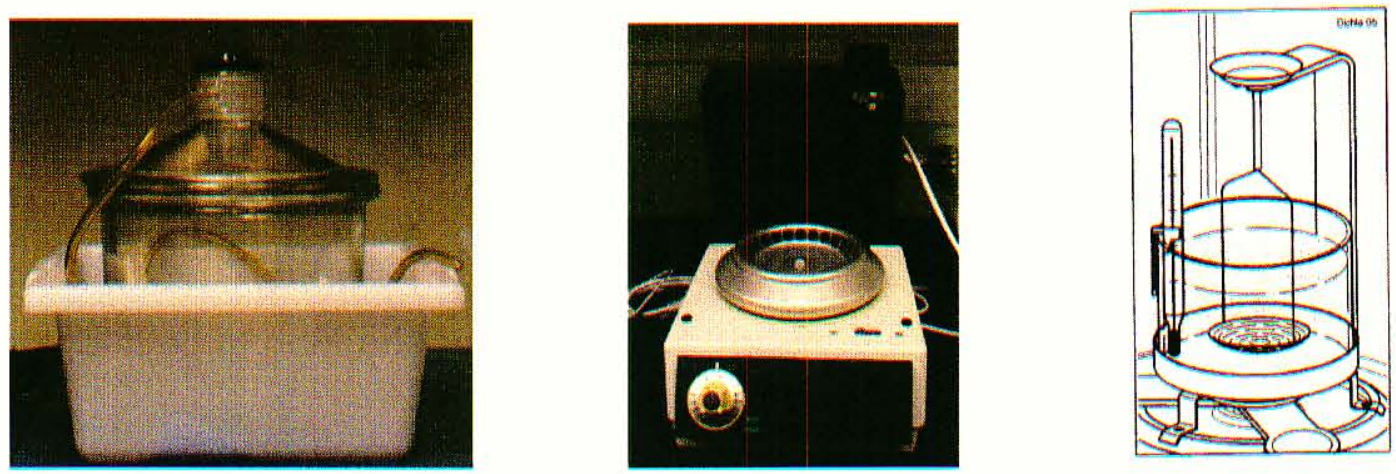

Figure 24. Vacuum (left), centrifuge-Microspin 24P (center), Analytical balance (right). 


\section{Serial Sectioning (In Vitro)}

Serial sectioning with high contrast imaging was performed to further validate the segmentation technique. Cleaned bone cubes were stained black at room temperature by immersion in a $10 \%$ (wt) silver nitrate $\left(\mathrm{AgNO}_{3}\right)$ solution, first for 30 minutes in an ultrasound chamber and then for 20 hours. This process was repeated twice with a fresh solution each time. The bone cubes were then rinsed in water using the waterpik system as previously explained, drained of water trapped in the trabecular pores using a centrifuge as previously explained, and placed into $100 \%$ ethanol where they were exposed to $365 \mathrm{~nm} /$ long-wave UV light using a portable/rechargeable lamp (model UVA26) for 120 minutes (Figure 25). For optimal exposure, glass vials were used, the specimens were placed on their side as close as possible, approximately $2 \mathrm{~cm}$, to the UV light source, and rotated from one side to another for all six sides of the bone cubes every 20 minutes. Therefore, all sides of the bone cubes had 20 minutes of exposure. Staining occurred by diffusion of the silver nitrate into the bone with substitution of $\mathrm{Ag}^{++}$ions for $\mathrm{Ca}^{++}$ions. The UV light reduced the Ag, giving the bone a deep black matted nonreflective color.

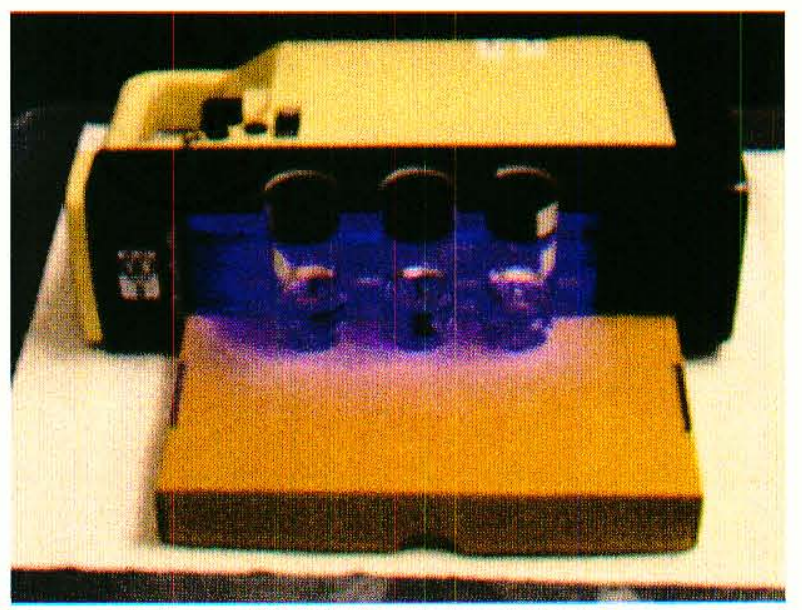

Figure 25. Bone cubes exposed to $365 \mathrm{~nm} /$ long-wave UV light 
Embedding was achieved as follows. First, bone cubes were dehydrated in fresh $100 \%$ ethanol for 20 hours at room temperature and this process was repeated three times in fresh ethanol. Meanwhile, a partially prepolymerized methylmethacrylate was prepared as the bone embedding media. Preparation of this media was crucial. The methods of preparation were followed from the technical data sheet from Electron Microscopy Sciences. However, for this particular bone embedding application, maintaining the trabecular bone architecture, was important; thus, the partially prepolymerized methylmethacrylate was prepared by mixing n-butyl and methyl methacrylate in the ratio of $3: 2(\mathrm{vol} / \mathrm{vol})$. In addition, a methacrylate catalyst solution was prepared simply with methyl-methacrylate monomer with benzoyl peroxide paste in the ratio of 9:1 (wt/wt). The embedding media was made up of the catalyst solution and the prepolymerized methylmethacrylate in a ratio of $1: 5(\mathrm{vol} / \mathrm{vol})$. Then, a $15 \%$ mixture by volume of white pigments (Tap epoxy pigmentation, Tap Plastics Corp., El Cerrito, CA) was added to the embedding media.

A specially designed bone mold with an inner diameter of $7 \mathrm{~mm}$ and outer diameter of $9 \mathrm{~mm}$ with a cylindrical $\mathrm{T}$ plug was made using polytetrafloroethylene (PTFE) for this specific application (Figure 26). PTFE can withstand heat, has low frictional coefficient, and is relatively inert. This was to insure proper embedding and that the embedded bone sample could be easily extracted. Bone cubes were placed into the embedding media in $10 \mathrm{ml}$ vials and vacuum was applied for 30 minutes. This process was repeated three times with fresh embedding media replacing the previous one. Meanwhile, the bone mold was covered with masking tape such that the upper ends of the tape extended to approximately twice the length of the bone mold (Figure 26). 

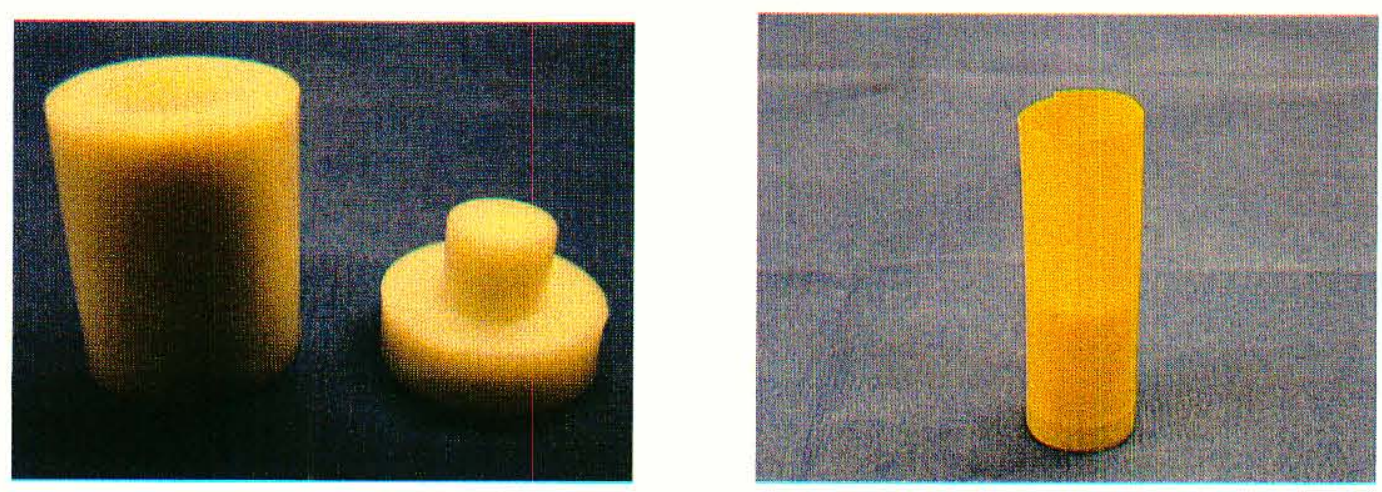

Figure 26. Cylindrical bone column (left) and bone mold covered with masking tape (right)

Then, the bone cubes were transferred into the bone mold with the distal face at the bottom of the mold just as in the scanning procedure of the bone cubes. The embedding media was poured into the mold covering 3 times the depth of the bone cubes, which not only covered the inner mold but also some of the spaces within the masking tape. The molds were placed in $20 \mathrm{ml}$ glass vials and degassed in the vacuum chamber for 30 minutes. This degassing process was repeated 3 times. On the last cycle, the vials were tightly capped, placed into a water bath, and cured in an oven at $65^{\circ} \mathrm{C}$ for 24 hours. Care was taken to avoid air bubbles trapped within the embedding media which could cause error in image processing. In order to control the rate of the polymerization reaction, the reaction was slowed by surrounding the curing specimen with a water bath. The result is a high contrast black and white bone-polymer composite. The composite was left for 12 hours to cool. Then, it was taken out from the bone column leaving the whole composite as a cylindrical bone cast. The excess material of the cylindrical bone cast was removed using the low speed diamond saw leaving just the cylindrical specimen ready for serial milling (Figure 27). 


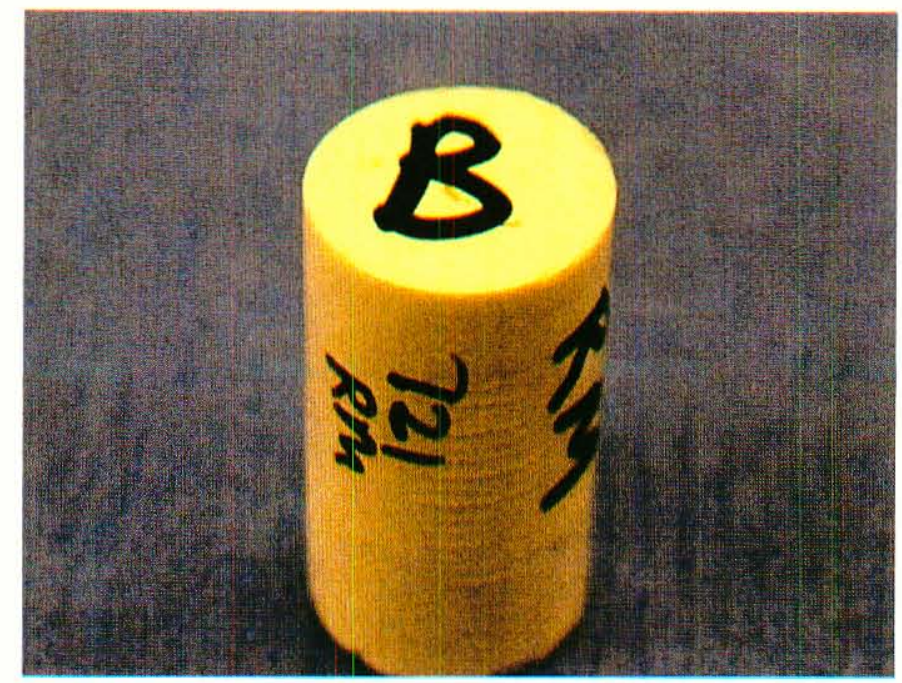

Figure 27. A cylindrical bone cast.

The serial milling was performed on a custom-made micromilling machine (Figure 28). The main hardware component is the Ultra-High-Precision Micro Milling/Drilling Machine (micromill) with a computer-numeric-controlled (CNC) milling station, which is quite similar to those at the macro-scale. It was exclusively developed by Craig Friedrich (Michigan Tech) in collaboration with Dover Instruments, now Danaher Motion (Westboro, MA). Its vertical axis is mounted with a digital camera (CCD Model CCD-1300-Y/HS - Roper Scientific, USA) with 1300 by 1030 overall pixel resolution and $6 \mathrm{X}$ max magnification with a ring illuminator (Standard fiber optic ring illuminator). The digital camera was connected to a personal computer (PC) via PCI card interface (MicroMax 5MHz Controller connects camera to PC) which produced 8-bit, $750 \times 750$ pixel gray-scale tiff images. 

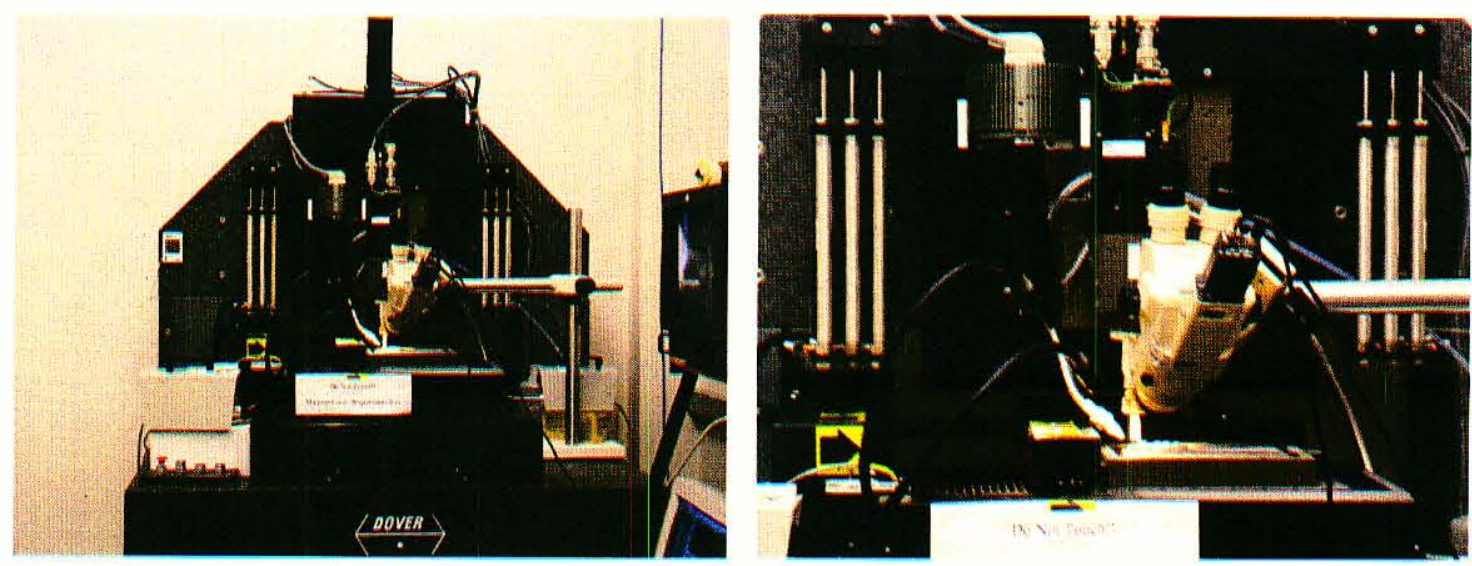

Figure 28. Micromill (left) and close up view of micromill (right).

The imaging process itself consisted of three steps. First, each specimen was mounted on a countersunk surface on a circular plate made of 6061-T6 aluminum (Figure 29). This circular plate was mounted on the mill table using three hex-headed screws; first screw attached at the center of the circular plate and the other two screws were secured at the two opposite ends of the circular plate. The cylindrical specimen was oriented with its central axis parallel to the vertical axis of the milling machine. Second, the milling machine was programmed to remove a thin layer (typically $14 \mu \mathrm{m}$ ) per pass from the specimen using a $3 / 8$ in. carbide-impregnated, two-fluted end mill (Bassett USA, 52032MSE, Pacific Tool, Oakland, CA) cutting at a spindle-rate of $3000 \mathrm{rpm}$ with a specimen feed rate of $1-1.5 \mathrm{~mm} / \mathrm{sec}$. This tool was replaced after every three specimens due to blunting of the tool which roughened the machined surface and degraded the image quality. While milling, an air blower was used to remove the debris as they were cut from the specimen since residual debris could obscure the images and increase the roughness of the machined surface; thereby, producing a smooth, dust- and chip- free surface for imaging (figure 30). Finally, the specimen was moved into the camera field-of-view where a digital image of the exposed surface was taken. The high 
contrast of the black/white embedded bone specimen, the uniform contrast of the entire field-of-view, and the sharp focus all ensure accurate imaging of the exposed trabecular microarchitecture (Figure 30). The image processing was fairly straightforward with uniform lighting and the high-contrast nature of the images. The threshold value was graphically chosen from the volume fraction-threshold value curve so that the volume fraction from serial milled images matches the Archimedes-based volume fraction (Beck et al., 1997). Percentage coefficient of variance was used for this comparison. Additionally, the slice-by-slice global thresholding technique was also validated by statistically comparing the structural characteristics ( $\mathrm{Tb} \mathrm{Th}, \mathrm{Tb} \mathrm{Sp} \& \mathrm{~Tb} \mathrm{Nm}$ ) from in vitro micro $\mathrm{CT}(\mathrm{CT})$ to serial milled images. T test-paired two samples for means and correlation was used for this comparison since the bone cubes measured in Archimedes are the same for serial milled and in vitro micro CT.

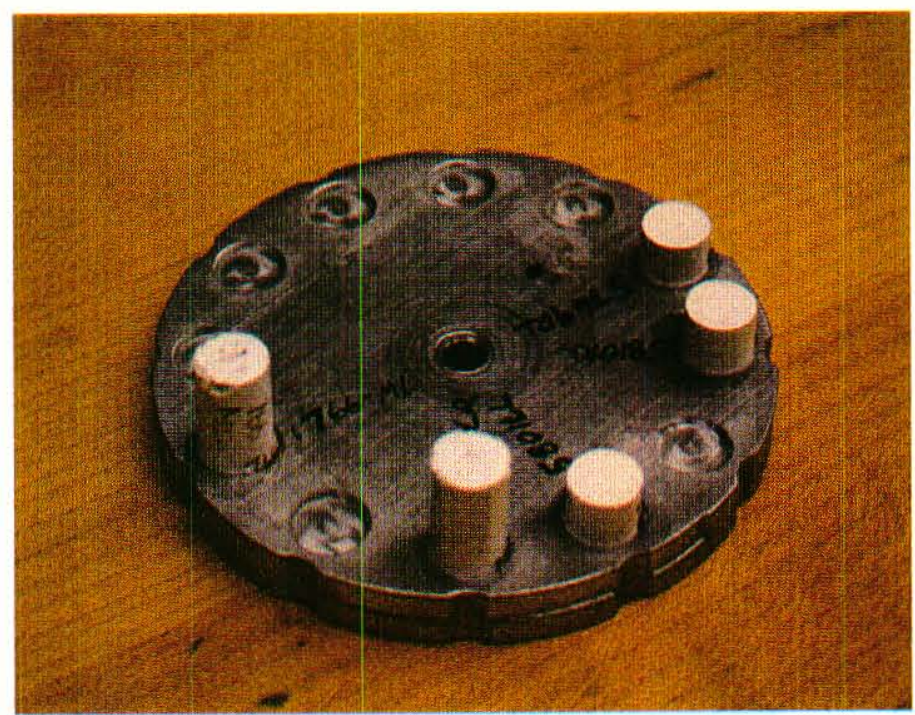

Figure 29. Circular 6061-T6 aluminum plate. 

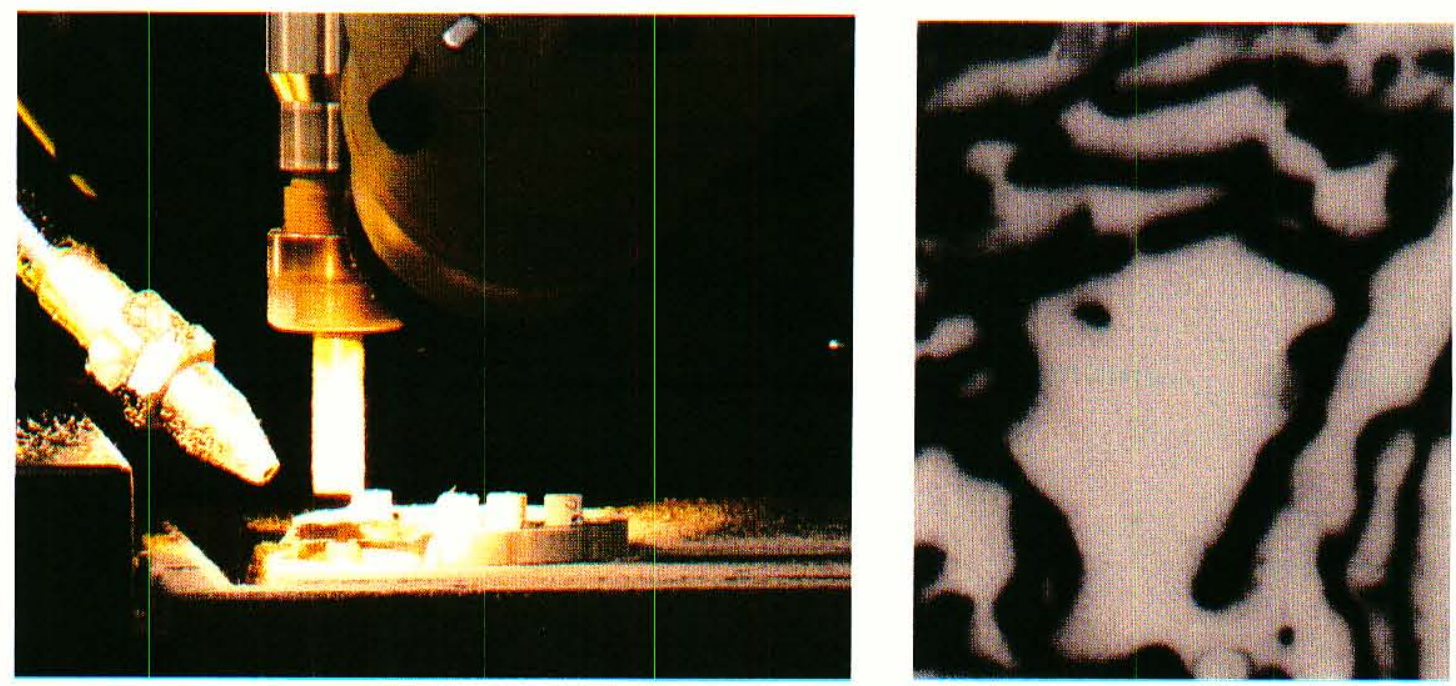

Figure 30. Serial Milling in process (left) and a serial milled image (right).

\section{Linear Elastic Finite Element Analysis}

In this part of the computational study, it is important to have the bone information contained within the image when directly converting from micro-CT images into microFEA model. A direct voxel conversion technique was applied using MatLab 7.0 (Program D - Appendix I) and Abaqus 6.5. All $14 \mu \mathrm{m}$ in vitro scans and $28 \mu \mathrm{m}$ in vivo scans were degraded to $56 \mu \mathrm{m}$ cubic voxels for micro-FE analysis due to memory limitations of the model during execution in Abaqus 6.5.

All micro-CT images were segmented before performing the process of direct voxel conversion into an FEA model. Each voxel was represented in rows and columns in each bone image. Therefore, the first step was to build a 3-D bone cube model, depending on the actual size of the bone cube (Figure 31). The boundary conditions applied to the bone model include (1) the base of the model was constrained in the z-direction (anteriorposterior), (2) a displacement of $0.4 \%$ strain was applied on the other side of the $\mathrm{z}$ direction for linear compressive testing, and (3) two edges of the base were constrained, one edge was constrained only in the $\mathrm{x}$-direction and the other edge was constrained only 
in the y-direction, such that rigid body motion was controlled. Each element was represented as one unit length in each direction of $\mathrm{x}, \mathrm{y}$ and $\mathrm{z}$. If the length (z-direction) of the model consisted of 80 , then a displacement of $0.4 \%$ strain of 80 would be equivalent to 0.32 units which was then used as the displacement applied to the model in the z-direction. This block of bone cube model was saved as an input file in Abaqus 6.5. The input file was viewed as text using TextPad 4.6 (Copyright (C) 1992-2003 Helios Software Solutions) where all elements were extracted from this input file and then saved with a new file name in txt format.

A custom written program in MatLab 7.0 (Program D - Appendix I) called upon the elements in this file to match the bone pixels from the images. If a bone pixel was not present at a specific location (column, row), then the element was eliminated; otherwise, the element that represented the bone pixel was saved in a new txt file. It was also important to take out the loose elements which were not connected to the main body of the bone block to avoid disappearance of elements which could lead to incomplete model execution. The connected voxels were grouped together in clusters based on six faces of a cubic voxel. If there was more than one cluster, the MatLab built-in function "bwlabeln" will eliminate the cluster, which has the least number of voxels that was not connected to the main body (cluster which has the maximum number of voxels). It is important to remove any elements which are not connected to the main body of the model because they were not properly constrained and could therefore exhibit physically unreasonable displacements leading to computational errors. When this was accomplished, the new input file containing only elements representing bone tissue was executed in Abaqus (Figure 32). 


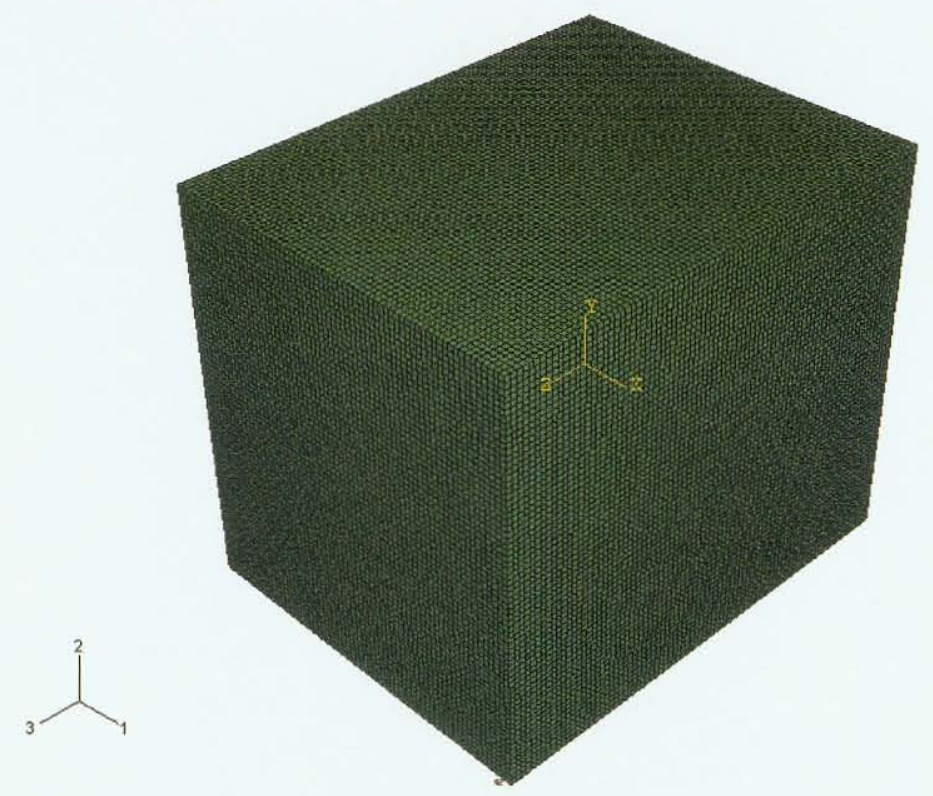

Figure 31. A bone cube model with bone and non-bone elements.

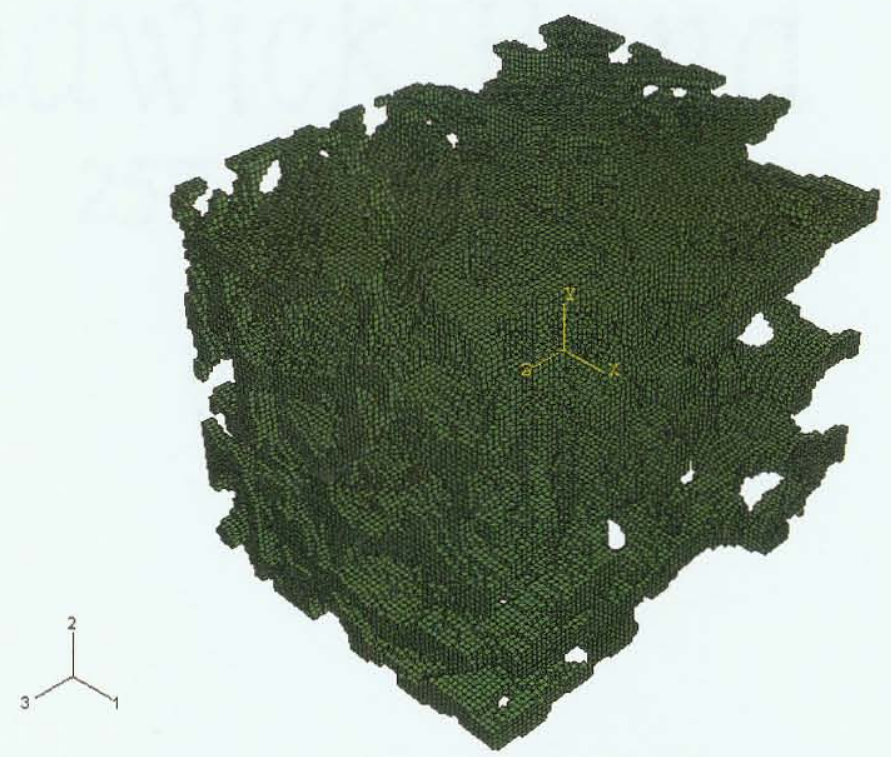

Figure 32. A bone cube model with only bone elements.

All bone cubes were scanned at $14 \mu \mathrm{m}$ nominal resolutions prior to mechanical testing such that these micro CT-based images could be directly converted to micro FEA models. The micro FEA models were executed using an arbitrary tissue modulus of 12 
$\mathrm{GPa}$ and the reaction forces in the z-direction was recorded in GPa. The cross-sectional area of the posterior surface was simply the number of voxels in the $\mathrm{x}$-direction multiplied by the number of voxels in the y-direction. The reaction forces of the posterior surface or essentially the base were reported in the results file which could be read using Notepad 5.1 (Copyright (c) 1981-2001 Microsoft Corporation) or TextPad 4.6. The stress value was then determined from the ratio of reaction forces $(F)$ to the crosssectional area (A) of the FEA model (stress = F / A). The arbitrary apparent stiffness value (AppS-arb) was obtained from the ratio of stress to strain (AppS = stress / strain). The apparent stiffness obtained from mechanical testing was used as a reference to derive apparent stiffness results in micro FEA models. The models were executed several times until correct tissue modulus was obtained. Since this is a linear model, the FEA apparent stiffness (AppS-FEA) could also be calculated by taking the ratio of apparent stiffness from compressive testing (AppS-MTS) to the arbitrary tissue modulus of $12 \mathrm{GPa}$ and multiplied by the AppS-arb (AppS-FEA = App-MTS / 12E9 * AppS-arb). The apparent stiffness determined from FEA models using the average correct tissue modulus were statistically compared to experimental results. T-test: paired two samples for means and correlation were used in this comparison.

\section{Mechanical Analysis (Compression Test)}

As all the rabbit bone specimens were cubes, they were tested between two parallel steel platens where strains were measured by a $25 \mathrm{~mm}$ gauge-length extensometer (model 632.31e.24, MTS, U.S.A.) attached to the platens. Two platens made of 304 stainless steel were machined (Figure 33) in the machine shop at the School of Medicine, 
University of Louisville. The surfaces of the platens were polished giving a smooth and flat surface for compression testing to reduce the existence of frictional end artifacts.
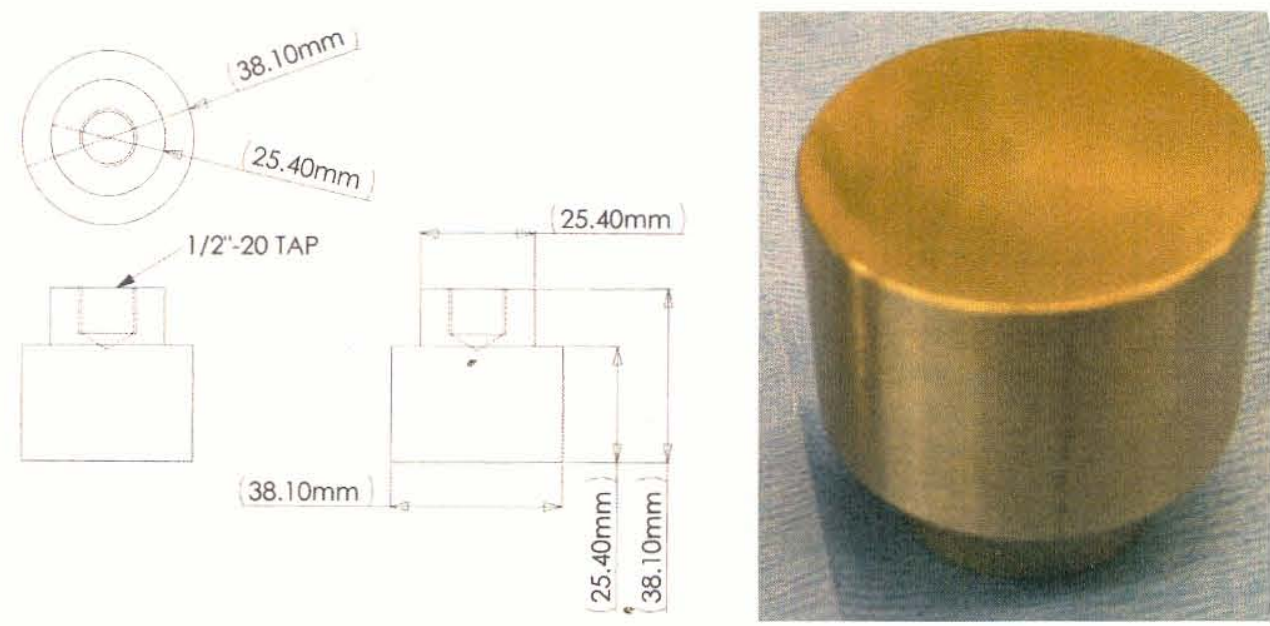

Figure 33. A 304 stainless steel platen

One platen was secured to the upper load cell and the other platen was secured to the base of the MTS machine. The surfaces of the platens were brushed with mineral oil to create a low friction boundary condition which is similar to the boundary condition applied in the FEA model. Additionally, lubrication reduced friction between specimen and platens that can contribute to measurement error. When a bone was compressed, it would expand in the transverse directions due to the Poisson's effect. Friction at the platen-specimen interface would interfere with this expansion and change the apparent stiffness of the specimen. A bone cube was placed on the lower platen and the upper platen was loaded to slightly touch the surface of the bone cube. The bone cube orientation was such that the load was applied in the anterior-posterior direction of the bone specimen. Then, a $25 \mathrm{~mm}$ gauge-length extensometer was attached to the platens with rubber bands (Figure 34). The force and the strain were zeroed prior to testing. 


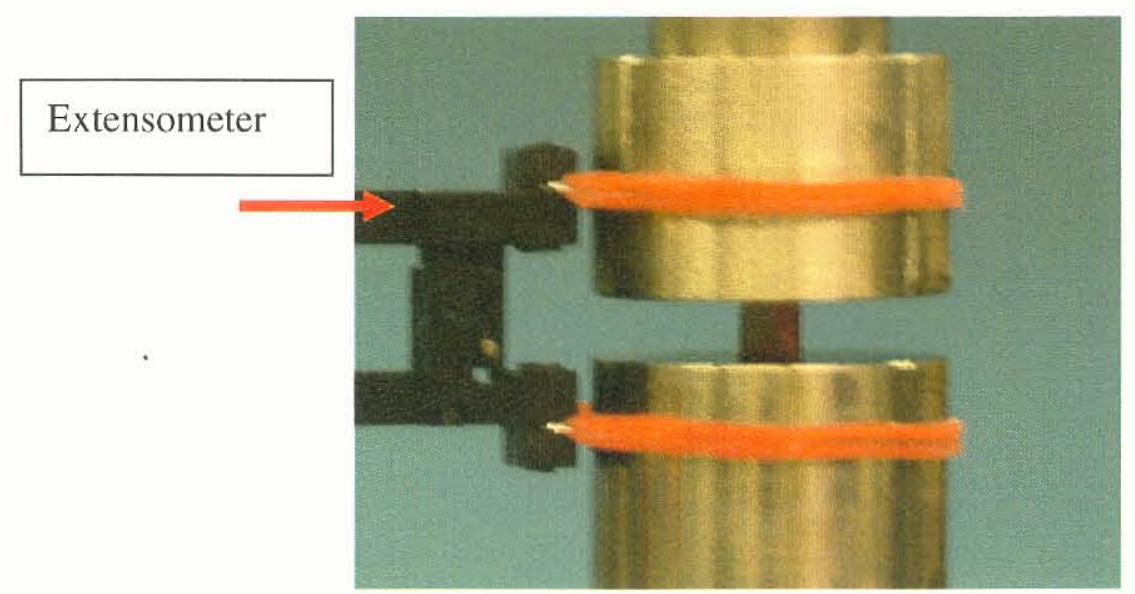

Figure 34. A close up view of a bone cube in between platens. A $25 \mathrm{~mm}$ gauge-length extensometer was attached to the platens with rubber bands

The following protocol is the MTS program for the compression testing of the rabbit bones. To eliminate effects of machine compliance, the test set up used strain as a control channel where strains were recorded by the 1 inch $(25.4 \mathrm{~mm})$ extensometer. This was due to the sensitivity of the LVDT on the MTS machine that has an error of $\pm 2 \%$ of the smallest range while the extensometer has an error of $\pm 0.3 \%$ of the maximum range and the error is much less between $0-60 \%$ of the full scale range. A compressive preload of 5-10N was first applied and the strains were zeroed. Then, a uniaxial compression preconditioning of 0 to $0.4 \%$ strain for 20 cycles was used to minimize the effects of end artifacts (Odgaard and Linde, 1991) and followed by a destructive ramp of up to $5 \%$ strain. A specific program (ramp test) was written that consisted of four specific processing steps which include:
a. Data Acquisition Parameters [data]
b. Cyclic Command Parameters [compression]
c. Monotonic Command Parameters [destructive]
d. Step Done [end of test] 
In the $1^{\text {st }}$ process [data], a time increment of $0.1 \mathrm{sec}$ was assigned, as this was a slow ramp test so as to capture the data at that rate of testing. The slow ramp test was to insure that the bone was not damaged especially during the preconditioning. In the $2^{\text {nd }}$ process [compression], a rate of $0.001 \mathrm{~mm} / \mathrm{sec}$ was assigned with an end level ramp rate of $-0.4 \%$ strain maintaining the bone intact in its elastic range. There was no specific data on the percentage of strain for rabbit bone in its elastic range. Therefore, the $0.4 \%$ strain was referenced from studies performed on bovine bone which maintained in its elastic region (Keaveny, et al. 1997). This number of $0.4 \%$ strain was chosen after performing trial tests on six rabbit bones samples prior to the rabbit bone samples for this study. As it reached the end of the cycle [destructive], a destructive ramp of up to 5\% strain at a rate of $500 \mathrm{microstrain} / \mathrm{sec}$, simulating a quasistatic condition, was applied to the bone sample. Finally, the program ended [end of test] when the level of $4.75 \%$ strain was reached. All strain, force and axial data were collected during the testing and analyzed using the Microsoft Excel spreadsheet program.

Stress $(\mathrm{Pa})$ was calculated as the ratio of reported force $(\mathrm{N})$ value to the bone cube cross-sectional area $\left(\mathrm{m}^{2}\right)$. The cross-sectional area was the surface area of the bone against the platens. Strain was measured as the ratio of the reported change in length to the total length of the bone cube at which it was compressed. The stress and strain curves were plotted for each sample (Data - Appendix I). The slope of the stress-strain curve within the elastic region was calculated giving the apparent stiffness of the trabecular bone (Figure 35). The apparent stiffness from the mechanical compressive test was statistically compared to the FEA apparent stiffness. T-test: paired two samples for means and correlation were used in this comparison. 
Stress-Strain Curve

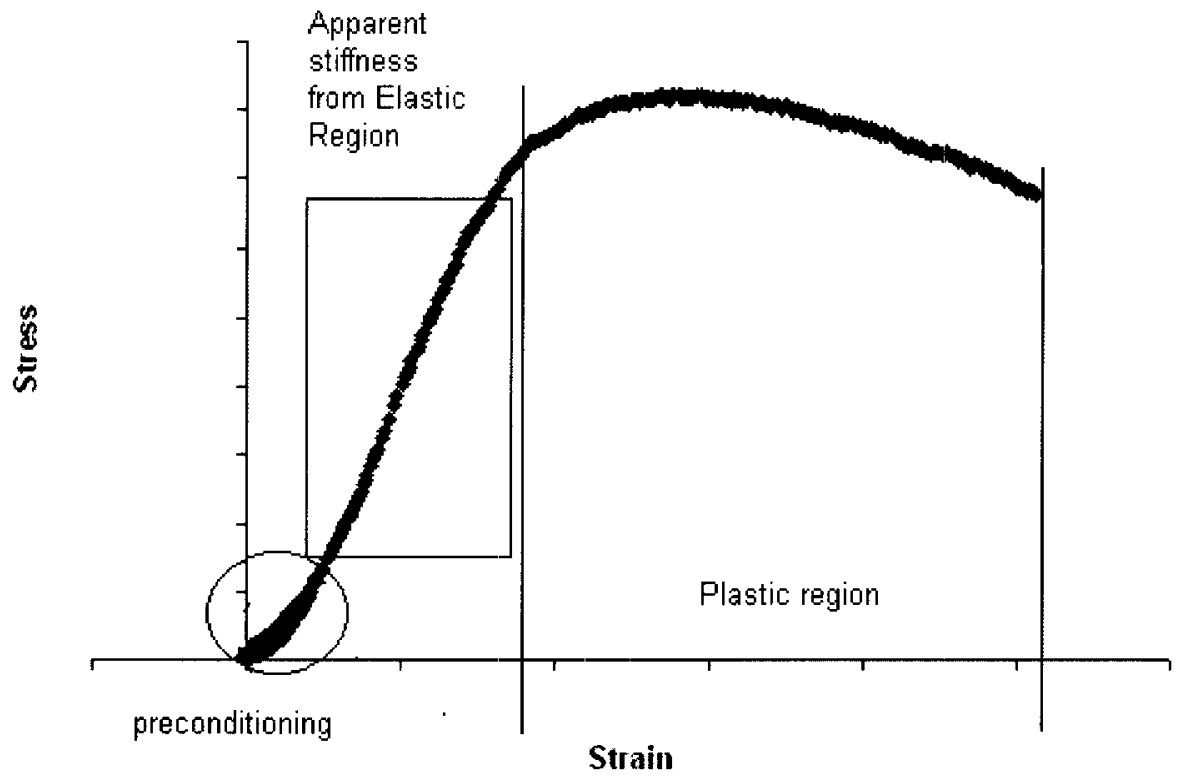

Figure 35. A stress-strain curve, illustrating preconditioning, apparent stiffness in the elastic region and load to failure in the plastic region. 


\section{RESULTS}

\section{Micro CT: In Vivo (T1) to In Vivo (T7)}

The volume fraction (VF), trabecular thickness ( $\mathrm{Tb} \mathrm{Th}$ ), trabecular separation ( $\mathrm{Tb} \mathrm{Sp}$ ) and trabecular number ( $\mathrm{Tb} \mathrm{Nm}$ ) for the in vivo scans at day-one (T1) and day-seven (T7) are given in Table 1 for a total of twelve bone cube specimens. These volumes of interest (VOIs) taken from three rabbits represent the left lateral (LL), right lateral (RL), left medial (LM) and right medial (RM) femoral condyles (Figure 36). At both T1 and T7 the VF, $\mathrm{Tb} \mathrm{Th}$ and $\mathrm{Tb} \mathrm{Nm}$ of the lateral condyles were lower than those of the medial condyles (Table 1). Similarly, the Tb Sp of the lateral condyles was higher than that of the medial condyles for $\mathrm{T} 1$ and $\mathrm{T} 7$. The VF, $\mathrm{Tb} \mathrm{Th}, \mathrm{Tb} \mathrm{Sp}$ and $\mathrm{Tb} \mathrm{Nm}$ for lateral and medial condyles were not significantly different when compared between $\mathrm{T} 1$ and $\mathrm{T} 7 \mathrm{p}=$ $0.32, p=0.49, p=1, p=0.83$ for lateral condyles and $p=0.43, p=0.39, p=0.51, p=$ 0.47 for medial condyles, respectively). The r-values or correlation coefficients for VF, $\mathrm{Tb} \mathrm{Th}$ and $\mathrm{Tb} \mathrm{Sp}$ between $\mathrm{T} 1$ and $\mathrm{T} 7$ were $0.99,0.76$ and 0.99 respectively (Figure 37). The average difference for $\mathrm{VF}, \mathrm{Tb} \mathrm{Th}$, and $\mathrm{Tb} \mathrm{Sp}$ of lateral and medial condyles between $\mathrm{T} 1$ and $\mathrm{T} 7$ were $1.09 \%, 1.70 \%, 0.91 \%,-0.4 \%,-1.6 \%$, and $0.9 \%$, respectively (Figure 38). The r-value and the average difference for $\mathrm{Tb} \mathrm{Nm}$ of lateral and medial condyles between $\mathrm{T} 1$ and $\mathrm{T} 7$ were $0.93,1.10 \%$ and $1.4 \%$ respectively. 


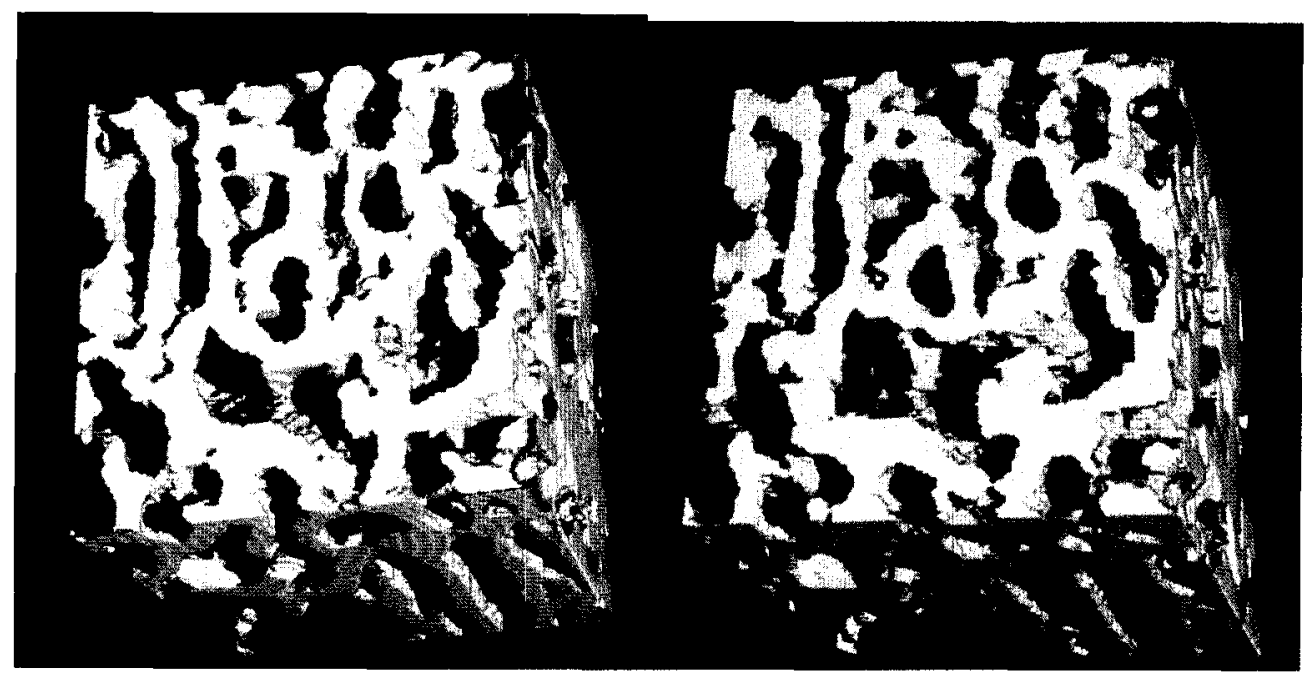

Figure 36. Volume of interests for T1 (left) and T7 (right) presented in three dimensional image stacks after slice-by-slice global threshold.

Table 1 VF, TbTh, TbSp and TbNm for In Vivo (T1) and In Vivo (T7) micro CT.

\section{T1}

\begin{tabular}{|c|c|c|c|c|c|c|c|c|c|}
\hline $\begin{array}{l}\text { rabbit bone } \\
\text { lateral } \\
\text { condyles } \\
\end{array}$ & VF & $\begin{array}{l}\text { Tb Th } \\
\mathrm{mm}\end{array}$ & $\begin{array}{l}\text { Tb } \mathrm{Nm} \\
\mathrm{mm}^{-1}\end{array}$ & $\begin{array}{l}\text { Tb Sp } \\
\mathrm{mm}\end{array}$ & $\begin{array}{l}\text { rabbit bone } \\
\text { medial } \\
\text { condyles }\end{array}$ & VF & $\begin{array}{l}\text { Tb Th } \\
\mathrm{mm}\end{array}$ & $\begin{array}{l}\text { Tb Nm } \\
\mathrm{mm}^{-1}\end{array}$ & $\begin{array}{l}\text { Tb Sp } \\
\mathrm{mm}\end{array}$ \\
\hline 721LL_T1 & 0.419 & 0.170 & 2.467 & 0.235 & 721LM_T1 & 0.501 & 0.183 & 2.733 & 0.183 \\
\hline 721RL_T1 & 0.401 & 0.183 & 2.196 & 0.273 & 721RM_T1 & 0.507 & 0.180 & 2.817 & 0.175 \\
\hline $720 L_{-}{ }_{1} 1$ & 0.414 & 0.150 & 2.760 & 0.212 & 720LM_T1 & 0.525 & 0.193 & 2.725 & 0.174 \\
\hline 720RL_T1 & 0.401 & 0.170 & 2.355 & 0.255 & 720RM_T1 & 0.526 & 0.181 & 2.915 & 0.163 \\
\hline 719LL_T1 & 0.389 & 0.174 & 2.245 & 0.272 & 719LM_T1 & 0.508 & 0.178 & 2.849 & 0.173 \\
\hline 719RL T1 & 0.379 & 0.176 & 2.154 & 0.288 & 719RM_T1 & 0.511 & 0.182 & 2.807 & 0.174 \\
\hline AVERAGE & 0.401 & 0.171 & 2.363 & 0.256 & AVERAGE & 0.513 & 0.183 & 2.808 & 0.174 \\
\hline STDEV & 0.015 & 0.011 & 0.225 & 0.028 & STDEV & 0.010 & 0.005 & 0.072 & 0.006 \\
\hline
\end{tabular}

T7

\begin{tabular}{|c|c|c|c|c|c|c|c|c|c|}
\hline $\begin{array}{l}\text { rabbit bone } \\
\text { lateral } \\
\text { condyles }\end{array}$ & VF & $\begin{array}{l}\text { Tb Th } \\
\mathrm{mm}\end{array}$ & $\begin{array}{l}\mathrm{Tb} \mathrm{Nm} \\
\mathrm{mm}^{-1}\end{array}$ & $\begin{array}{l}\text { Tb Sp } \\
\mathrm{mm}\end{array}$ & $\begin{array}{l}\text { rabbit bone } \\
\text { medial } \\
\text { condyles }\end{array}$ & VF & $\begin{array}{l}\text { Tb Th } \\
\mathrm{mm}\end{array}$ & $\begin{array}{l}\text { Tb Nm } \\
\mathrm{mm}^{-1}\end{array}$ & $\begin{array}{l}\text { Tb Sp } \\
\mathrm{Mm}\end{array}$ \\
\hline 721LL_T7 & 0.415 & 0.172 & 2.410 & 0.243 & 721LM_T7 & 0.499 & 0.183 & 2.722 & 0.184 \\
\hline 721RL_T7 & 0.401 & 0.182 & 2.204 & 0.272 & 721RM_T7 & 0.518 & 0.195 & 2.650 & 0.182 \\
\hline 720LL_T7 & 0.411 & 0.153 & 2.691 & 0.219 & 720LM_T7 & 0.521 & 0.196 & 2.667 & 0.180 \\
\hline 720RL_T7 & 0.411 & 0.177 & 2.323 & 0.253 & 720RM_T7 & 0.530 & 0.187 & 2.839 & 0.165 \\
\hline 719LL_T7 & 0.374 & 0.159 & 2.350 & 0.266 & 719LM_T7 & 0.513 & 0.185 & 2.774 & 0.176 \\
\hline 719RL T7 & 0.367 & 0.164 & 2.240 & 0.282 & 719RM_T7 & 0.509 & 0.171 & 2.977 & 0.165 \\
\hline AVERAGE & 0.397 & 0.168 & 2.370 & 0.256 & AVERAGE & 0.515 & 0.186 & 2.772 & 0.175 \\
\hline STDEV & 0.021 & 0.011 & 0.174 & 0.023 & STDEV & 0.011 & 0.009 & 0.122 & 0.008 \\
\hline
\end{tabular}




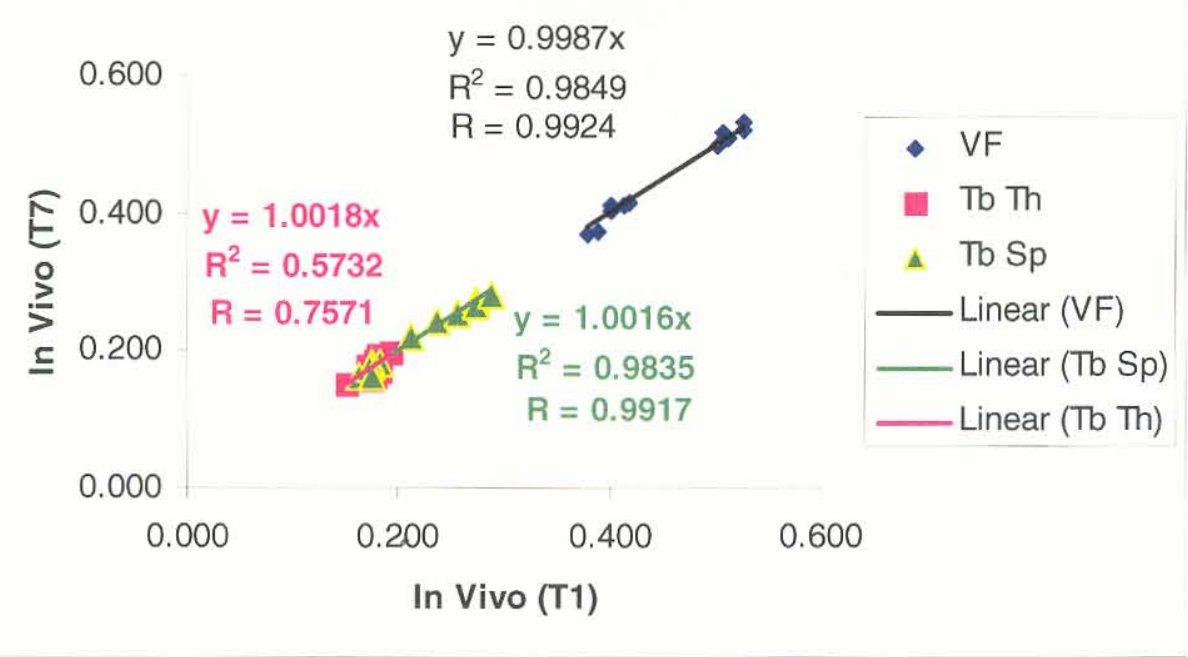

Figure 37. Volume fraction (VF), trabecular thickness ( $\mathrm{Tb} \mathrm{Th}$ ) and trabecular separation ( $\mathrm{Tb} \mathrm{Sp}$ ) of twelve bone cube specimens (medial and lateral condyles) measured in vivo (T1) were plotted against in vivo (T7). The $\mathrm{x}$ - and $\mathrm{y}$ - axes are unitless for VF and $\mathrm{mm}$ for $\mathrm{Tb} \mathrm{Th}$ and $\mathrm{Tb} \mathrm{Sp}$.

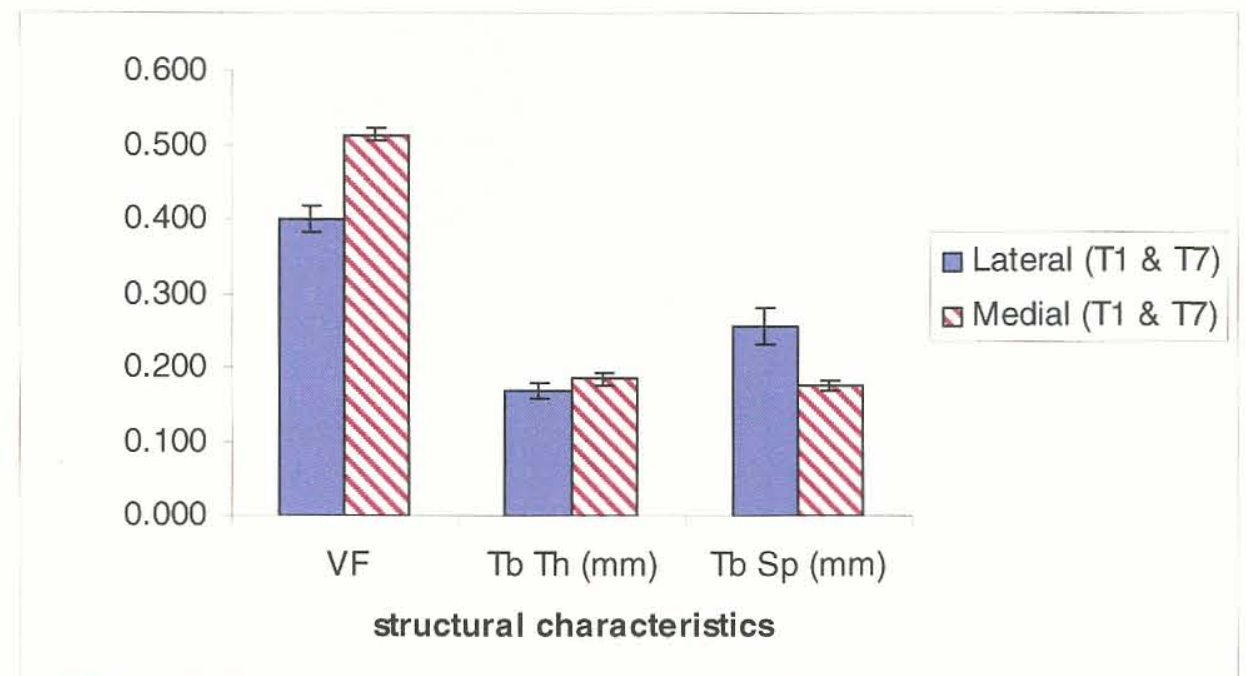

Figure 38. Lateral-medial $(\mathrm{T} 1 \& \mathrm{~T} 7)$ average values shown with standard deviation $(\mathrm{n}=$ 12) for volume fraction (VF), trabecular thickness (Tb $\mathrm{Th}$ ) and trabecular separation (Tb $\mathrm{Sp}$ ) of rabbit distal femur. The $\mathrm{y}$-axis is unitless for $\mathrm{VF}$ and $\mathrm{mm}$ for $\mathrm{Tb} \mathrm{Th}$ and $\mathrm{Tb} \mathrm{Sp}$. 


\section{Micro CT: In Vivo (T7') to In Vitro (Vit)}

The volume fraction (VF), trabecular thickness (Tb Th), trabecular separation ( $\mathrm{Tb} \mathrm{Sp}$ ) and trabecular number (Tb Nm) of In Vivo (T7') and In Vitro (Vit) scans (Figure 39) are given in Table 2 for a total of twelve bone cube specimens. These specimens were previously scanned at day-one and day-seven. The in vivo (T7') scan is actually the same as the in vivo (T7) scan but the volume of interest analyzed from the T7' scan is different from the $\mathrm{T} 7 \mathrm{scan}$. The average VF and $\mathrm{Tb}$ Th for the lateral and medial condyles of $\mathrm{T} 7$ ' were significantly higher than Vit values (Table 2, $\mathrm{p}<0.05$, Figure 40 ). Meanwhile, the average $\mathrm{Tb} \mathrm{Nm}$ for lateral condyles of $\mathrm{T} 7$ ' was higher than Vit but the average $\mathrm{Tb} \mathrm{Nm}$ for medial condyles of $\mathrm{T}^{\prime}$ ' was lower than Vit (Table 2). The $\mathrm{Tb} \mathrm{Nm}$ for lateral and medial condyles between T7' and Vit were not significantly different $(p=0.94, p=0.14)$ (Figure 40). The average $\mathrm{Tb} \mathrm{Sp}$ for both lateral and medial condyles of $\mathrm{T} 7$ ' was lower than Vit (Table 2). The $\mathrm{Tb} \mathrm{Sp}$ for the medial condyles between $\mathrm{T} 7$ ' and Vit were not significantly different $(\mathrm{p}=0.06)$ but the $\mathrm{Tb} \mathrm{Sp}$ for the lateral condyles between $\mathrm{T} 7$ ' and Vit was significantly different $(\mathrm{p}<0.05)$ (Figure 40$)$. The average differences of VF and $\mathrm{Tb} \mathrm{Th}$ of lateral condyles between $\mathrm{T} 7$, and Vit were $15.4 \%$ and $15.1 \%$, respectively and these values were roughly twice the average differences for the $\mathrm{VF}$ and $\mathrm{Tb}$ Th of the medial condyles between $\mathrm{T} 7$ and Vit, which were $6.6 \%$ and $9.8 \%$, respectively (Figure 40 ). It not only demonstrated an overestimation of the bone volume for the in vivo scans compared to the higher resolution in vitro scans, but the prediction also showed a greater $\%$ difference in the less dense bone of the lateral condyle compared to denser bone of the medial condyle. The r-values for $\mathrm{VF}, \mathrm{Tb} \mathrm{Sp}$ and $\mathrm{Tb} \mathrm{Th}$ for medial and lateral condyles between $T 7$ ' and Vit were $0.97,0.96$ and 0.66 respectively (Figure 41 ). 


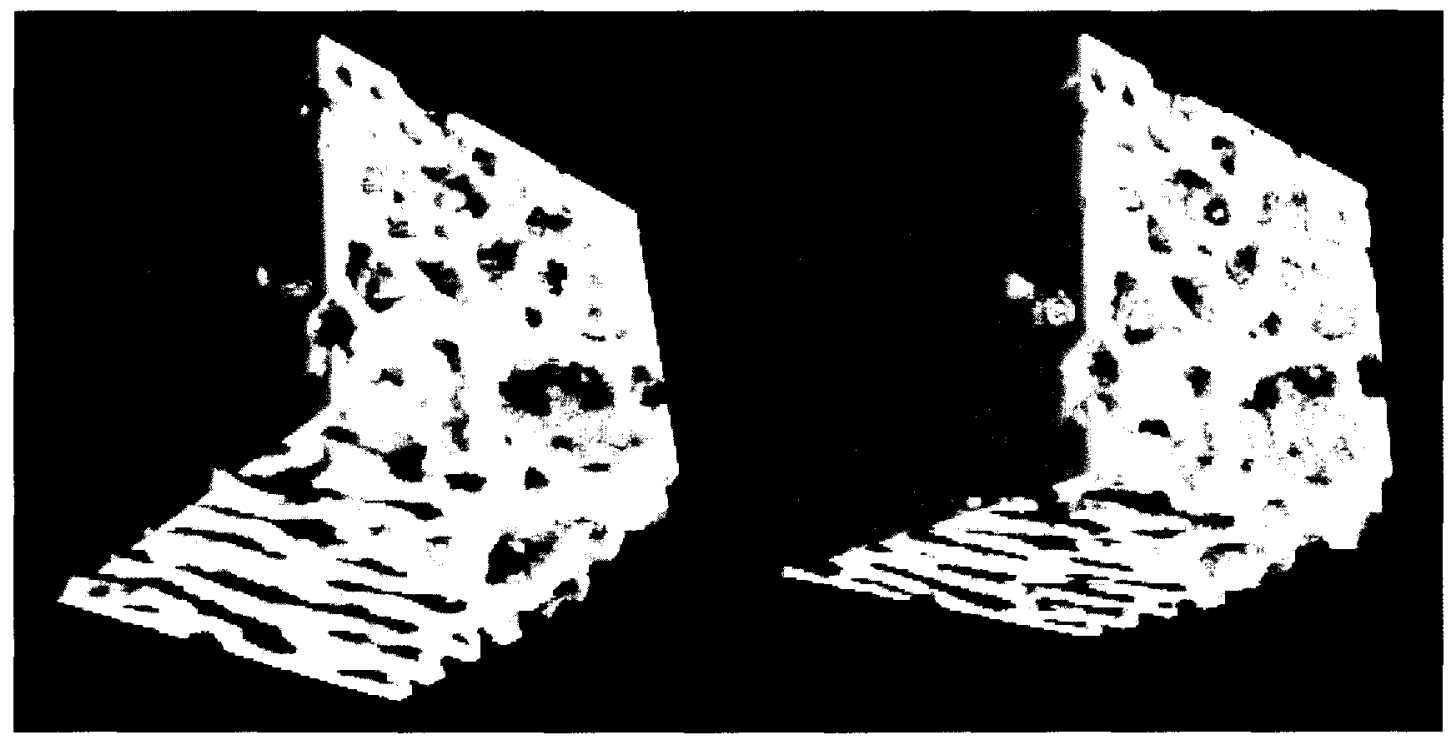

Figure 39. Volume of interests for T7' (left) and Vit (right) presented in three dimensional image stacks after slice-by-slice global threshold.

Table 2. $\mathrm{VF}, \mathrm{Tb} \mathrm{Th}, \mathrm{Tb} \mathrm{Nm}$ and $\mathrm{Tb} \mathrm{Sp}$ for in vivo (T7') and in vitro (Vit)

\begin{tabular}{|c|c|c|c|c|c|c|c|c|c|}
\hline $\begin{array}{l}\text { rabbit bone } \\
\text { lateral condyles }\end{array}$ & VF & $\begin{array}{l}\mathrm{Tb} \mathrm{Th} \\
\mathrm{mm}\end{array}$ & $\begin{array}{l}\mathrm{Tb} \mathrm{Nm} \\
\mathrm{mm}^{-1}\end{array}$ & $\begin{array}{l}\text { Tb Sp } \\
\mathrm{mm}\end{array}$ & $\begin{array}{l}\text { rabbit bone } \\
\text { medial condyles }\end{array}$ & $\mathrm{VF}$ & $\begin{array}{l}\text { Tb Th } \\
\mathrm{mm}\end{array}$ & $\begin{array}{l}\mathrm{Tb} \mathrm{Nm} \\
\mathrm{mm}^{-1}\end{array}$ & $\begin{array}{l}\text { Tb Sp } \\
\mathrm{mm}\end{array}$ \\
\hline $721 \mathrm{LL} \_T 7^{\prime}$ & 0.405 & 0.172 & 2.355 & 0.253 & 721LM_T7' & 0.498 & 0.177 & 2.812 & 0.178 \\
\hline $721 \mathrm{RL} \_T 7^{\prime}$ & 0.399 & 0.167 & 2.391 & 0.251 & 721RM_T7' & 0.532 & 0.178 & 2.986 & 0.157 \\
\hline 720LL_T7' & 0.375 & 0.155 & 2.419 & 0.259 & 720LM_T7' & 0.534 & 0.193 & 2.762 & 0.169 \\
\hline 720RL_T7' & 0.436 & 0.175 & 2.488 & 0.226 & 720RM_T7' & 0.530 & 0.187 & 2.835 & 0.166 \\
\hline 719LL_T7' & 0.382 & 0.159 & 2.408 & 0.256 & 719LM_T7' & 0.496 & 0.172 & 2.887 & 0.175 \\
\hline 719RL_T7' & 0.410 & 0.161 & 2.554 & 0.231 & 719RM_T7' & 0.507 & 0.167 & 3.033 & 0.163 \\
\hline AVERAGE & 0.401 & 0.165 & 2.436 & 0.246 & AVERAGE & 0.516 & 0.179 & 2.886 & 0.168 \\
\hline STDEV & 0.022 & 0.008 & 0.073 & 0.014 & STDEV & 0.018 & 0.010 & 0.105 & 0.008 \\
\hline \multicolumn{10}{|l|}{ Vit } \\
\hline $\begin{array}{l}\text { rabbit bone } \\
\text { lateral condyles }\end{array}$ & VF & $\begin{array}{l}\mathrm{Tb} T h \\
\mathrm{~mm}\end{array}$ & $\begin{array}{l}\mathrm{Tb} \mathrm{Nm} \\
\mathrm{mm}^{-1}\end{array}$ & $\begin{array}{l}\text { Tb Sp } \\
\mathrm{mm}\end{array}$ & $\begin{array}{l}\text { rabbit bone } \\
\text { medial condyles }\end{array}$ & $\mathrm{VF}$ & $\begin{array}{l}\text { Tb Th } \\
\mathrm{mm}\end{array}$ & $\begin{array}{l}\mathrm{Tb} \mathrm{Nm} \\
\mathrm{mm}^{-1}\end{array}$ & $\begin{array}{l}\text { Tb Sp } \\
\mathrm{mm}\end{array}$ \\
\hline 721LL_Vit & 0.350 & 0.137 & 2.562 & 0.254 & 721LM_Vit & 0.459 & 0.153 & 2.996 & 0.181 \\
\hline 721RL_Vit & 0.339 & 0.141 & 2.394 & 0.276 & 721RM_Vit & 0.491 & 0.155 & 3.172 & 0.161 \\
\hline 720LL_Vit & 0.322 & 0.135 & 2.390 & 0.284 & 720LM_Vit & 0.517 & 0.181 & 2.860 & 0.169 \\
\hline 720RL_Vit & 0.380 & 0.146 & 2.612 & 0.237 & 720RM_Vit & 0.494 & 0.164 & 3.006 & 0.168 \\
\hline 719LL_Vit & 0.336 & 0.158 & 2.135 & 0.311 & 719LM_Vit & 0.473 & 0.17 & 2.784 & 0.189 \\
\hline 719RL_Vit & 0.360 & 0.145 & 2.491 & 0.257 & 719RM_Vit & 0.473 & 0.157 & 3.015 & 0.175 \\
\hline AVERAGE & 0.348 & 0.144 & 2.431 & 0.270 & AVERAGE & 0.485 & 0.163 & 2.972 & 0.174 \\
\hline STDEV & 0.020 & 0.008 & 0.170 & 0.026 & STDEV & 0.021 & 0.011 & 0.135 & 0.010 \\
\hline
\end{tabular}




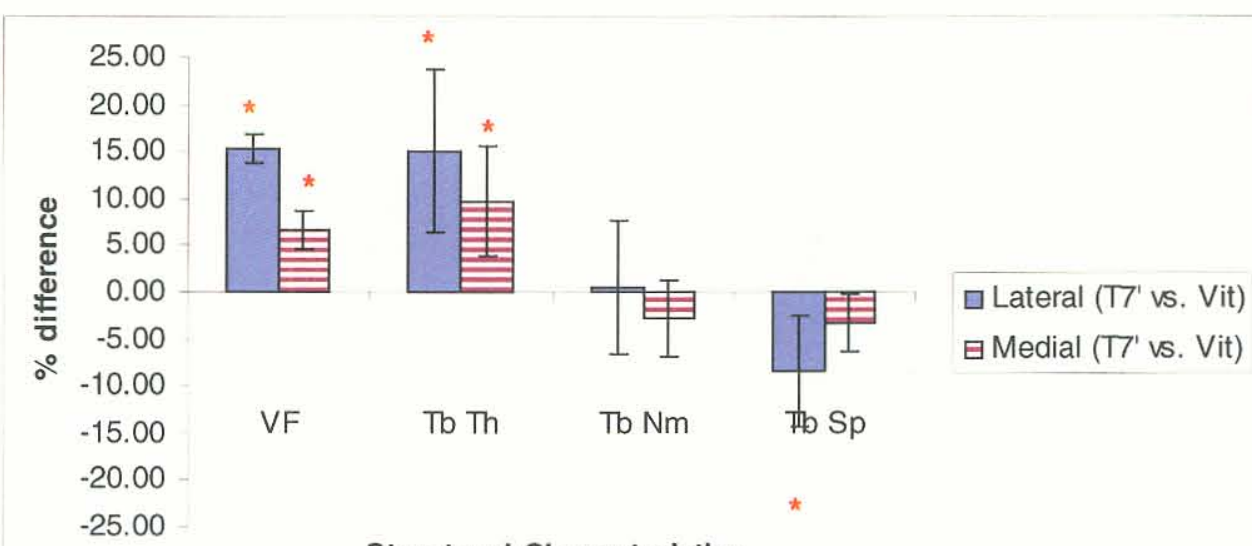

Structural Characteristics

Figure 40. Lateral-medial (T7 ${ }^{\prime}$ vs. Vit) average \% differences shown with standard deviation $(n=12)$ for volume fraction $(\mathrm{VF})$, trabecular thickness $(\mathrm{Tb} \mathrm{Th})$, trabecular

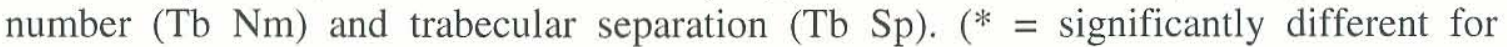
$\mathrm{p}<0.05$ t-Tests: Paired Two Sample for Means)

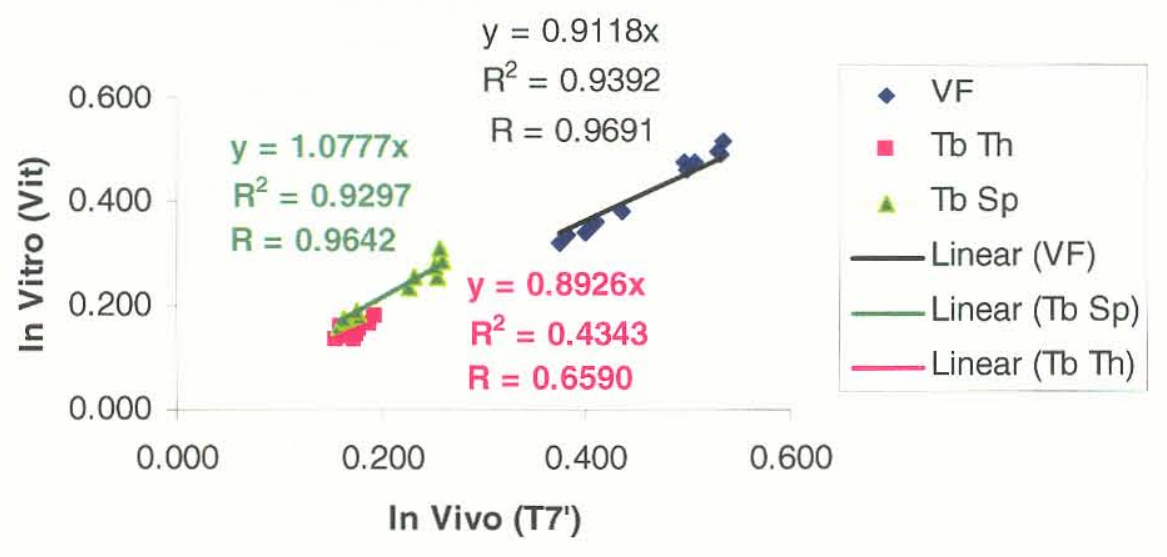

Figure 41. Volume fraction (VF), trabecular thickness (Tb Th) and trabecular separation ( $\mathrm{Tb} \mathrm{Sp}$ ) of twelve bone cube specimens (medial and lateral condyles) measured in vivo (T7') were plotted against in vitro (Vit). The $\mathrm{x}$ - and $\mathrm{y}$ - axes are unitless for VF and $\mathrm{mm}$ for $\mathrm{Tb} \mathrm{Th}$ and $\mathrm{Tb} \mathrm{Sp}$. 


\section{Micro CT: In Vivo with correlation factor (T7' CF)}

By applying the slice-by-slice global thresholding technique with an appropriate $\%$ correction factor $(\mathrm{CF})$ to each group of rabbit bones; the best case in vivo prediction of $\mathrm{VF}, \mathrm{Tb} \mathrm{Th}, \mathrm{Tb} \mathrm{Sp}$ and $\mathrm{Tb} \mathrm{Nm}$ was able to be determined. When the VF of $\mathrm{T} 7^{\prime} \mathrm{CF}$ matched the VF of Vit, the $\mathrm{Tb} \mathrm{Th}, \mathrm{Tb} \mathrm{Sp}$ and $\mathrm{Tb} \mathrm{Nm}$ were also corrected yielding a more accurate measurement between $\mathrm{T} 7{ }^{\prime} \mathrm{CF}$ and Vit (Table 3). The VF for the lateral and medial condyles between $\mathrm{T} 7^{\prime} \mathrm{CF}$ and Vit was expected to have a perfect correlation $(\mathrm{R}=$ 1) since the VF of T7'CF was forced to match the VF of Vit (Figure 42). The r-value for Tb Sp of the lateral and medial condyles between T7' CF and Vit was still above 0.96, while the r-value for $\mathrm{Tb} \mathrm{Th}$ of the lateral and medial condyles between $\mathrm{T} 7{ }^{\prime} \mathrm{CF}$ and Vit improved resulting in a better correlation (Figure 42). In addition, the r-value for $\mathrm{Tb} \mathrm{Nm}$ of the lateral and medial condyles between T7 ${ }^{\prime} \mathrm{CF}$ and Vit improved to 0.83 . After the correction factor was applied, the average \% difference between the in vivo (T7 $\left.{ }^{\prime} \mathrm{CF}\right)$ and in vitro (Vit) calculations of VF, $\mathrm{Tb} \mathrm{Th}, \mathrm{Tb} \mathrm{Nm}$ and $\mathrm{Tb} \mathrm{Sp}$ were all significantly reduced for the lateral condyles $(p=0.36, p=0.85, p=0.72 \& p=0.84)$ as well as the medial condyles ( $p=0.17, p=0.34, p=0.33 \& p=0.40$, respectively) (Figure 43). 
Table 3. VF, TbTh, TbSp and TbNm for T7' with correction factor (T7' CF) compared to Vit

\begin{tabular}{|c|c|c|c|c|c|c|c|c|c|c|}
\hline \multirow{2}{*}{$\begin{array}{l}\text { T7'CF } \\
\text { rabbit bone } \\
\text { Lateral } \\
\text { condyles }\end{array}$} & \multicolumn{10}{|c|}{ Vit } \\
\hline & VF & $\begin{array}{l}\text { Tb Th } \\
\mathrm{mm}\end{array}$ & $\begin{array}{l}\mathrm{Tb} \\
\mathrm{Sp} \\
\mathrm{mm} \\
\end{array}$ & $\begin{array}{l}\mathrm{Tb} \\
\mathrm{Nm} \\
\mathrm{mm}^{-1}\end{array}$ & $\begin{array}{l}\% \text { CF } \\
\text { applied } \\
\text { to T7' }\end{array}$ & $\begin{array}{l}\text { rabbit bone } \\
\text { lateral } \\
\text { condyles }\end{array}$ & VF & $\begin{array}{l}\text { Tb Th } \\
\mathrm{mm}\end{array}$ & $\begin{array}{l}\mathrm{Tb} \\
\mathrm{Sp} \\
\mathrm{mm} \\
\end{array}$ & $\begin{array}{l}\mathrm{Tb} \\
\mathrm{Nm} \\
\mathrm{mm}^{-1}\end{array}$ \\
\hline 721LL_T7'CF & 0.350 & 0.150 & 0.279 & 2.328 & 1.180 & 721LL_Vit & 0.350 & 0.137 & 0.254 & 2.562 \\
\hline 721RL_T7'CF & 0.339 & 0.144 & 0.28 & 2.362 & 1.200 & 721RL_Vit & 0.339 & 0.141 & 0.276 & 2.394 \\
\hline 720LL_T7'CF & 0.323 & 0.137 & 0.289 & 2.347 & 0.960 & 720LL_Vit & 0.322 & 0.135 & 0.284 & 2.390 \\
\hline 720RL_T7'CF & 0.380 & 0.153 & 0.249 & 2.486 & 1.020 & 720RL_Vit & 0.380 & 0.146 & 0.237 & 2.612 \\
\hline 719LL_T7'CF & 0.336 & 0.141 & 0.279 & 2.380 & 0.980 & 719LL_Vit & 0.336 & 0.158 & 0.311 & 2.135 \\
\hline 719RL_T7'CF & 0.360 & 0.142 & 0.253 & 2.532 & 1.020 & 719RL_Vit & 0.360 & 0.145 & 0.257 & 2.491 \\
\hline AVERAGE & 0.348 & 0.145 & 0.272 & 2.406 & 1.060 & AVERAGE & 0.348 & 0.144 & 0.270 & 2.431 \\
\hline STDEV & 0.02 & 0.006 & 0.016 & 0.083 & 0.001 & STDEV & 0.020 & 0.008 & 0.026 & 0.170 \\
\hline T7'CF & & & & & V & & & & & \\
\hline $\begin{array}{l}\text { rabbit bone } \\
\text { Medial } \\
\text { condyles }\end{array}$ & VF & $\begin{array}{l}\text { Tb Th } \\
\mathrm{mm}\end{array}$ & $\begin{array}{l}\mathrm{Tb} \\
\mathrm{Sp} \\
\mathrm{mm}\end{array}$ & $\begin{array}{l}\mathrm{Tb} \\
\mathrm{Nm} \\
\mathrm{mm}^{-1}\end{array}$ & $\begin{array}{l}\% \text { CF } \\
\text { applied } \\
\text { to T7' }\end{array}$ & $\begin{array}{l}\text { rabbit bone } \\
\text { medial } \\
\text { condyles }\end{array}$ & VF & $\begin{array}{l}\text { Tb Th } \\
\mathrm{mm}\end{array}$ & $\begin{array}{l}\mathrm{Tb} \\
\mathrm{Sp} \\
\mathrm{mm}\end{array}$ & $\begin{array}{l}\mathrm{Tb} \\
\mathrm{Nm} \\
\mathrm{mm}^{-1}\end{array}$ \\
\hline 721LM_T7'CF & 0.460 & 0.162 & 0.190 & 2.838 & .0 .660 & 721LM_Vit & 0.459 & 0.153 & 0.181 & 2.996 \\
\hline 721RM_T7'CF & 0.491 & 0.161 & 0.167 & 3.046 & 0.660 & 721RM_Vit & 0.491 & 0.155 & 0.161 & 3.172 \\
\hline 720LM_T7'CF & 0.518 & 0.186 & 0.173 & 2.79 & 0.300 & 720LM_Vit & 0.517 & 0.181 & 0.169 & 2.860 \\
\hline 720RM_T7'CF & 0.494 & 0.17 & 0.175 & 2.898 & 0.580 & 720RM_Vit & 0.494 & 0.164 & 0.168 & 3.006 \\
\hline 719LM_T7'CF & 0.473 & 0.163 & 0.181 & 2.903 & 0.380 & 719LM_Vit & 0.473 & 0.17 & 0.189 & 2.784 \\
\hline 719RM_T7'CF & 0.473 & 0.154 & 0.172 & 3.066 & 0.560 & 719RM_Vit & 0.473 & 0.157 & 0.175 & 3.015 \\
\hline AVERAGE & 0.485 & 0.166 & 0.176 & 2.924 & 0.523 & AVERAGE & 0.485 & 0.163 & 0.174 & 2.972 \\
\hline STDEV & 0.021 & 0.011 & 0.008 & 0.111 & 0.001 & STDEV & 0.021 & 0.011 & 0.010 & 0.135 \\
\hline
\end{tabular}

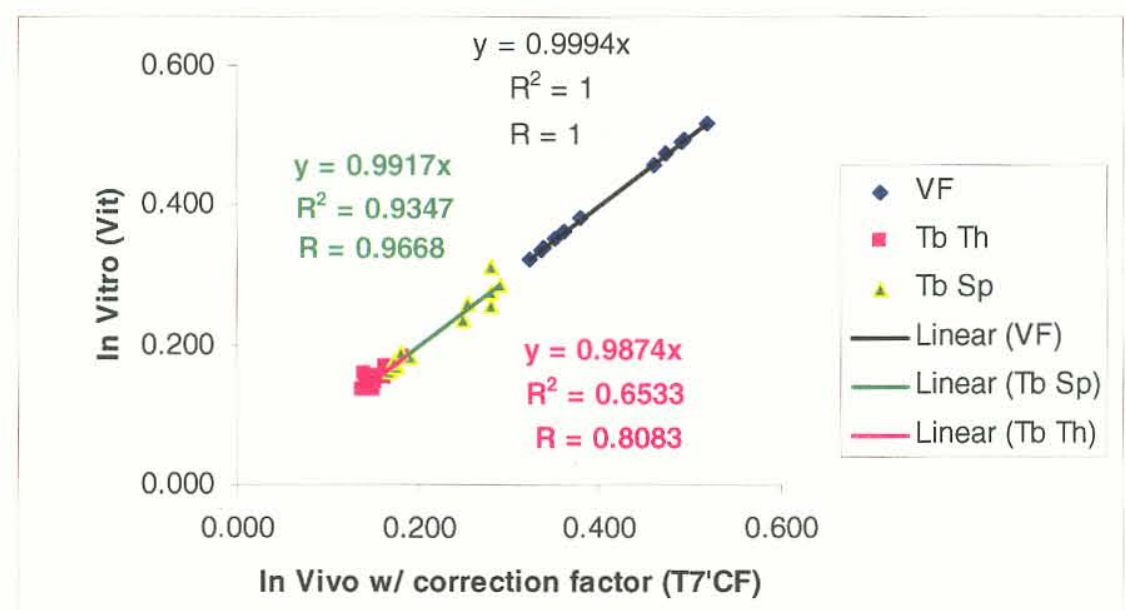

Figure 42. Volume fraction (VF), trabecular thickness (Tb $\mathrm{Th}$ ) and trabecular separation ( $\mathrm{Tb} \mathrm{Sp}$ ) of twelve bone cube specimens (medial and lateral condyles) measured in vivo with \% correction factor (T7' $\mathrm{CF}$ ) were plotted against in vitro (Vit). The $\mathrm{x}$ - and $\mathrm{y}$ - axes are unitless for $\mathrm{VF}$ and $\mathrm{mm}$ for $\mathrm{Tb} \mathrm{Th}$ and $\mathrm{Tb} \mathrm{Sp}$. 


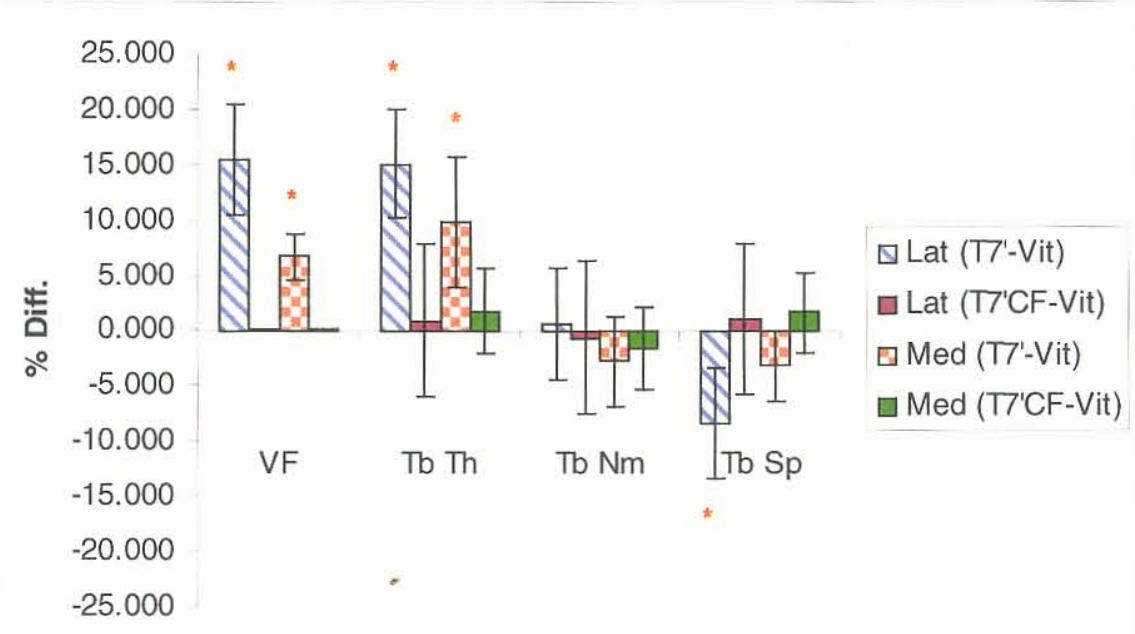

Figure 43. Lateral-medial (in vivo-T7' vs. in vitro-Vit) and lateral-medial (in vivo with correction factor-T7' $\mathrm{CF}$ vs. in vitro-Vit) average \% differences shown with standard deviation $(n=12)$ for volume fraction $\left(V^{*} F\right)$, trabecular thickness $(\mathrm{Tb} T h)$, trabecular number $(\mathrm{Tb} \mathrm{Nm})$ and trabecular separation $(\mathrm{Tb} \mathrm{Sp}) .(*$ = significantly different for $\mathrm{p}<0.05$ t-Tests: Paired Two Sample for Means) The VF, Tb Th, Tb Nm and Tb Sp for the lateral and medial condyles between T7' $\mathrm{CF}$ and. Vit were not significantly different $(\mathrm{p}=0.36, \mathrm{p}=0.85, \mathrm{p}=0.72 \& \mathrm{p}=0.84, \mathrm{p}=0.17, \mathrm{p}=0.34, \mathrm{p}=0.33 \& \mathrm{p}=0.40)$.

4. Micro CT: In Vivo (T1CF) to In Vivo (T7CF) with \% correction factor

The volume fraction (VF), trabecular thickness (Tb Th), trabecular spacing (Tb $\mathrm{Sp}$ ) and trabecular number $(\mathrm{Tb} \mathrm{Nm})$ for the in vivo scans $\mathrm{T} 1 \mathrm{CF}$ and $\mathrm{T} 7 \mathrm{CF}$, after \% correction factor was applied, are given in Table 4 for a total of twelve bone cube specimens (lateral and medial condyles). The r-values for $\mathrm{VF}, \mathrm{Tb} \mathrm{Sp}$ and $\mathrm{Tb} \mathrm{Th}$ of lateral and medial condyles between T1CF and T7CF were $0.99,0.99$ and 0.86 , respectively (Figure 44). Additionally, the correlation of $\mathrm{Tb} \mathrm{Nm}$ of lateral and medial condyles between $\mathrm{T} 1 \mathrm{CF}$ and T7CF increased $(R=0.95)$. Averages with standard deviation of VF, Tb Th and $\mathrm{Tb} \mathrm{Sp}$ for lateral and medial condyles between T1CF-T7CF and Vit were calculated and T-tests: Paired two samples for means were performed between T1CF-T7CF and Vit (Figure 45). 
The VF, Tb Nm and Tb Sp for lateral and medial condyles between T1CF-T7CF and Vit were not significantly different (lateral condyles; $p=0.429, p=0.110, p=0.148$, and medial condyles; $\mathrm{p}=0.602, \mathrm{p}=0.066, \mathrm{p}=0.107)$. The $\mathrm{Tb}$ Th for lateral condyles between $\mathrm{T} 1 \mathrm{CF}-$ T7CF and Vit were not significantly different $(p=0.193)$ but significantly different for medial condyles $(\mathrm{p}=0.027)$. However, the difference of $\mathrm{Tb}$ Th for medial condyles between T1CF-T7CF and Vit was only within $0.008 \mathrm{~mm} \pm 0.006$.

Table $4 \mathrm{VF}$, TbTh, TbSp and TbNm for in vivo with \% correction factor (T1CF \& T7CF)

\begin{tabular}{|c|c|c|c|c|c|c|c|c|c|c|}
\hline \multirow{2}{*}{$\begin{array}{l}\text { T1CF } \\
\text { rabbit bone } \\
\text { lateral } \\
\text { condyles } \\
\end{array}$} & \multicolumn{10}{|c|}{ T7CF } \\
\hline & VF & $\begin{array}{l}\text { Tb Th } \\
\mathrm{mm}\end{array}$ & $\begin{array}{l}\mathrm{Tb} \\
\mathrm{Nm} \\
\mathrm{mm}^{-1}\end{array}$ & $\begin{array}{l}\mathrm{Tb} \\
\mathrm{Sp} \\
\mathrm{mm}\end{array}$ & $\begin{array}{l}\text { \%CF } \\
\text { applied } \\
\text { to T1 \& } \\
T 7 \\
\end{array}$ & $\begin{array}{l}\text { rabbit bone } \\
\text { lateral condyles }\end{array}$ & VF & $\begin{array}{l}\text { Tb Th } \\
\mathrm{mm}\end{array}$ & $\begin{array}{l}\mathrm{Tb} \\
\mathrm{Nm} \\
\mathrm{mm}^{-1} \\
\end{array}$ & $\begin{array}{l}\mathrm{Tb} \\
\mathrm{Sp} \\
\mathrm{mm}\end{array}$ \\
\hline 721LL_T1CF & 0.369 & 0.151 & 2.450 & 0.258 & 1.180 & 721LL_T7CF & 0.366 & 0.154 & 2.383 & 0.266 \\
\hline 721RL_T1CF & 0.340 & 0.158 & 2.158 & 0.306 & 1.200 & 721RL_T7CF & 0.339 & 0.157 & 2.164 & 0.306 \\
\hline 720LL_T1CF & 0.321 & 0.133 & 2.421 & 0.281 & 0.960 & 720LL_T7CF & 0.324 & 0.136 & 2.382 & 0.284 \\
\hline 720RL_T1CF & 0.350 & 0.151 & 2.319 & 0.280 & 1.020 & 720RL_T7CF & 0.361 & 0.157 & 2.299 & 0.278 \\
\hline 719LL_T1CF & 0.339 & 0.153 & $2.21 \dagger$ & 0.299 & 0.980 & 719LL_T7CF & 0.332 & 0.143 & 2.321 & 0.288 \\
\hline 719RL_T1CF & 0.331 & 0.157 & 2.107 & 0.318 & 1.020 & 719RL_T7CF & 0.324 & 0.148 & 2.194 & 0.308 \\
\hline AVERAGE & 0.342 & 0.151 & 2.278 & 0.290 & 1.060 & AVERAGE & 0.341 & 0.149 & 2.291 & 0.288 \\
\hline STDEV & 0.017 & 0.009 & 0.141 & 0.022 & 0.001 & STDEV & 0.018 & 0.008 & 0.093 & 0.016 \\
\hline \multicolumn{6}{|l|}{ T1CF } & \multicolumn{5}{|l|}{ T7CF } \\
\hline $\begin{array}{l}\text { rabbit bone } \\
\text { medial } \\
\text { condyles }\end{array}$ & VF & $\begin{array}{l}\text { Tb Th } \\
\mathrm{mm}\end{array}$ & $\begin{array}{l}\mathrm{Tb} \\
\mathrm{Nm} \\
\mathrm{mm}^{-1} \\
\end{array}$ & $\begin{array}{l}\mathrm{Tb} \\
\mathrm{Sp} \\
\mathrm{mm}\end{array}$ & $\begin{array}{l}\text { \%CF } \\
\text { applied } \\
\text { to } \\
\mathrm{T} 1, T 7 \\
\end{array}$ & $\begin{array}{l}\text { rabbit bone } \\
\text { medial condyles }\end{array}$ & VF & $\begin{array}{l}\text { Tb Th } \\
\mathrm{mm}\end{array}$ & $\begin{array}{l}\mathrm{Tb} \\
\mathrm{Nm} \\
\mathrm{mm}^{-1}\end{array}$ & $\begin{array}{l}\mathrm{Tb} \\
\mathrm{Sp} \\
\mathrm{mm}\end{array}$ \\
\hline 721LM_T1CF & 0.462 & 0.167 & 2.769 & 0.194 & 0.660 & 721LM_T7CF & 0.462 & 0.168 & 2.751 & 0.196 \\
\hline 721RM_T1CF & 0.464 & 0.163 & 2.850 & 0.188 & 0.660 & 721RM_T7CF & 0.480 & 0.178 & 2.695 & 0.193 \\
\hline 720LM_T1CF & 0.507 & 0.184 & 2.752 & 0.179 & 0.300 & 720LM_T7CF & 0.505 & 0.188 & 2.691 & 0.184 \\
\hline 720RM_T1CF & 0.488 & 0.164 & 2.968 & 0.173 & 0.580 & 720RM_T7CF & 0.493 & 0.170 & 2.900 & 0.175 \\
\hline 719LM_T1CF & 0.480 & 0.168 & 2.862 & 0.182 & 0.380 & 719LM_T7CF & 0.493 & 0.176 & 2.798 & 0.181 \\
\hline 719RM_T1CF & 0.475 & 0.167 & 2.843 & 0.185 & 0.560 & 719RM_T7CF & 0.474 & 0.157 & 3.018 & 0.174 \\
\hline AVERAGE & 0.479 & 0.169 & 2.841 & 0.184 & 0.523 & AVERAGE & 0.485 & 0.173 & 2.809 & 0.184 \\
\hline STDEV & 0.017 & 0.008 & 0.077 & 0.007 & 0.001 & STDEV & 0.016 & 0.010 & 0.128 & 0.009 \\
\hline
\end{tabular}




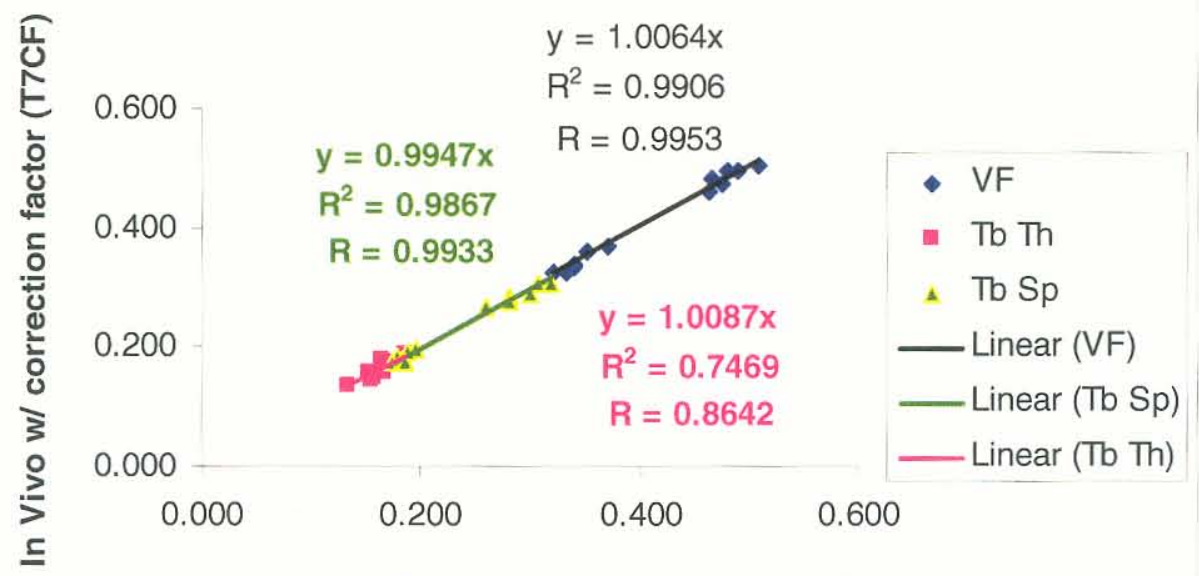

In Vivo wí correction factor (T1CF)

Figure 44. Volume fraction (VF), trabecular thickness ( $\mathrm{Tb} \mathrm{Th}$ ) and trabecular spacing ( $\mathrm{Tb}$ $\mathrm{Sp}$ ) of twelve bone cube specimens (medial and lateral condyles) measured in T1CF is plotted against T7CF. Correlation of VF between T1CF and T7CF was expected to be perfect while the $\mathrm{Tb} \mathrm{Sp}$ and $\mathrm{Tb} \mathrm{Th}$ between $\mathrm{T} 1 \mathrm{CF}$ and $\mathrm{T} 7 \mathrm{CF}$ were highly correlated. The $\mathrm{x}$ - and $\mathrm{y}$ - axes are unitless for $\mathrm{VF}$ and $\mathrm{mm}$ for $\mathrm{Tb} \mathrm{Th}$ and $\mathrm{Tb} \mathrm{Sp}$.

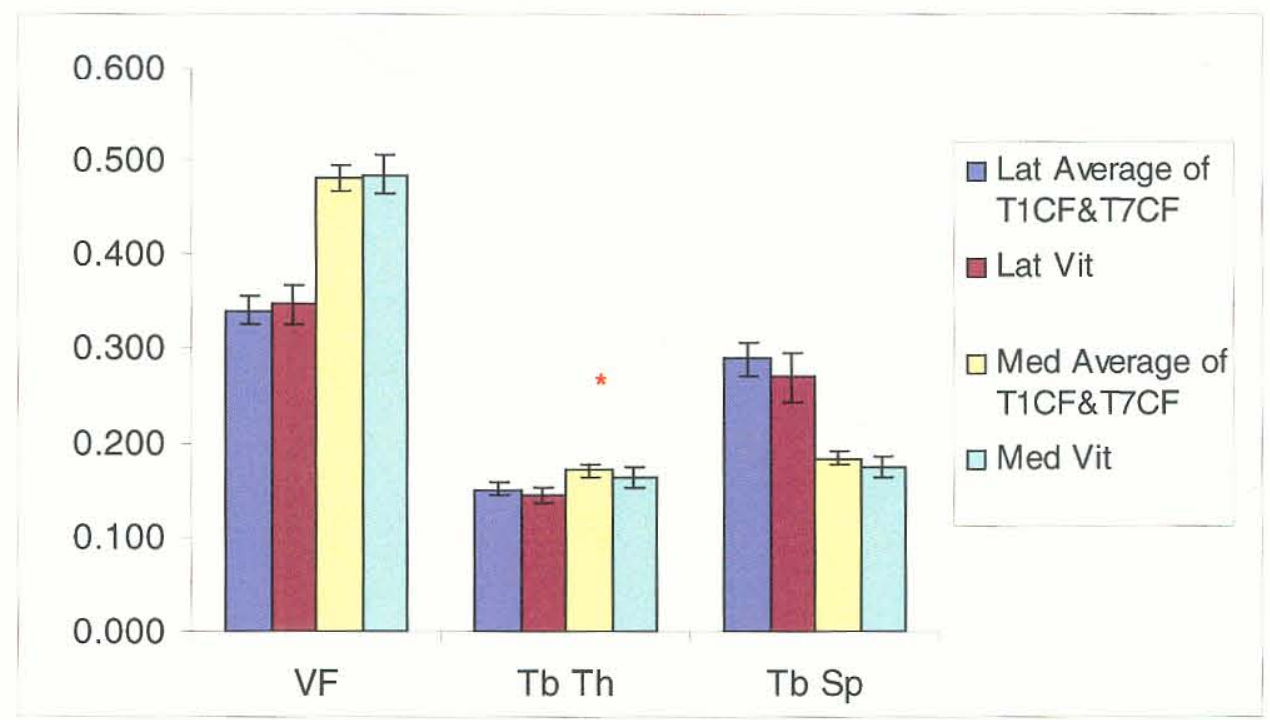

Figure 45. Lateral-medial (in vivo with correction factor-T1CF\&T7CF vs. in vitro-Vit) averages are shown with standard deviation $(\mathrm{n}=12)$ for volume fraction $(\mathrm{VF})$, trabecular thickness $(\mathrm{Tb} \mathrm{Th})$ and trabecular separation $(\mathrm{Tb} \mathrm{Sp}) .\left(^{*}=\right.$ significantly different for $\mathrm{p}<0.05$ T-Tests: paired two sample for means) The $\mathrm{y}$ - axis is unitless for VF and $\mathrm{mm}$ for $\mathrm{Tb} \mathrm{Th}$ and $\mathrm{Tb} \mathrm{Sp}$. The VF and $\mathrm{Tb} \mathrm{Sp}$ for lateral and medial condyles between T1CF\&T7CF and Vit were not significantly different $(\mathrm{p}=0.429, \mathrm{p}=0.602, \mathrm{p}=0.148$ \& $\mathrm{p}=0.107)$. The Tb Th for lateral condyles between T1CF\&T7CF and Vit were not significantly different $(\mathrm{p}=0.193)$ but significantly different for medial condyles $(\mathrm{p}=0.027)$. 
5. Archimedes, Serial Milling and Micro CT-in vitro cubes

Measurements of volume fraction (VF), trabecular thickness (Tb Th), trabecular number ( $\mathrm{Tb} \mathrm{Nm}$ ) and trabecular separation (Tb Sp) for Archimedes (Arc), Serial Milling (SM) and Micro CT (CT) in vitro cubes are given in Table 5. A total of six rabbit distal femurs, resulting in eleven medial and lateral bone cube specimens (one outlier was excluded), were used to determine the accuracy of micro CT in vitro images to Archimedes-based volume fraction as reference. 721RL was considered outlier because the average \% difference for VF between SM and Arc and between SM and CT, and for $\mathrm{Tb}$ Sp between SM and CT were not in the 95\% normal distribution curve that is between the average $\%$ difference \pm twice the standard deviation. In addition, it was an advantage that the in vitro bone cubes underwent serial milling experiment which therefore allowed the comparison of trabecular thickness, trabecular number and trabecular spacing from serial milling to micro $\mathrm{CT}$ in vitro images. The average VF for lateral and medial condyles of SM was higher than Arc and CT while the average VF for lateral and medial condyles of CT was lower than the Arc (Table 5).

The average $\mathrm{Tb} \mathrm{Th}$ and $\mathrm{Tb} \mathrm{Sp}$ for lateral and medial condyles of SM was higher than $\mathrm{CT}$ but the average $\mathrm{Tb} \mathrm{Nm}$ for lateral and medial condyles of SM was lower than CT (Table 5). Two stacks of SM and CT from the same bone cube were presented (Figure 46). The differences in volume fractions for lateral and medial condyles between SM and Arc were $0.93 \%$ and $1.94 \%$, indicating excellent precision of these directly measured volume fraction measurements. The VF for lateral and medial condyles between $\mathrm{CT}$ and Arc and between CT and SM were not significantly different $(p=0.294, p=0.649, p=$ $0.115, p=0.118$ ) (Figure 47). The overall correlation coefficient, $r$-value, for VF 
between SM and Arc, CT and Arc, and between SM and CT were 0.94, 0.91 and 0.97 respectively (Figure 48).

The $\mathrm{Tb}$ Th for lateral and medial condyles between SM and CT were significantly different ( $\mathrm{p}=0.011, \mathrm{p}=0.047$ ) but the $\mathrm{Tb} \mathrm{Sp}$ for lateral and medial condyles between SM and CT were not significantly different $(\mathrm{p}=0.181, \mathrm{p}=0.877)$ (Figure 49). Overall, the $\mathrm{Tb} \mathrm{Th}$ and $\mathrm{Tb} \mathrm{Sp}$ for SM were highly correlated with $\mathrm{CT}(\mathrm{R}=0.89,0.95)$ (Figure 50). Meanwhile, the $\mathrm{Tb} \mathrm{Nm}$ for the lateral condyles between SM and CT was not significantly different ( $p=0.207$ ) but it was significantly different for the medial condyles $(p=0.013$ ). Overall, the $\mathrm{Tb} \mathrm{Nm}$ for $\mathrm{SM}$ was also highly correlated with $\mathrm{CT}(\mathrm{R}=0.90)$. Although the $\mathrm{Tb} \mathrm{Th}$ for the lateral and medial condyles and $\mathrm{Tb} \mathrm{Nm}$ for the lateral condyles between SM and CT were significantly different but the average differences were only $9.5 \%, 5.9 \%$ and $7.7 \%$ respectively.

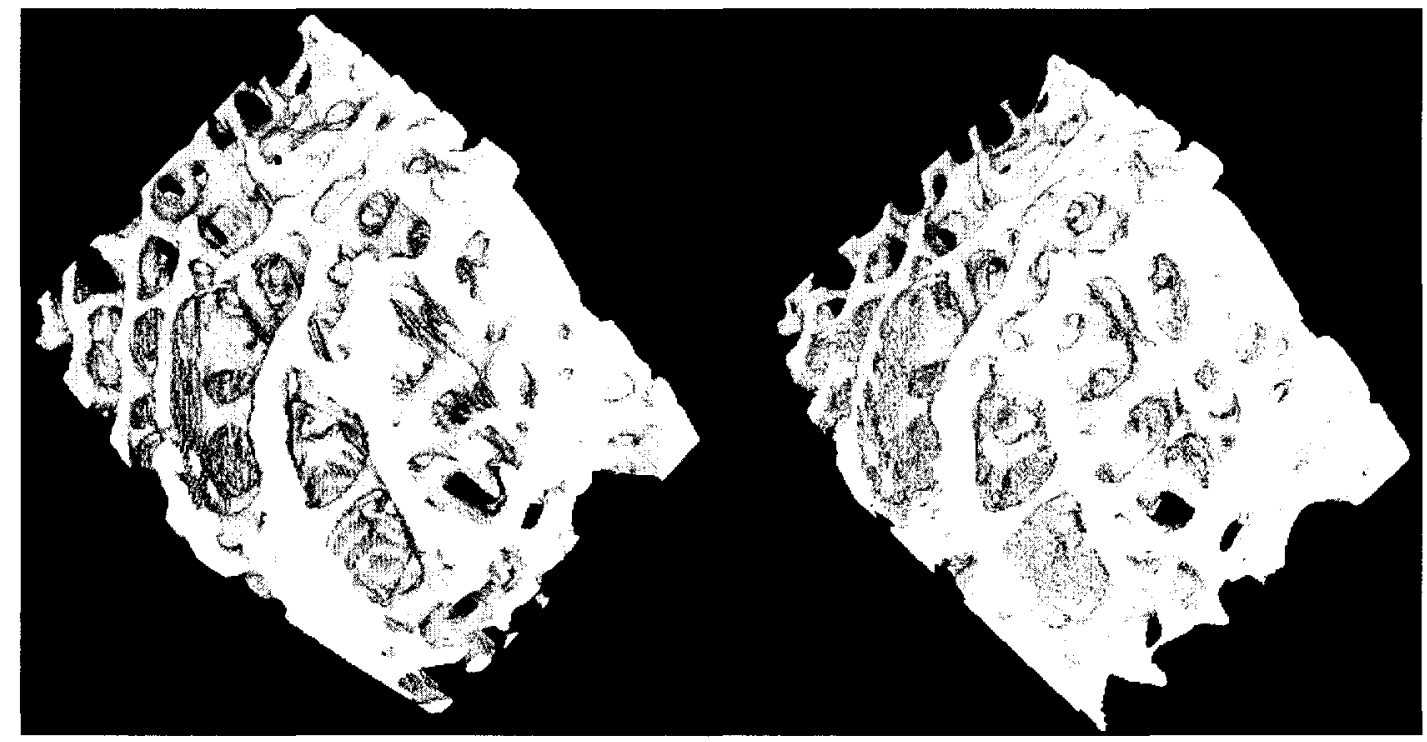

Figure 46. Three dimensional image stacks of CT (left) and SM (right) from the same bone cube after slice-by-slice global threshold. 
Table 5. Volume Fraction (VF), Trabecular Thickness (Tb Th), Trabecular Number (Tb $\mathrm{Nm}$ ) and Trabecular Spacing (Tb Sp) from Archimedes-based (arc) and serial milling (SM) experiments and in vitro micro CT (CT) scans.

\begin{tabular}{|c|c|c|c|c|c|c|}
\hline rabbits & 719RL & $720 \mathrm{RL}$ & $721 \mathrm{RL}^{\star \star}$ & $751 \mathrm{RL}$ & $718 \mathrm{LL}$ & $762 \mathrm{LL}$ \\
\hline VF (arc) & 0.318 & 0.370 & 0.328 & 0.360 & 0.371 & 0.404 \\
\hline VF (SM) & 0.316 & 0.368 & 0.387 & 0.344 & 0.394 & 0.421 \\
\hline $\mathrm{VF}(\mathrm{CT})$ & 0.316 & 0.364 & 0.310 & 0.314 & 0.387 & 0.378 \\
\hline TbTh (SM) & 0.178 & 0.190 & 0.159 & 0.197 & 0.16 & 0.190 \\
\hline TbTh (CT) & 0.163 & 0.165 & 0.141 & 0.191 & 0.146 & 0.163 \\
\hline $\mathrm{TbNm}(\mathrm{SM})$ & 1.776 & 1.934 & 2.434 & 1.750 & 2.468 & 2.221 \\
\hline $\mathrm{Tb} \mathrm{Nm}(\mathrm{CT})$ & 1.935 & 2.207 & 2.197 & 1.808 & 2.644 & 2.328 \\
\hline Tb Sp (SM) & 0.385 & 0.327 & 0.252 & 0.375 & 0.245 & 0.261 \\
\hline Tb Sp (CT) & 0.353 & 0.288 & 0.314 & 0.379 & 0.232 & 0.267 \\
\hline rabbits & 719RM & 720RM & 721RM & 751RM & 718LM & $762 \mathrm{LM}$ \\
\hline VF (arc) & 0.394 & 0.437 & 0.543 & 0.452 & 0.410 & 0.523 \\
\hline VF (SM) & 0.389 & 0.439 & 0.518 & 0.496 & 0.450 & 0.510 \\
\hline $\mathrm{VF}(\mathrm{CT})$ & 0.387 & 0.436 & 0.485 & 0.494 & 0.411 & 0.508 \\
\hline TbTh (SM) & 0.174 & 0.182 & 0.213 & 0.228 & 0.184 & 0.228 \\
\hline TbTh (CT) & 0.175 & 0.176 & 0.189 & 0.223 & 0.172 & 0.199 \\
\hline TbNm (SM) & 2.241 & 2.418 & 2.432 & 2.175 & 2.437 & 2.240 \\
\hline $\mathrm{Tb} \mathrm{Nm}(\mathrm{CT})$ & 2.207 & 2.472 & 2.572 & 2.211 & 2.397 & 2.547 \\
\hline Tb Sp (SM) & 0.273 & 0.232 & 0.198 & 0.232 & 0.226 & 0.219 \\
\hline \multirow[t]{2}{*}{ Tb Sp (CT) } & 0.278 & 0.228 & 0.200 & 0.229 & 0.246 & 0.193 \\
\hline & $\begin{array}{c}\text { Lat } \\
\text { condyles } \\
\text { AVE }\end{array}$ & $\begin{array}{c}\text { Lat } \\
\text { condyles } \\
\text { STD } \\
\end{array}$ & $\begin{array}{c}\text { Med } \\
\text { condyles } \\
\text { AVE }\end{array}$ & $\begin{array}{c}\text { Med } \\
\text { condyles } \\
\text { STD } \\
\end{array}$ & $\begin{array}{c}\text { Lat \& } \\
\text { Med } \\
\text { condyles } \\
\text { AVE }\end{array}$ & $\begin{array}{c}\text { Lat \& } \\
\text { Med } \\
\text { condyles } \\
\text { STD } \\
\end{array}$ \\
\hline VF (arc) & 0.365 & 0.031 & 0.460 & 0.060 & 0.417 & 0.068 \\
\hline VF (SM) & 0.369 & 0.041 & 0.467 & 0.050 & 0.422 & 0.068 \\
\hline $\mathrm{VF}(\mathrm{CT})$ & 0.352 & 0.035 & 0.454 & 0.049 & 0.407 & 0.067 \\
\hline TbTh (SM) & 0.183 & 0.015 & 0.202 & 0.024 & 0.193 & 0.022 \\
\hline TbTh (CT) & 0.166 & 0.016 & 0.189 & 0.020 & 0.178 & 0.021 \\
\hline TbNm (SM) & 2.030 & 0.308 & 2.324 & 0.118 & 2.190 & 0.262 \\
\hline $\mathrm{Tb} \mathrm{Nm}(\mathrm{CT})$ & 2.184 & 0.330 & 2.401 & 0.161 & 2.303 & 0.263 \\
\hline Tb Sp (SM) & 0.319 & 0.064 & 0.230 & 0.025 & 0.270 & 0.064 \\
\hline Tb Sp (CT) & 0.304 & 0.061 & 0.229 & 0.031 & 0.263 & 0.059 \\
\hline \multicolumn{7}{|c|}{$\star \star$ outlier (eliminated) } \\
\hline
\end{tabular}




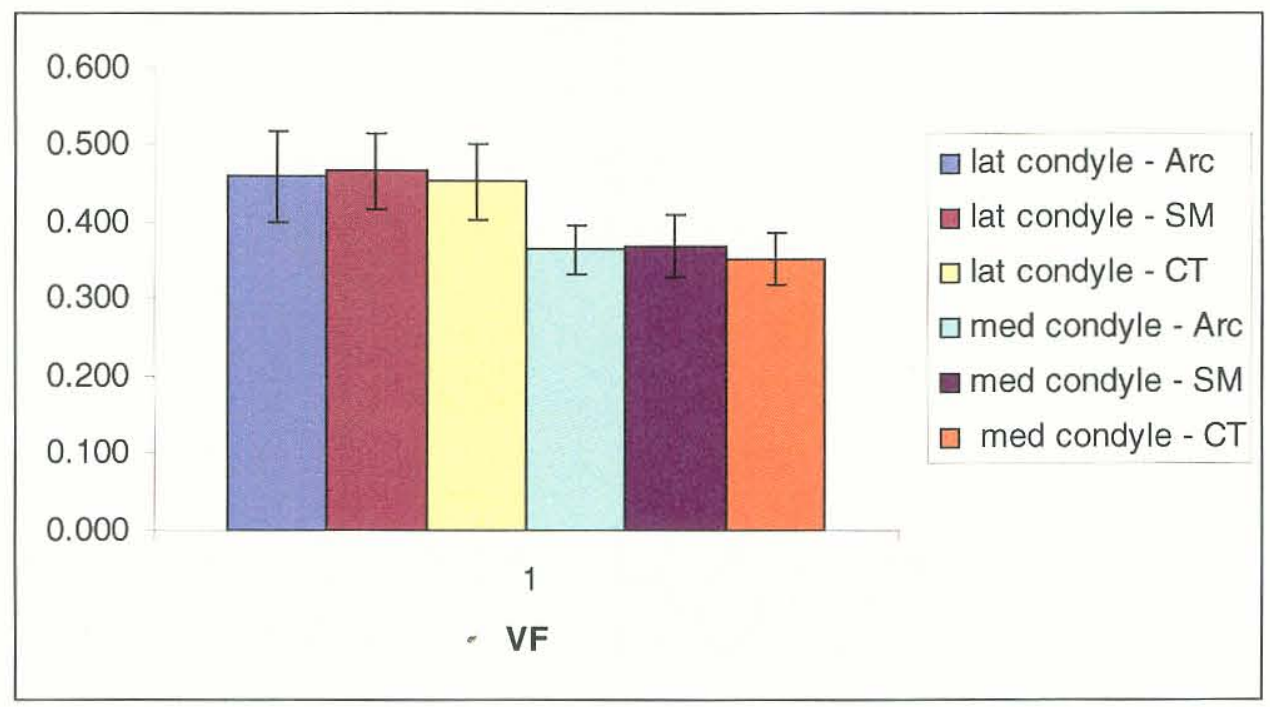

Figure 47. Average values of volume fraction (VF) for lateral and medial among Archimedes (arc), serial milling (SM) and in vitro micro CT (CT) are shown with standard deviation $(\mathrm{n}=11)$ The coefficient of variation in VF between Arc and SM ranged between $0.10 \%$ and $0.22 \%$. The VF for lateral and medial condyles between CT and Arc and between CT and SM were not significantly different $(p=0.294, p=0.649, p$ $=0.115, p=0.118)$. The $y$ - axis is unitless for VF.

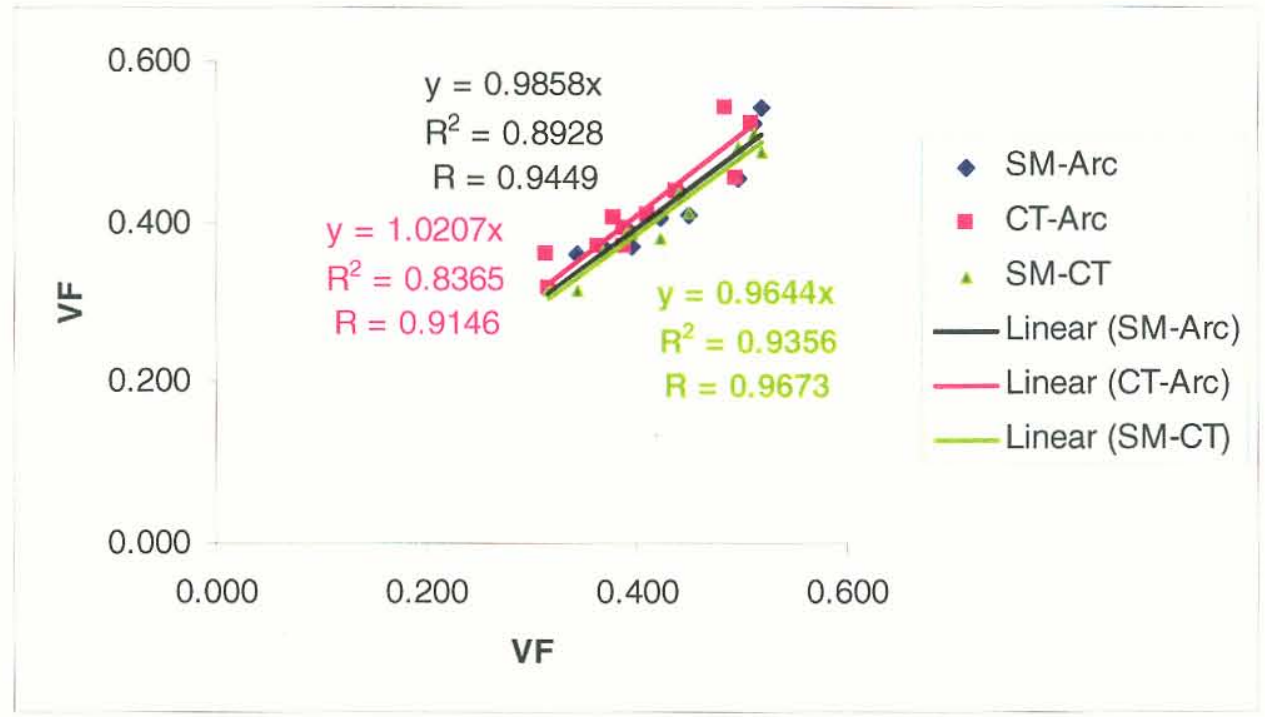

Figure 48. Correlation of volume Fraction (VF) for eleven bone cube specimens (medial and lateral condyles) in three groups (SM-arc, CT-arc \& SM-CT) were shown. The xand $y$ - axes are unitless for VF. 


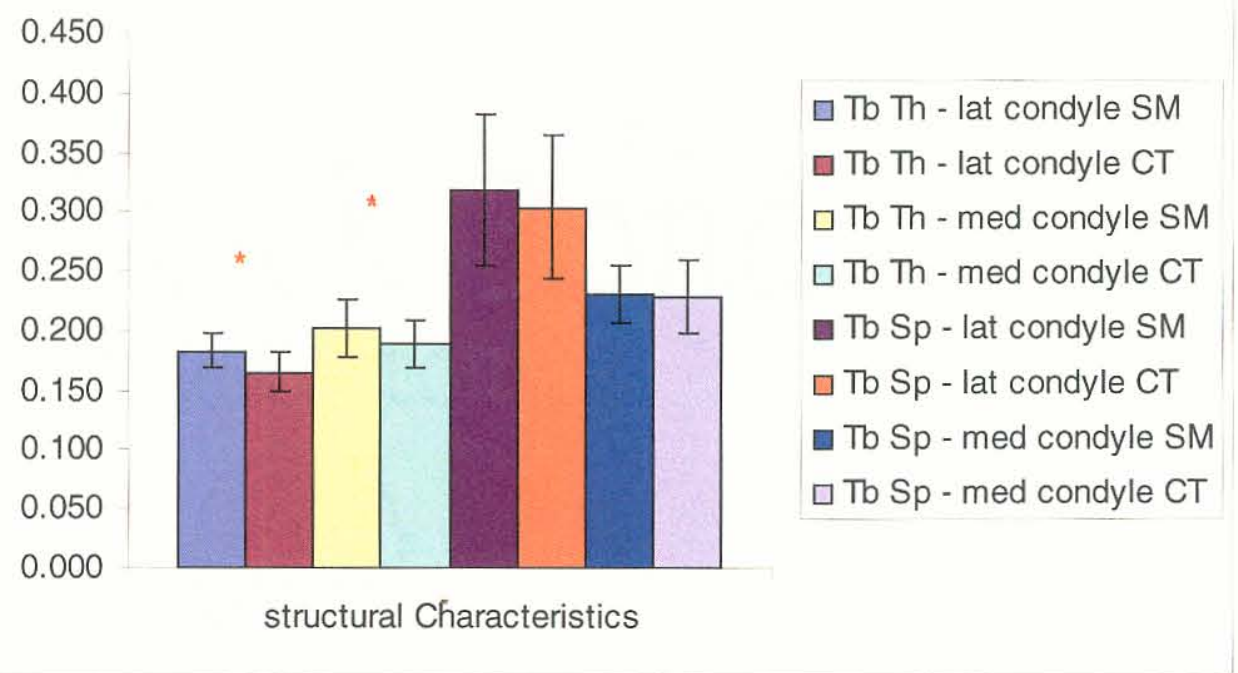

Figure 49. Trabecular Thickness ( $\mathrm{Tb} \mathrm{Th}$ ) and Trabecular Separation (Tb Sp) for lateral and medial condyles between serial milling (SM) and in vitro micro CT (CT) bone cubes specimens are shown with standard deviation $(n=11)\left(^{*}=\right.$ significantly different for p $<0.05$ t-Tests: Paired Two Sample for Means) The Tb Th for lateral and medial condyles and $\mathrm{Tb} \mathrm{Sp}$ for lateral condyles between SM and CT were significantly different $(\mathrm{p}=0.011, \mathrm{p}=0.047, \mathrm{p}=0.013$ ) but the $\mathrm{Tb} \mathrm{Sp}$ for lateral and medial condyles and $\mathrm{Tb}$ $\mathrm{Nm}$ for medial condyles between SM and CT were not significantly different $(\mathrm{p}=0.181$, $\mathrm{p}=0.877, \mathrm{p}=0.207)$. The $\mathrm{y}$ - axis is $\mathrm{mm}$ for $\mathrm{Tb} \mathrm{Th}$ and $\mathrm{Tb} \mathrm{Sp}$.

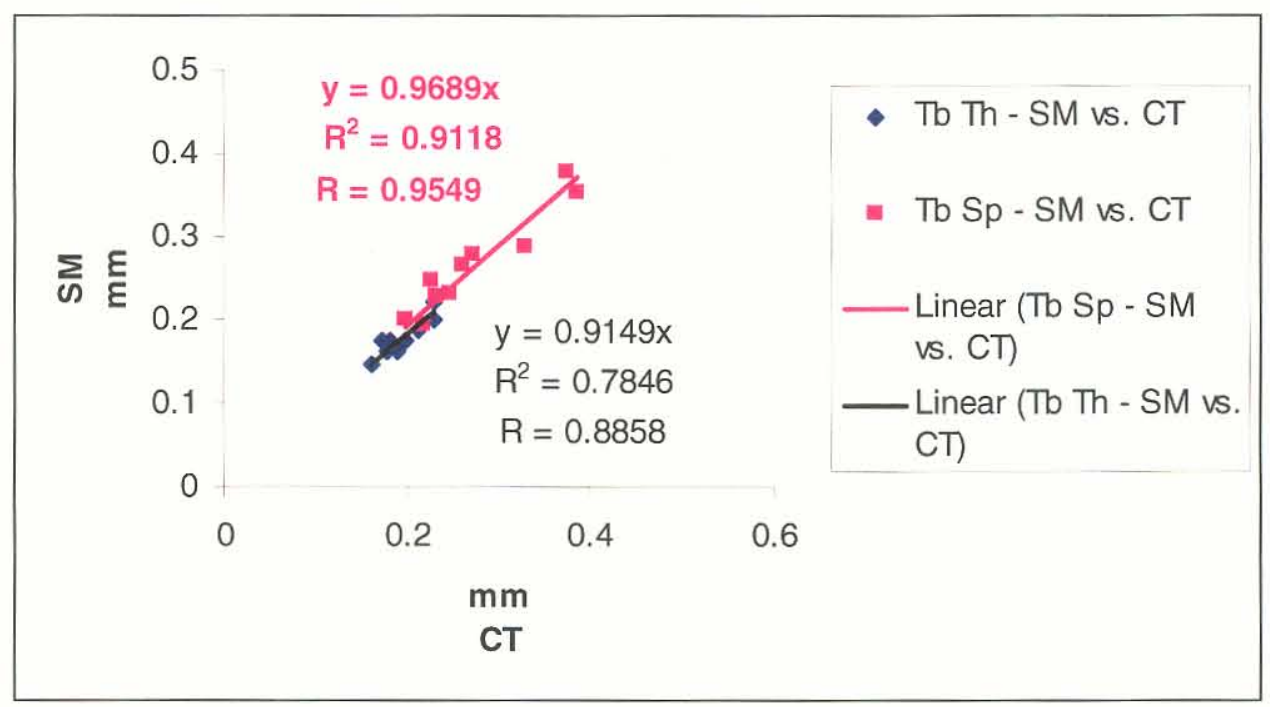

Figure 50. Correlation of trabecular thickness ( $\mathrm{Tb} \mathrm{Th}$ ) and trabecular separation ( $\mathrm{Tb} \mathrm{Sp}$ ) for eleven bone cube specimens (medial and lateral condyles) between SM and CT were reported. The $\mathrm{x}$ - and $\mathrm{y}-\mathrm{axes}$ are $\mathrm{mm}$ for $\mathrm{Tb} \mathrm{Th}$ and $\mathrm{Tb} \mathrm{Sp}$. 
6. FEA (apparent stiffness and tissue modulus) and MTS (apparent stiffness)

The FEA apparent stiffness for FEA models using 12E9 (arbitrary) tissue modulus are given in Table 6 for a total of thirteen specimens (medial and lateral) from seven rabbit distal femurs. Based on the arbitrary tissue modulus value of $12 \mathrm{E} 9$, the FEA apparent stiffness ranged from 9E8 Pa to 2.8E9 Pa giving an average apparent stiffness value of 1.9E9 $\mathrm{Pa}$ (Table 6). Only twelve specimens were analyzed and reported in Table 7 and 8, Figures 51, 54 and 55 as 718RL was eliminated. 718RL was considered outlier because the average \% difference for apparent stiffness between FEA and MTS was not in the $95 \%$ normal distribution curve that is between the average $\%$ difference \pm twice the standard deviation. The MTS apparent stiffñess values for compressive loading along the anterior-posterior anatomic axis are given in column 2, Table 7. Subsequently, all the FEA models were executed several times in order to match the MTS apparent stiffness and the FEA apparent stiffness values. The calculated tissue modulus of trabecular bone for each specimen are given in column 3 and 4, Table 7.

Table 6 FEA apparent stiffness using 12E9 (arbitrary) tissue modulus Using 12E9 (arbitrary) tissue modulus

\begin{tabular}{ll} 
& Using 12E9 (arbitrary) tissue modulus \\
\hline Rabbit bone & FEA apparent stiffness (Pa) \\
\hline $721 \mathrm{LL}$ & $1.11 \mathrm{E}+09$ \\
$721 \mathrm{LM}$ & $1.99 \mathrm{E}+09$ \\
$720 \mathrm{LL}$ & $2.71 \mathrm{E}+09$ \\
$720 \mathrm{LM}$ & $1.84 \mathrm{E}+09$ \\
$718 \mathrm{RL} *$ & $1.60 \mathrm{E}+09$ \\
$718 \mathrm{RM}$ & $2.61 \mathrm{E}+09$ \\
$762 \mathrm{RL}$ & $1.22 \mathrm{E}+09$ \\
762RM & $2.45 \mathrm{E}+09$ \\
751LL & $1.40 \mathrm{E}+09$ \\
751LM & $2.82 \mathrm{E}+09$ \\
750RL & $9.35 \mathrm{E}+08$ \\
750RM & $2.62 \mathrm{E}+09$ \\
719LL & $1.22 \mathrm{E}+09$ \\
\hline AVERAGE & $1.89 \mathrm{E}+09$ \\
\hline STDEV & $6.89 \mathrm{E}+08$ \\
\hline
\end{tabular}


Table 7. Tissue Modulus values of FEA based on MTS apparent stiffness

\begin{tabular}{|c|c|c|c|}
\hline & MTS & FEA & Tissue \\
\hline Rabbit bone & $\begin{array}{l}\text { App. Stiffness } \\
\text { (Pa) }\end{array}$ & $\begin{array}{l}\text { App. Stiffness match with MTS } \\
\text { (Pa) }\end{array}$ & $\begin{array}{l}\text { Modulus } \\
(\mathrm{Pa})\end{array}$ \\
\hline $721 \mathrm{LL}$ & $1.23 \mathrm{E}+09$ & $1.23 \mathrm{E}+09$ & $1.18 E+10$ \\
\hline 721LM & $1.30 \mathrm{E}+09$ & $1.33 \mathrm{E}+09$ & $8.00 E+09$ \\
\hline $720 \mathrm{LL}$ & $2.72 \mathrm{E}+09$ & $2.71 E+09$ & $1.20 \mathrm{E}+10$ \\
\hline $720 \mathrm{LM}$ & $1.16 \mathrm{E}+09$ & $1.16 E+09$ & $7.60 \mathrm{E}+09$ \\
\hline 718RM & $2.84 \mathrm{E}+09$ & $2.85 E+09$ & $1.31 E+10$ \\
\hline 762RL & $1.04 \mathrm{E}+09$ & $1.06 \mathrm{E}+09$ & $1.04 \mathrm{E}+10$ \\
\hline 762RM & $2.23 E+09$ & $2.25 \mathrm{E}+09$ & $1.10 \mathrm{E}+10$ \\
\hline $751 \mathrm{LL}$ & $1.30 \mathrm{E}+09$ & $1.32 \mathrm{E}+09$ & $1.13 E+10$ \\
\hline 751LM & $2.82 E+09$ & $2.82 E+09$ & $1.20 \mathrm{E}+10$ \\
\hline 750RL & $1.11 \mathrm{E}+09$ & $1.09 \mathrm{E}+09$ & $1.40 E+10$ \\
\hline $750 \mathrm{RM}$ & $2.15 E+09$ & $2.19 \mathrm{E}+09$ & $1.00 \mathrm{E}+10$ \\
\hline 719LL & 1.17E+09 & $1.18 \mathrm{E}+09$ & $1.16 \mathrm{E}+10$ \\
\hline average & $1.76 \mathrm{E}+09$ & $1.77 \mathrm{E}+09$ & $1.11 \mathrm{E}+10$ \\
\hline stdev & $7.37 \mathrm{E}+08$ & $7.35 \mathrm{E}+08$ & $1.87 \mathrm{E}+09$ \\
\hline 718RL** (eliminated) & $9.34 \mathrm{E}+08$ & $9.32 E+08$ & $7.00 E+09$ \\
\hline
\end{tabular}

Using the average tissue modulus of 11.1E9 $\mathrm{Pa}$ on each FEA model, the FEA apparent stiffness values for each bone cube specimen was recalculated and they ranged from 0.6 to $2.53 \mathrm{GPa}$ (Table 8 ). An example of the force distribution of an FEA model is presented in Figure 51. The average difference for apparent stiffness between FEA and experimental (MTS) was $15.84 \%$ however, they were not significantly different $(\mathrm{p}=$ 0.7324). The $r$ value for apparent stiffness between FEA (using average tissue modulus of $11.1 \mathrm{GPa}$ ) and MTS was 0.8721 (Figure 52). 
Table 8. Comparison of Apparent Stiffness Values from MTS and FEA (using average tissue modulus)

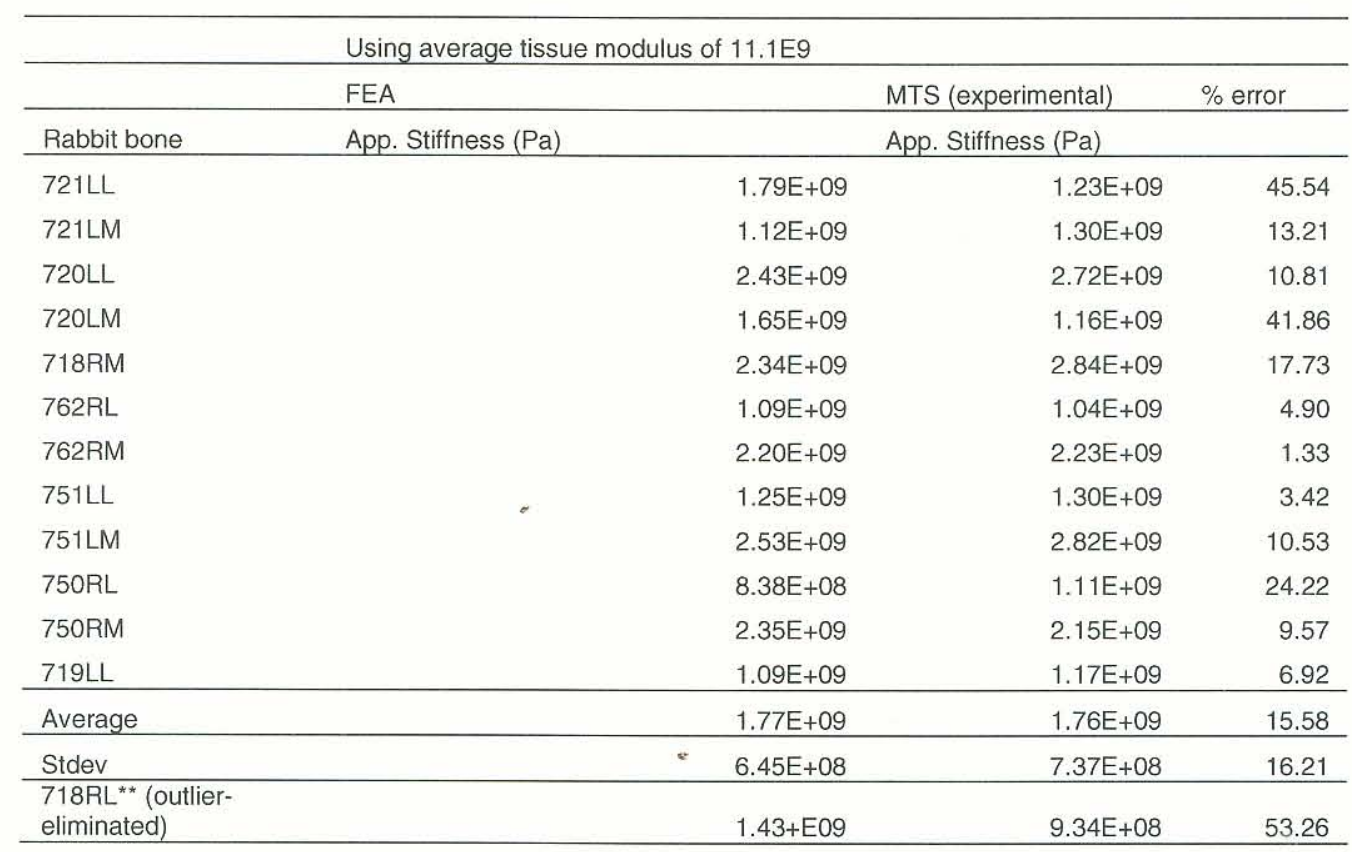

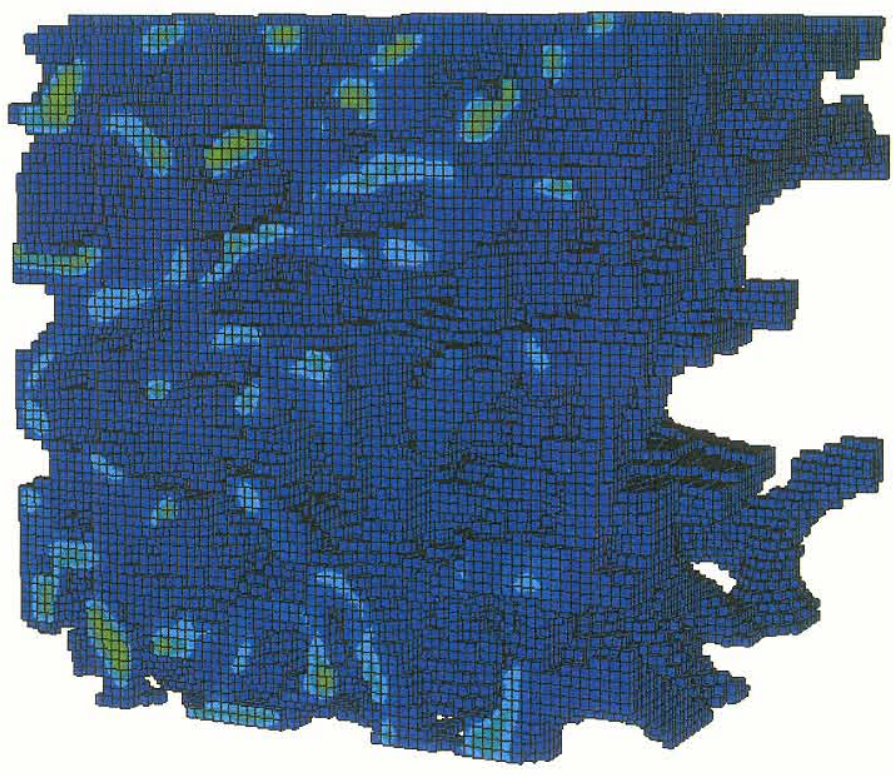

Figure 51. Force distribution of an FEA model 


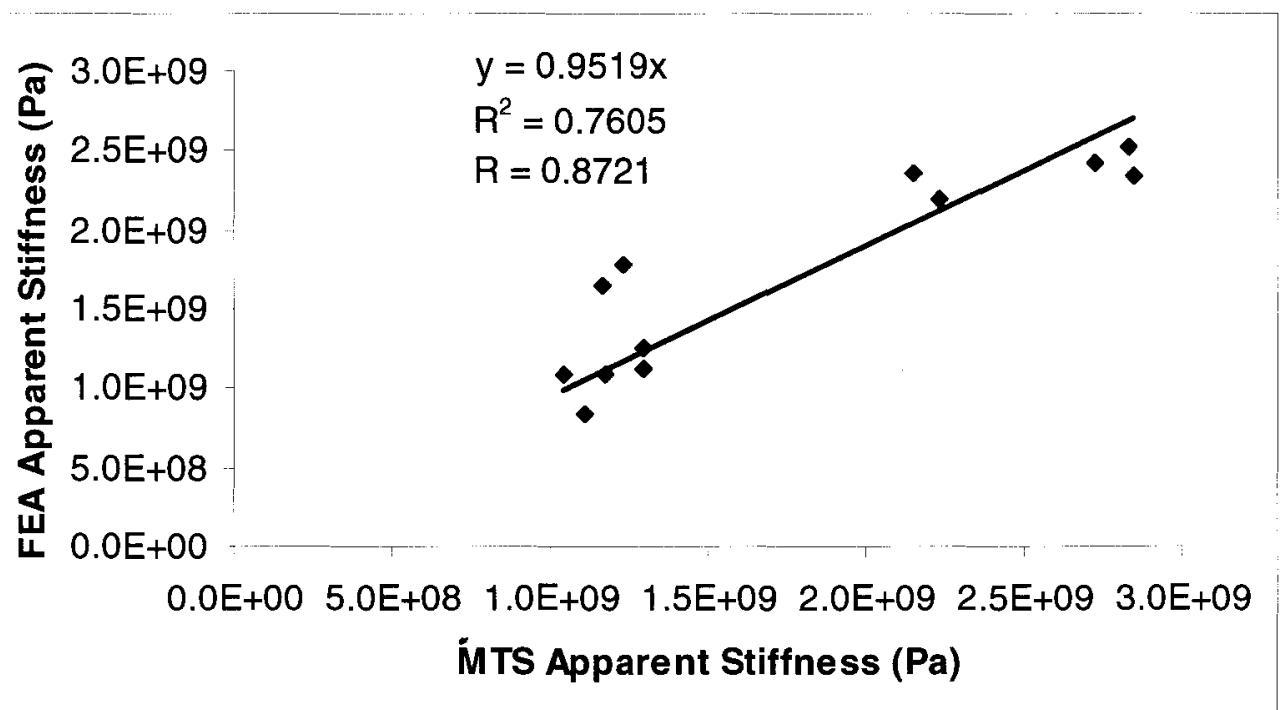

Figure 52. Apparent Stiffness of twelve rabbit distal femur bone cube specimens (medial and lateral condyles) measured in nondestructive mechanical testing (FEA) were plotted against mechanical testing experiment (MT\$).

In addition to the apparent stiffness from mechanical compressive testing (MTS) and finite element analysis based on tissue modulus of $11.1 \mathrm{GPa}$, volume fraction (VF), trabecular thickness $(\mathrm{Tb} \mathrm{Th})$, trabecular number $(\mathrm{Tb} \mathrm{Nm})$ and trabecular separation $(\mathrm{Tb}$ $\mathrm{Sp}$ ) of the medial and lateral condyles for a total of twelve bone cube specimens are given in Table 9. It should be noted that two specimens (721LL \& 720LM)-with the lowest volume fraction in the lateral and medial groups respectively-had percentage errors of $45.54 \%$ and $41.86 \%$ respectively; all other errors for lateral and medial groups were in the range of $4 \%$ to $24 \%$ and $1 \%$ to $18 \%$, respectively. Only the volume fraction and the trabecular spacing of the bone cube specimens are correlated with the FEA apparent stiffness ( $R=0.84$ and $R=0.71$, respectively) (Figure 53). The $R^{2}$ value or coefficient of determination of 0.70 for the volume fraction demonstrated the variation between FEA apparent stiffness and volume fraction was within $70 \%$, while the variations between FEA and trabecular spacing were within 50\% (Figure 53). Meanwhile, the apparent 
stiffness values have low correlation with the trabecular thickness or trabecular number (Figure 53). The FEA apparent stiffness values have correlation to the structural characteristics that was similar to the experimental methods (Figure 54). Additionally, the FEA apparent stiffness is correlated with $\mathrm{Tb} \mathrm{Nm}$ with an $\mathrm{r}$ value of 0.47 which was close to the $\mathrm{r}$ value of 0.52 for the correlation of the MTS apparent stiffness and $\mathrm{Tb} \mathrm{Nm}$.

Table 9. VF, $\mathrm{Tb} \mathrm{Th}, \mathrm{Tb} \mathrm{Nm}$ and $\mathrm{Tb} \mathrm{Sp}$ of the bone cube specimens (medial and lateral condyles) for mechanical compressive testing (MTS) and finite element analysis (FEA).

\begin{tabular}{|c|c|c|c|c|c|c|c|}
\hline $\begin{array}{l}\text { Rabbit } \\
\text { bone }\end{array}$ & MTS & FEA & VF & $\mathrm{Tb}$ Th & Tb Nm & Tb Sp & $\%$ error \\
\hline Lateral & $\begin{array}{l}\text { App. } \\
\text { Stiffness } \\
\mathrm{Pa}\end{array}$ & $\begin{array}{l}\text { App. } \\
\text { Stiffness } \\
\mathrm{Pa}\end{array}$ & & " $\mathrm{mm}$ & $\mathrm{mm}^{-1}$ & $\mathrm{~mm}$ & $\begin{array}{l}\text { Absolute } \\
\text { value }\end{array}$ \\
\hline 721LL & $1.23 \mathrm{E}+09$ & $1.79 \mathrm{E}+09$ & 0.305 & 0.150 & 2.037 & 0.341 & 45.54 \\
\hline 720LL & 2.72E+09 & $2.43 \mathrm{E}+09$ & 0.437 & 0.185 & 2.364 & 0.238 & 10.81 \\
\hline 762RL & $1.04 \mathrm{E}+09$ & $1.09 \mathrm{E}+09$ & 0.309 & 0.138 & 2.234 & 0.309 & 4.90 \\
\hline 751LL & $1.30 E+09$ & $1.25 E+09$ & 0.358 & 0.216 & 1.657 & 0.388 & 3.42 \\
\hline 750RL & $1.11 \mathrm{E}+09$ & $8.38 E+08$ & 0.290 & 0.146 & 1.991 & 0.357 & 24.22 \\
\hline $719 \mathrm{LL}$ & 1.17E+09 & $1.09 \mathrm{E}+09$ & 0.307 & 0.149 & 2.064 & 0.336 & 6.92 \\
\hline AVE & $1.43 E+09$ & 1.41E+09 & 0.334 & 0.164 & 2.058 & 0.328 & 15.97 \\
\hline STDEV & $6.41 \mathrm{E}+08$ & $5.90 \mathrm{E}+08$ & 0.055 & 0.030 & 0.241 & 0.051 & 16.31 \\
\hline & MTS & FEA & VF & Tb Th & $\mathrm{Tb} \mathrm{Nm}$ & Tb Sp & $\%$ error \\
\hline Medial & $\begin{array}{l}\text { App. } \\
\text { Stiffness } \\
\mathrm{Pa}\end{array}$ & $\begin{array}{l}\text { App. } \\
\text { Stiffness } \\
\mathrm{Pa}\end{array}$ & & $\mathrm{mm}$ & $\mathrm{mm}^{-1}$ & $\mathrm{~mm}$ & $\begin{array}{l}\text { Absolute } \\
\text { value }\end{array}$ \\
\hline 721LM & $1.30 \mathrm{E}+09$ & $1.12 \mathrm{E}+09$ & 0.392 & 0.159 & 2.466 & 0.247 & 13.21 \\
\hline 720LM & $1.16 \mathrm{E}+09$ & $1.65 \mathrm{E}+09$ & 0.345 & 0.178 & 1.942 & 0.337 & 41.86 \\
\hline 718RM & 2.84E+09 & 2.34E+09 & 0.425 & 0.162 & 2.619 & 0.220 & 17.73 \\
\hline 762RM & 2.23E+09 & $2.20 \mathrm{E}+09$ & 0.423 & 0.168 & 2.514 & 0.229 & 1.33 \\
\hline 751LM & $2.82 \mathrm{E}+09$ & $2.53 E+09$ & 0.451 & 0.207 & 2.181 & 0.252 & 10.53 \\
\hline 750RM & $2.15 E+09$ & $2.35 \mathrm{E}+09$ & 0.442 & 0.193 & 2.294 & 0.243 & 9.57 \\
\hline AVE & $2.08 \mathrm{E}+09$ & $2.03 E+09$ & 0.413 & 0.178 & 2.336 & 0.255 & 15.71 \\
\hline STDEV & 7.24E+08 & $5.37 \mathrm{E}+08$ & 0.039 & 0.019 & 0.249 & 0.042 & 13.90 \\
\hline
\end{tabular}




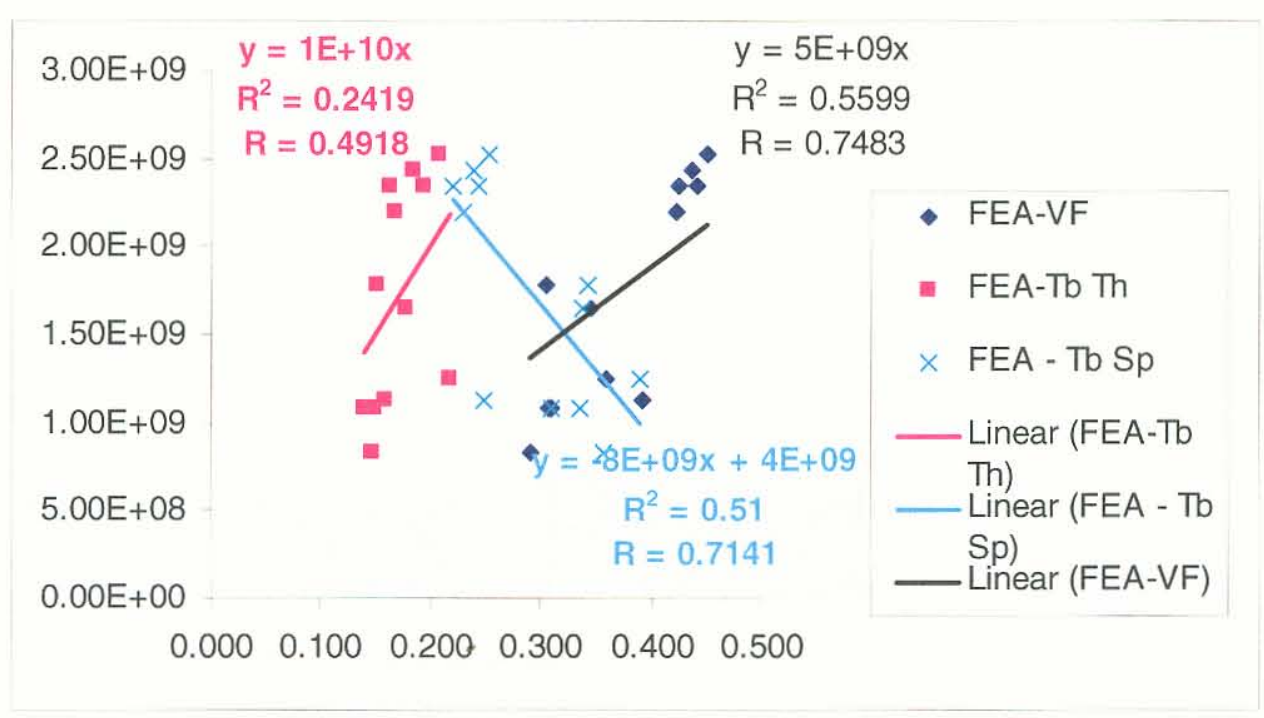

Figure 53. FEA Apparent stiffness of twelve rabbit distal femur bone cube specimens (medial and lateral condyles) were plotted against volume fraction (VF), trabecular thickness ( $\mathrm{Tb} \mathrm{Th}$ ), and trabecular separation $(\mathrm{Tb} \mathrm{Sp})$. The $\mathrm{x}$-axis is $\mathrm{Pa}$ for apparent stiffness while the $\mathrm{y}$-axis is unitless for VF, $\mathrm{mm}$ for $\mathrm{Tb} \mathrm{Th}$ and $\mathrm{Tb} \mathrm{Sp}$.

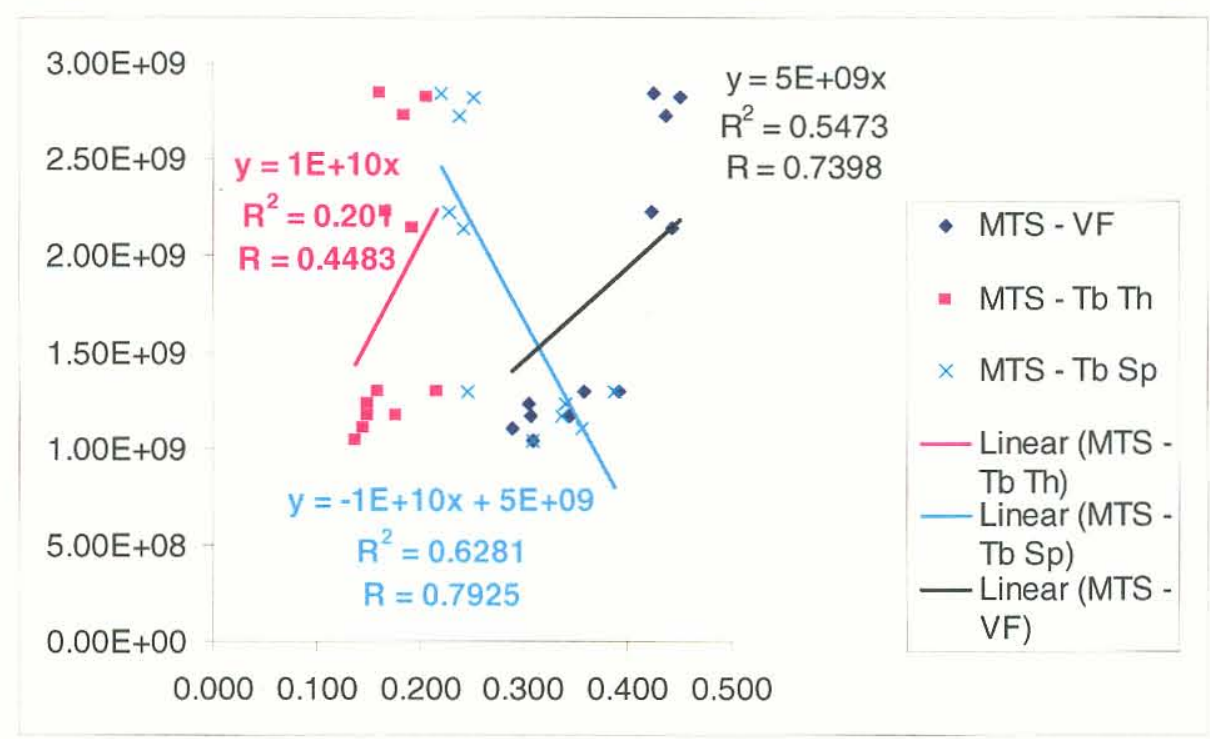

Figure 54. MTS Apparent stiffness of twelve rabbit distal femur bone cube specimens (medial and lateral condyles) were plotted against volume fraction (VF), trabecular thickness ( $\mathrm{Tb} \mathrm{Th}$ ), and trabecular separation (Tb Sp). The $\mathrm{x}$-axis is $\mathrm{Pa}$ for apparent stiffness while the $\mathrm{y}$-axis is unitless for VF, $\mathrm{mm}$ for Tb Th and $\mathrm{Tb} \mathrm{Sp}$. 


\section{DISCUSSION}

The volume fraction, trabecular thickness and trabecular number for $\mathrm{T} 1$ and $\mathrm{T} 7$ for the medial condyles were higher than lateral condyles indicating that the medial condyles are denser than lateral condyles (Table 1). The VF and other trabecular structures measurements between $\mathrm{T} 1$ and $\mathrm{T} 7$ were expected to be in excellent agreement as the average percentage difference between these two in vivo scans was within $1.7 \%$. This shows that the micro CT scanning is consistently repeatable. The volume fraction, trabecular thickness and trabecular number for $\mathrm{T} 7{ }^{\prime}$ and $\mathrm{Vit}$ of the medial condyles were also higher than the lateral condyles (Table 2) just like the previous comparison between medial and lateral condyles of $\mathrm{T} 1$ and $\mathrm{T} 7$. In addition, the volume fraction and other structural characteristics measurements for medial and lateral condyles of $\mathrm{T} 7$ ' were higher than Vit (Table 2) indicating an over estimation of $28 \mu \mathrm{m}$ in vivo scans to $14 \mu \mathrm{m}$ in vitro scans.

Meanwhile, the \% difference between T7 and Vit for the lateral condyles is twice the $\%$ difference between $\mathrm{T}^{\prime}$ and Vit for the medial condyle indicating that a less dense bone could lead to higher over estimation between in vivo and in vitro scans. This also suggests that a high resolution $(\leq 28 \mu \mathrm{m})$ micro CT scanning may be necessary when dealing with low density bone. Although the $14 \mu \mathrm{m}$ in vitro scans were degraded to $28 \mu \mathrm{m}$ for the purpose of statistical analysis and comparison, the quality of the scans still remain 
high, giving a better prediction of actual bone volume and structural characteristics of trabecular bone. This is because there is more bone information captured in a $14 \mu \mathrm{m}$ image than a $28 \mu \mathrm{m}$ image. The average volume fraction measurements for medial and lateral condyles from $14 \mu \mathrm{m}$ in vitro scans of 0.485 and 0.348 were compared to the results of 0.48 and 0.39 for the medial and lateral condyles based on five mature New Zealand white rabbits reported by Bourne et al, 2002 respectively. This comparison showed two important capabilities; (1) the reliability and consistency of the slice-by-slice global thresholding technique and (2) our expectation of believing that the quality of $14 \mu \mathrm{m}$ in vitro micro CT scans, which is reliable, is correct. The accuracy of our $14 \mu \mathrm{m}$ in vitro micro CT scan is also confirmed by the excellent agreement between our scan data and the Archimedes'-based data. For in vivo scanning, the addition of a percentage correction factor resulted in an acceptable bone structural characteristics measurement at $28 \mu \mathrm{m}$ resolution (Table 6). The average correction factor of $1 \%$ and $0.5 \%$ (Table 6) can be applied to lateral and medial condyles respectively, of in vivo scans giving measurements of volume fraction and structural characteristics which are similar to the $14 \mu \mathrm{m}$ in vitro scans.

The results between the in vitro micro CT cubes and Archimedes-based volume fractions showed $2.5 \%$ underestimation. The underestimation of micro CT scanning was expected due to the partial volume effects from the slice-by-slice global thresholding technique. However, this percentage is well within the range reported by Ding et al. 1999 that the micro CT scanning was underestimating the volume fraction by $4 \%$ compared to Archimedes. Meanwhile, the results between the serial milling and Archimedes-based volume fractions showed $2.5 \%$ overestimation. The overestimation from serial milling 
was due to the residue of silver nitrate adjacent to the trabecular bone. However, percent error from this study is well within 5\% reported by Beck et al. 1997.

The overall in vitro micro CT (CT) VF, $\mathrm{Tb} \mathrm{Th}$ and $\mathrm{Tb} \mathrm{Nm}$ measurements in this study $(0.407 \pm 0.067,0.178 \pm 0.021,2.303 \pm 0.263)$ were also compared to the overall results $(0.226 \pm 0.006,0.167 \pm 0.032,1.63 \pm 0.22)$, which based on human proximal tibia specimens that were micro CT scanned at $22 \mu \mathrm{m}$, reported by Ding \& Hvid et al. 2000. The Tb Th of rabbit distal femur bone specimens is only $0.011 \mathrm{~mm}$ thicker than the $\mathrm{Tb} \mathrm{Th}$ of human proximal tibia bone specimens showing that the $\mathrm{Tb}$ Th reported in this study is within an acceptable range. This is due to different species and different anatomic locations. Furthermore, the standard deviation of 0.021 and 0.2 for $\mathrm{Tb} \mathrm{Th}$ and $\mathrm{Tb} \mathrm{Nm}$ reported in this study is well within the range of 0.032 and 0.22 respectively. The only explanation for having a volume fraction value of 0.407 with a $\mathrm{Tb}$ Th value of 0.178 which is close to $\mathrm{Tb}$ Th value of 0.167 that has a volume fraction of 0.226 is the increase in the $\mathrm{Tb} \mathrm{Nm}$. The overall in vitro micro CT results are in excellent agreement for this explanation.

According to Kothari et al. 1998, it was reported VF of $\sim 0.2$, Tb $\mathrm{Nm}$ of $\sim 2.5$, Tb $\mathrm{Th}$ of $\sim 0.09$ and $\mathrm{Tb} \mathrm{Sp}$ of $\sim 0.5$ for human trabecular bone from femur and $\mathrm{VF}$ of $\sim 0.05, \mathrm{~Tb}$ $\mathrm{Nm}$ of $\sim 0.5$, Tb Th of $\sim 0.08$ and $\mathrm{Tb} \mathrm{Sp}$ of $\sim 1.9$ for human trabecular bone from vertebral bodies at a slice thickness of $100 \mu \mathrm{m}$. These specimens were serially milled and imaged at a resolution of $40 \mu \mathrm{m}$ and then artificially degraded to an in-plane resolution of $100 \mu \mathrm{m}$ and an out-of-plane (slice) resolution of 100-1000 $\mu \mathrm{m}$. Meanwhile, another study of pig tibia trabecular bones by Tanck et al. 2001 reported an average VF of 0.28 at age 104 weeks. Comparing all these values to the overall average, both medial and lateral condyles, in vitro micro CT (CT) measurements of VF $(\sim 0.407 \pm 0.067), \mathrm{Tb} \mathrm{Sp}(\sim 0.263 \pm$ 
0.059), $\mathrm{Tb} \mathrm{Th}(\sim 0.178 \pm 0.021)$ and $\mathrm{Tb} \mathrm{Nm}(\sim 2.303 \pm 0.263)$ in this study, the measurements reported in this study were apparently higher. This is due to different anatomic locations as well as different species, but not unexpected.

The overall average apparent stiffness of $1.76 \mathrm{GPa} \pm 7.37 \mathrm{E} 8 \mathrm{~Pa}$ from mechanical compressive testing using platens for 12 rabbit distal femur bone specimens in this study yielding a $40 \%$ variation was well within a $42 \%$ variation from a study (Keaveny et al. 1997) of 17 bovine tibia bones specimens reporting the average apparent stiffness of 1.94GPa $\pm 8.2 \mathrm{E} 8 \mathrm{~Pa}$ from mechanical compressive platens testing. Additionally, the average apparent stiffness for medial and lateral condyles of $2.08 \mathrm{GPa} \pm 7.24 \mathrm{E} 8 \mathrm{~Pa}$ and $1.43 \mathrm{GPa} \pm 6.41 \mathrm{E} 8 \mathrm{~Pa}$ respectively were not far off although slightly lower than the average apparent stiffness for medial and lateral condyles New Zealand white rabbit distal femur of approximately $2.5 \mathrm{GPa}$ and $1.8 \mathrm{GPa}$ respectively reported by Bourne et al. 2002 . The apparent stiffness between FEA and MTS (mechanical compressive testing) was not significantly different indicating that our FEA models are able to predict the apparent stiffness of trabecular bone within an accuracy of better than $15 \%$ error. This indicates that the method of direct conversion technique applied to micro-CT images which translates all the bone information into three dimensional models is valid. In addition, the apparent stiffness values between MTS and FEA are highly correlated indicating the slice-by-slice global thresholding technique is consistent and reliable.

The average value tissue modulus of $11.1 \mathrm{GPa} \pm 2.11 \mathrm{GPa}$ obtained from the range of correct tissue modulus values for the twelve bone specimens was well within the reported trabecular bone tissue modulus of 0.76 to $20 \mathrm{GPa}$ which based on different anatomic locations and species (vertebra, iliac crest, femur and tibia from human and bovine) (Guo, 
2001) as well as the reported trabecular bone tissue modulus of 8.1-24.3 GPa which based on ten medial and lateral femoral condyles of skeletally mature New Zealand White rabbits (Bourne et al. 2004). The standard deviation from the average tissue modulus of $11.1 \mathrm{GPa} \pm 2.11 \mathrm{GPa}$ in this study yielded a $19 \%$ variation. Meanwhile, the standard deviations from the average tissue modulus of $10.4 \mathrm{GPa} \pm 3.5 \mathrm{GPa}$ and 14.8GPa $1.4 \mathrm{GPa}$ from tensile test (dry) and ultrasonic test of human tibia trabecular bone (Rho et al. 1993), 10.9GPa $\pm 1.6 \mathrm{GPa}$ and $12.7 \mathrm{GPa} \pm 2.0 \mathrm{GPa}$ from ultrasonic test of bovine femur and human femur trabecular bone (Ashman et al. 1988), and 17.3GPa $\pm 2.62 \mathrm{GPa}$ from serial-sectioning-based finite element models of bovine tibia (Niebur et al. 1999) yielded $33 \%, 10 \%, 15 \%, 16 \%$ and $15 \%$ variations respectively. This shows that the standard deviation reported from the average tissue modulus of trabecular bone in this study was well within the accepted range of standard deviation reported in the literature. 


\section{CONCLUSIONS}

The results in this study have demonstrated the reliability, sensitivity and consistency of the slice-by-slice global thresholding technique on segmentation with and without a percentage correction factor to the in vivo and in vitro micro CT images, respectively. Our work indicates that the segmentation technique developed and applied on micro CT images yields results that are statistically comparable to those obtained from density measurement based on Archimedes' principle and serial milling experiments with intact bones tested in vitro. Having our initial study of trabecular micro architecture measurements from in vivo and in vitro micro CT scans validated by these experimental techniques, proved the repeatability of the high resolution micro CT scanning can be consistently achievable.

To further use the micro CT images in non-destructive mechanical analyses to correlate the effects of mechanical properties and structural characteristics of trabecular bone, necessitated the development of a direct conversion technique for translating micro CT images into three dimensional models. The results from this part of experiment (FEA vs. mechanical compressive testing (MTS)) also indicated the validity and capability of the direct conversion technique applied to all the micro CT images into FEA models is consistently and reliably achievable. This part of the work yields results that are statistically comparable to those obtained from destructive mechanical compressive testing, with intact bones tested in vitro. The determination of a correct tissue modulus in each bone cube specimen and the insignificant differences between FEA and MTS 
apparent stiffness values implies that the non destructive mechanical compressive analysis using FEA can provide reliable results. The apparent stiffness, an indirect but highly correlated measurement of bone strength, can be used in comparison to structural characteristics of trabecular bone for future in vivo analyses of trabecular micro architecture changes.

In conclusion, the objective of this dissertation has been accomplished by the verification of the hypotheses of this dissertation: (1) high resolution micro CT can be used to investigate the trabecular micro structural changes within a living animal within an accuracy of $2.5 \%$ and (2) micro finite element analysis can be used as a nondestructive analysis to determine the apparent stiffness, an indirect measurement of bone strength, with a correlation of $87 \%\left(\mathrm{R}^{2}=0.76\right)$. Hence, trabecular bone quality can be reasonably measured in the form of apparent stiffness within a living rabbit. 


\section{REFERENCES}

Arnold, J.S. (1960). Quantification of mineralization of bone as an organ and tissue in osteoporosis. Clin. Orthop. Rel. Res. 17, 167-175.

Ashby, M.F. (1983). The mechanical properties of cellular solids. Metallurgy Transactions 14A, 1755-1769.

Ashman, R.B. \& Rho, J.Y. (1988). Elastic modulus of trabecular bone material. Journal of Biomechanics 21, 177.

Beck, J.D., Canfield, B.L., Haddock, S.M., Chen T.J.H., Kothari, M. \& Keaveny, T.M. (1997) Three-dimensional imaging of trabecular bone using the computer numerically controlled milling technique. Bone 21(3), 281-287.

Brown, T.D. \& Ferguson, A.B. (1980). Mechanical property distributions in the cancellous bone of the human proximal femur. Acta Orthop. Scand. 51, 429-437.

Bonse, U., Busch, F., Günnewig, O., Beckmann, F., Pahl, R., Delling, G., Hahn, M. \& Graeff, W. (1994). 3D computed X-ray tomography of human cancellous bone at 8- $\mu \mathrm{m}$ spatial and $10^{-4}$ energy resolution. Bone Mineral 25, 25-38.

Carter, D.R. \& Hayes, W.C. (1977). The compressive behaviour of bone as a two-phase porous structure. J. Bone Jt. Surg. 59A, 954-962.

Bourne, B.C., Morgan, T.G., Paschalis, E.P. \& Van Der Meullen, M.C. (2002). Cancellous bone anisotropy arises from both architecture and material properties. $48^{\text {th }}$ Annual Meeting of the Orthopaedic Research Society, 558.

Bourne, B.C., Marjolein, C.H., \& Meulen, V.D. (2004). Finite element models predict cancellous apparent modulus when tissue modulus is scaled from specimen CTattenuation. Journal of Biomechanics 37, 613-621.

Defresne, T. (1998). Segmentation techniques for analysis of bone by three-dimensional computed tomographic imaging. Technology and Health Care 6, 351-359.

Ding, M., Odgaard, A. \& Hvid, I. (1999) Accuracy of cancellous bone volume fraction measured by micro-CT scanning. Journal of Biomechanics 32, 323-326. 
Ding, M., Day, J.S., Burr, D.B., Mashiba, T., Hirano, T., Weinans, H., Sumner, D.R. \& Hvid, I. (2003) Canine Cancellous Bone Microarchitecture after one year of high-dose bisphosphonates. Calcified Tissue International 72, 737-744.

Ding, M. \& Hvid, I. (2000). Quantification of age-related changes in the structure model type and trabecular thickness of human tibial cancellous bone. Bone 26(3), 291-295.

Elmoutaouakkil, A., Peyrin, F., Elkafi, J. \& Laval-Jeantet, A.M. (2002). Segmentation of cancellous bone from high-resolution computed tomography images: influence on trabecular bone measurements. IEEE Transactions on Medical Imaging, 21(4),354-362.

Engelke, K., Graeff., W., Meiss, L. Hahn, M. \& Delling, G. (1993). High spatial resolution imaging of bone mineral using computed microtomography. Comparison with microradiography and undecalcified histologic sections. Investigative Radiology 28(4),341-349.

Feldkamp, L.A., Goldstein, S.A., Parfitt, A.M., Jesion, G. \& Kleerekoper, M. (1989). The direct examination of three-dimensional bone architecture in vitro by computed tomography. Journal of Bone and Mineral Research 4(1), 3-11.

Frey, P., Sarter, B. \& Gautherie, M. (1994). Fully automatic mesh generation for 3-D domains based upon voxel sets. International Journal of Methods in Engineering 37, 2735-2753.

Fyhrie, D.P. \& Hamid, M.S. (1993). The probability of distribution of trabecular level strains for vertebral cancellous bone. $39^{\text {th }}$ Annual Meeting of the Orthopaedic Research Society, 175.

Galante, J., Rostoker, W. \& Ray, R.D. (1970). Physical properties of trabecular bone. Calcified Tissue Research 5, 236-246.

Genant, H.K., Gordon, C., Jiang, Y., Lang, T.F., Link, T.M. \& Majumdar, S. (1999). Advanced imaging of bone macro and micro structure. Bone 25(1),149-152.

Gibson, L.J. (1985). The mechanical behavior of cancellous bone. Journal of Biomechanics 18, 317-328.

Goulet, R.W., Goldstein, S.A., Ciarelli, M.J., Kuhn, J.L., Brown, M.B. \& Feldkamp, L.A. (1994) The relationship between the structural and orthogonal compressive properties of trabecular bone. Journal of Biomechanics 27(4), 375-389.

Guo, X. E. (2001). Mechanical Properties of Cortical Bone and Cancellous Bone Tissue. Bone Mechanics Handbook, $2^{\text {nd }}$ Edition 10.9.

Harrigan, T.P., Jasty, M., Mann, R.W. \& Harris, W.H. (1988). Limitations of the continuum assumption in cancellous bone. Journal of Biomechanics 21, 269-275. 
Helterbrand, J.D., Higgs, R.E. Jr., Iversen, P.W., Tysarczyk-Niemeyer, G. \& Sato, M. (1997). Application of automatic image segmentation to tibiae and vertebrae from Ovariectomized rats. Bone 21(5), 401-409.

Hildebrand, T. \& Ruegsegger, P. (1997). Quantification of Bone Microarchitecture with the Structure Model Index. Computational Methods Biomechanics Biomedical Engineering 1(1), 15-23.

Hollister, S.J., Fyhrie, D.P., Jepsen, K.J. \& Goldstein, S.A. (1991). Application of homogenization theory to the study of trabecular bone mechanics. Journal of Biomechanics 24, 825-839.

Hollister, S.J., Brennan, J.M. \& Kikuchi, N. (1994) A homogenization sampling procedure for calculating trabecular bone effective stiffness and tissue level stress. Journal of Biomechanics 27(4), 433-444.

Jee, W.S.S. (2001). Integrated bone tissue physiology: anatomy and physiology. Bone Mechanics Handbook $2^{\text {nd }}$ Edition, CRC Press LLC, 1.1-1.34.

Keaveny, T.M., Borchers, R.E. Gibson, L.J. \& Hayes, W.C. (1993) Trabecular bone modulus and strength can depend on specimen geometry. Journal of Biomechanics 26 (8), 991-1000.

Keaveny, T.M., Pinilla, T.P., Crawford, R.P., Kopperdahl, D.L. \& Lou, A. (1997). Systematic and Random Errors in Compression Testing of Trabecular Bone. Journal of Orthopaedic Research 15, 101-110.

Kinney, J.H., Ryaby, J.T., Haupt, D.L. \& Lane, N.E. (1998). Three-dimensional in vivo morphometry of trabecular bone in the OVX rat model of osteoporosis. Technology and Health Care 6(5-6), 339-350.

Kinney, J.H., Lane, N.E. \& Haupt, D.L. (1995). In vivo, 3D-microscopy of trabecular bone. Journal of Bone Mineral Research 10, 264-269.

Kothari, M., Keaveny, T.M., Lin, J.C., Newitt, D.C., Genant, H. K. \& Majumdar, S. (1998). Impact of spatial resolution on the prediction of trabecular architecture parameters. Bone 22 (5), 437-4.43.

Kuhn, J.L. et al. (1990). Evaluation of a microcomputed tomography system to study trabecular bone structure. Journal of Orthopedic Research 8, 833-842.

Ladd, A.J.C. \& Kinney, J.H. (1998). Numerical errors and uncertainties in finite-element modeling of trabecular bone. Journal of Biomechanics 31, 941-945. 
Lane, N.E., Thompson, J.M., Haupt, D., Kimmel, D.B., Modin, G. \& Kinney, J.H. (1998). Acute changes in trabecular bone connectivity and osteoclast activity in the ovariectomized rat in vivo. Journal of Bone Mineral Research 13(2), 229-236.

Linde, F. \& Hvid, I. (1989). The effect of constraint on the mechanical behaviour of trabecular bone specimens. Journal of Biomechanics 22, 485-490.

Linde, F. \& Sorensen, H.C.F. (1993). The effect of different storage methods on the mechanical properties of trabecular bone, Journal of Biomechanics, 26, 1249.

Lorensen, W.E. \& Clne, H.E. (1987). Marching cubes: a high resolution 3D surface construction algorithm. Computer Graphics, 21(4), 163-169.

Maes, F., Vandermeulen, D. \& Suetens, P. (1999). Comparative evaluation of multiresolution optimization strategies for multimodality image registration by maximization of mutual information. Oxford University Press, Medical Image Analysis 3(4), 373-386.

Morita, M., Ebihara, A., Itoman, M. \& Sasada, T. (1994). Progression of osteoporosis in cancellous bone depending on trabecular structure. Ann Biomed Eng. 22(5), 532-9.

Müller, R., Van Campenhout, H., Van Damme, B., Van Der Perre, G., Dequeker, J., Hildebrand, T. \& Rüegsegger, P. (1998). Morphometric analysis of human bone biopsies: a quantitative structural comparison of histological sections and micro-computed tomography. Bone 23(1), 59-66.

Müller, R. \& Rüegsegger, P. (\|995). Three-dimensional finite element modeling of noninvasively assessed trabecular bone structures. Journal of Medical Engineering and Physics 17, 126-133.

Newman, E., Turner, A.S. \& Wark, J.D. (1995). The potential of sheep for the study of osteopenia: current status and comparison with other animal models. Bone 16(4 Suppl), 277S-284S. Review.

Niebur, G.L., Hsia, A.C., Chen, T.J. \& Keaveny, T.M. (1999). Simulation of trabecular bone yield using nonlinear finite element analysis. ASME IMECE, BED 43.

Odgaard, A. \& Linde, F. (1991). The underestimation of Young's modulus in compressive testing of cancellous bone specimens. Journal of Biomechanics 24, 691698.

Orr, T.E., Villars, P.A., Mitchell, S.L., Hsu, H.-P. \& Spector, M. (2001). Compressive properties of cancellous bone defects in a rabbit model treated with particles of natural bone mineral and synthetic hydroxyapatite. Biomaterials 22, 1953-1959. 
Otsu, N. (1979). A threshold selection method from gray-level histograms. IEEE Transactions on Systems, Man, and Cybernetics 9(1), 62-66.

Rho. J.Y., Ashman, R.B. \& Turner, C.H. (1993). Young's modulus of trabecular and cortical bone material: ultrasonic and microtensile measurements. Journal of Biomechanics 26, 111.

Rüegsegger, P., Koller, B. \& Müller, R. (1996). A microtomographic system for the nondestructive evaluation of bone architecture. Calcified Tissue International 58, 24-29.

Sharp, D.J., Tanner, K.E. \& Bonfield, W. (1990). Measurement of the density of trabecular bone. Journal of Biomechanics 23, 853-857.

Sivewright, G.J. \& Elliott, P.J. (1994). Interactive region and volume growing for segmenting volumes in MR and CT images. Medical Informatics 19(1), 71-80.

Stenström, M., Olander, B., Carlsson, C.A., Carlsson, G.A. \& Håkanson, R. (1995). Methodologic aspects of computed microtomography to monitor the development of osteoporosis in gastrectomized rats. Acad. Radiol. 9 (2),785-791.

Takahashi, M., Wehrli, F.W., Hilaire, L., Zemel, B.S. \& Hwang, S.N. (2002). In vivo NMR microscropy allows short-term serial assessment of multiple skeletal implications of corticosteroid exposure. PNAS 99 (7), 4574-4579.

Tanck, E., Homminga, J., Van Lenthe, G.H. \& Huiskes, R. (2001). Increase in bone volume fraction precedes architectural adaptation in growing bone. Bone 28(6), 650-654.

The American Heritage Dictionary $3^{\text {rd }}$ Edition. Copyright (C) (1994). Houghton Mifflin Company.

Turner, C.H. \& Burr, D.B. (2001) Experimental techniques for Bone Mechanics. Bone Mechanics handbook $2^{\text {nd }}$ Edition CRC Press LLC, 7.1-7.26.

Uchiyama, T., Tanizawa, T., Muramatsu, H. Endo, N., Takahashi, H.E. \& Hara, T. (1997). A morphometric comparison of trabecular structure of human illium between microcomputed tomography and conventional histomorphometry. Calcified Tissue International 61, 493-498.

Van Rietbergen, B., Weinans, H., Huiskes, R. \& Odgaard, A. (1995). A new method to determine trabecular bone elastic properties and loading using micromechanical finiteelement models. Journal of Biomechanics 28, 69-81.

Van Rietbergen, B., Majumdar, S., Pistoia, W., Newitt, D.C., Kothari, M., Laib, A. \& Rüegsegger, P. (1998). Assessment of cancellous bone mechanical properties from micro_FE models based on micro-CT, pQCT and MR images. Technology and Health Care IOS Press 6, 413-420. 
Van Rietbergen, B. Majumdar, S., Newitt, D. \& MacDonald, B. (2002). High-resolution MRI and micro-FE for the evaluation of changes in bone mechanical properties during longitudinal clinical trials: application to calcaneal bone in postmenopausal women after one year of idoxifene treatment. Clinical Biomechanics 17, 81-88.

Waarsing, J.H., Day, J.S. \& Weinans, H. (2004). An improved segmentation method for in vivo $\mu$ CT Imaging. Journal of Bone and Mineral Research 19(10), 1640-1650.

Wachter, N.J., Augat, P., Hoellen, I.P., Krischak, G.D., Sarkar, M.R., Mentzel, M., Kinzl, L. \& Claes, L. (2001). Predictive value of Singh index and bone mineral density measured by quantitative computed tomography in determining the local cancellous bone quality of the proximal femur. Clinical Biomechanics 16: 257-262.

Wolff, J. (1892). Das gestz der transformation der knochen, Berlin: A. Hirschwald (Springer-Verlag published an excellent English translation of this monography in 1986).

Yang, S., Hnat, W.P. \& Voor, M.J. (2005). A new voxel grayscale based 3D Image Registration Validation Method. XXth Congress of the International Society of Biomechanics and 29th Annual Meeting of the American Society of Biomechanics. Cleveland, Ohio.

Zhu, M., Keller, T.S. \& Spengler, D.M. (1994). Effects of specimen load-bearing and free surface layers on the compressive mechanical properties of cellular materials. Journal of Biomechanics 27, 57-66. 


\section{APPENDIX I}

\section{Program A}

clear

close all

"f program begins here"

for imnumber $=\#: \#$

s the file start from \# 10 \# close all

fpart='C: Iworklfolderlfile name';

" get files from specilic lacation

fpartout='C: \worklfolder fille name';

$r /$ sort new files to specific location

fname $\mathrm{l}=$ sprintf( $\% \mathrm{~s} \% \mathrm{~d} \% \mathrm{~s}^{\prime}$, fpart,imnumber)

fname2=sprintf( $\%$ s\%d\%s',fpartout,imnumber,'.tif')

$x x=$ imread(fname 1, 'tif'$\left.^{\prime}\right)$;

$\%$ read the image file

$\mathrm{z}(:,:)=\operatorname{xx}(:,:, 1)$;

B = imcrop(z,,[292 2201500 1300]);

\% choose the following parameters $\mid x$ min ymin width height in order to crop the images

imwrite(B,fname2,'tif','Compression','none');

1/, write the image file in tif format using the name as printed in fpartout and

"/ save it in the folder as printed in fpartout. tif is binary but compressed, in order

"/ to have non-compressed files, you need to add 'compression', 'none")

end

\% refers to selferelanatory motes 


\section{Program B}

clear

close all

for imnumber $=\#: \#$

C the tile start from \# 10 \#

close all

fpart='C: lwork $\backslash$ folderlfile name';

" get files from specitic location

fpartout='C: wworklfolder $\backslash$ file name';

r, sort new liles to specific location

fname $1=\operatorname{sprintf}\left(\% \mathrm{~s} \% \mathrm{~d} \% \mathrm{~s}^{\prime}\right.$, fpart,imnumber $)$

fname2=sprintf('\%s\%d\%s',fpartout,imnumber,'.tif')

$\mathrm{xx}=$ imread(fname 1, 'tif'$\left.^{4}\right)$;

\% read the image lile

$\mathrm{z}(:,:)=\mathrm{xx}(:,:, 1)$;

$\mathrm{C}=$ imrotate(z,185,'nearest','crop')

\% choose the degree of rotation in order to rotate the images imwrite(C,fname2,'tif','Compression','none');

$\%$ write the image file in tif format using the name as printed in fpartout and

\% save it in the folder as printed in fpartout. tif is binary but compressed, in

'/c order to have non-compressed fïles, you need to add 'compression'.'none')

end

ir refers to self-explanatory notes 


\section{Program C}

clear

close all

for imnumber $=161: 190$

close all

fpart='C:Iworklfolderlfile name';

fpartout='C:Iworklfolder file name';

fname l=sprintf('\%s\%d\%s',fpart,imnumber,'.tif')

fname $2=\operatorname{sprintf}(' \% \mathrm{~s} \% \mathrm{~d} \% \mathrm{~s}$, fpartout,imnumber,'.tif')

$x x=$ imread(fname 1, 'tif' $^{\prime}$;

$\mathrm{z}(:,:)=\mathrm{xx}(:,:, 1)$;

$\%$ I=graythresh $1(\mathrm{z})$;

$\%$ I=graythresh2(z);

/ Graythresh is a built in function in matlab but it comverts

$\%$ images to 8 bit before threshold. Graythreshl is a modified

/ version where it uses 16 bil images for threshold. Gaythresh 2 include

$\%$ idx 1 which allows you to add /f correction factor. Omit the $\%$ when need to use

graythresh 1 or 2 and otherwise.

BW = im2bw $(z, I)$;

\% max (max(BW) is 1 and picture : Black as 255 and white as 0

BW 1 =double(BW);

\% maximaxiBW I) is 1 and picture: Black as $(1$ and white as 255

$\operatorname{imshow}(z,[])$

/ show figure before threshold

figure, imshow(BW 1,[])

$\%$ show figure after after threshold

imwrite(BW 1,fname2,'tif','compression','none');

end

\section{Graythresh 1}

function level $=$ graythresh $(\mathrm{I})$

num_bins = 65536;

\% use 16 bit images to threshold

counts = imhist(I,num_bins $)$;

$\mathrm{p}=$ counts $/ \operatorname{sum}($ counts $)$

omega $=\operatorname{cumsum}(\mathrm{p})$;

$\mathrm{mu}=\operatorname{cumsum}\left(\mathrm{p} .{ }^{*}(1: \text { num_bins })^{\prime}\right)$;

mu_t $=$ mu(end);

state $=$ warning;

warning off;

sigma_b_squared $=($ mu_t $*$ omega $-\mathrm{mu}) .^{\wedge} 2 . /\left(\right.$ omega.$^{*}(1$ - omega $\left.)\right)$; 
warning(state);

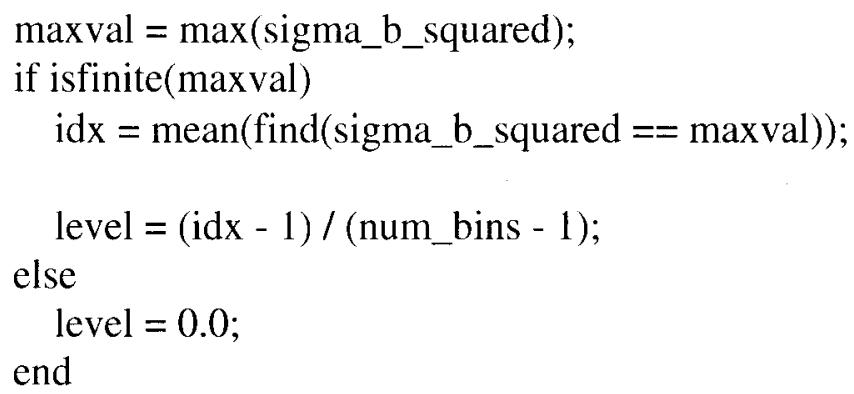

\section{Graythresh 2}

function level $=$ graythresh $(\mathrm{I})$

num_bins $=65536$;

counts = imhist(I,num_bins $)$;

$\mathrm{p}=$ counts $/$ sum(counts);

omega $=\operatorname{cumsum}(\mathrm{p})$;

$m u=\operatorname{cumsum}\left(\mathrm{p} . *(1: \text { num_bins })^{\prime}\right)$;

$\mathrm{mu} \mathrm{t}=\mathrm{mu}(\mathrm{end})$;

state $=$ warning;

warning off;

sigma_b_squared $=($ mu_t $*$ omega $-\mathrm{mu}) .^{\wedge} 2 . /\left(\right.$ omega.$^{*}(1-$ omega $\left.)\right) ;$

warning(state);

maxval $=\max ($ sigma_b_squared $)$;

if isfinite(maxval)

$\mathrm{idx}=$ mean(find(sigma_b_squared $==\operatorname{maxval})$ );

id $1=\mathrm{idx}+\#$;

$\%$ addition of"; correction factor

$$
\text { level }=(\text { idx } 1-1) /(\text { num_bins }-1) \text {; }
$$

else

level $=0.0$;

end

\% refers to self-explanalory notes 


\section{Program D}

clear

close all

fid = fopen('filename.txt','a+');

', open a file name to stere all the bone material from the thresholded inages for imnumber = \#:\#

ot the like start from in \#

close all

fpart='C: Idirectory\subdirectorylfilename';

fname $1=$ sprintf('\%s\%d\%s',fpart,imnumber,'.tif')

t, the lile start from \# io \# atulomaticalls

$\mathrm{xx}=\mathrm{imread}\left(\right.$ fname 1, 'tif' $^{\prime}$;

t/s read images

$\mathrm{z}(:,:)=\mathrm{xx}(:,:, 1)$;

threshold=215;

"t the number 255 can be any bing above $\theta$ since the images were previously

for $\mathrm{r}=2: 101$;

C/ lines of rows which refers toy

for $\mathrm{c}=2: 101$;

r lines of columms which refers $t 0 x$

if $\mathrm{z}(\mathrm{r}, \mathrm{c})>$ threshold

$\mathrm{x}=\mathrm{c}$;

$\mathrm{y}=\mathrm{r}$;

$\mathrm{k}=$ imnumber;

fprintf(fid,' \%g ',x);

fprintf(fid,' $\% \mathrm{~g}$ ',y);

fprintf(fid,'\%g', rın',k);

$\%$ print all the bone material information in the $t \times 1$ file.

end

end

end

end

fclose(fid);

fid = fopen('filename.txt','r');

fid 1 = fopen('count_filename.txt', 'a+');

', open another file to store the number of bone pixels from filename.ts

count $=0$;

while $\sim$ feof(fid)

line $=$ fgetl(fid);

if isempty(line) $\mid$ strncmp(line,' $\%$ ',1)

continue

end

count $=$ count +1

or count all the bone pivels 
end

fprintf(fid 1,'\%d lines',count);

', print and save number (bone volume) in the count hlename. $x$ t.

$\mathrm{TF}=$ bone volume / total volume can be easily calculated

fclose(fid1);

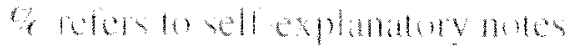

\section{Program E}

clear

close all

fid $=$ fopen $(", ' r+')$;

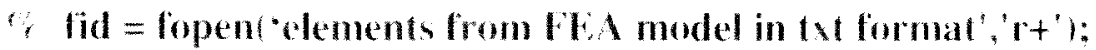

for imnumber $=1^{\text {st }}$ number:last number

\% lor imnumber $=1$ " number: ast number of the bone images

close all

fpart='c:|worklfolderlfile name';

fname $1=$ sprintf('\%s\%d\%s',fpart,imnumber,'.tif')

$\mathrm{xx}=$ imread(fname 1, 'tif' $^{\prime}$;

$\mathrm{z}(:,:)=\mathrm{xx}(:,:, 1)$;

/e 2 representing the matrix in row and colum

threshold=255;

/c the number 255 can be any thing above $\theta$ since the images were previously thresholded. It is just looking for all the white pixels representing bone.

for $\mathrm{r}=1^{\text {st }}$ row: last row;

for $\mathrm{c}=1^{\text {st }}$ column: last column;

$\% r=y, c=x$

tline $=$ fgets(fid);

\%o to the row that corresponds to the column

disp(tline)

co display the row and column

if $z(r, c)>$ threshold;

\% It is extracting all the elements representing bone tissue that match the

\% corresponding row and column of bone pixels from each image and eliminating

\% all the non-bone tissue elements

fid1 = fopen('give a file name with txt extension,'a+');

/c at means keep adding all the information

fprintf(fid1,tline);

Crave all the elements representing bone tissue

fclose(fid1);

end

end

end

end

\% refers to self-explanatory moles 


\section{DATA}

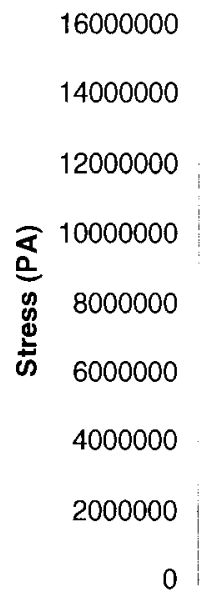

$0 \quad 0.002$

0.004

0.006

0.008

$y=1.228 E+09 x-2.041 E+06$

$R^{2}=9.993 E-01$

Strain

Rabbit Bone 721LL
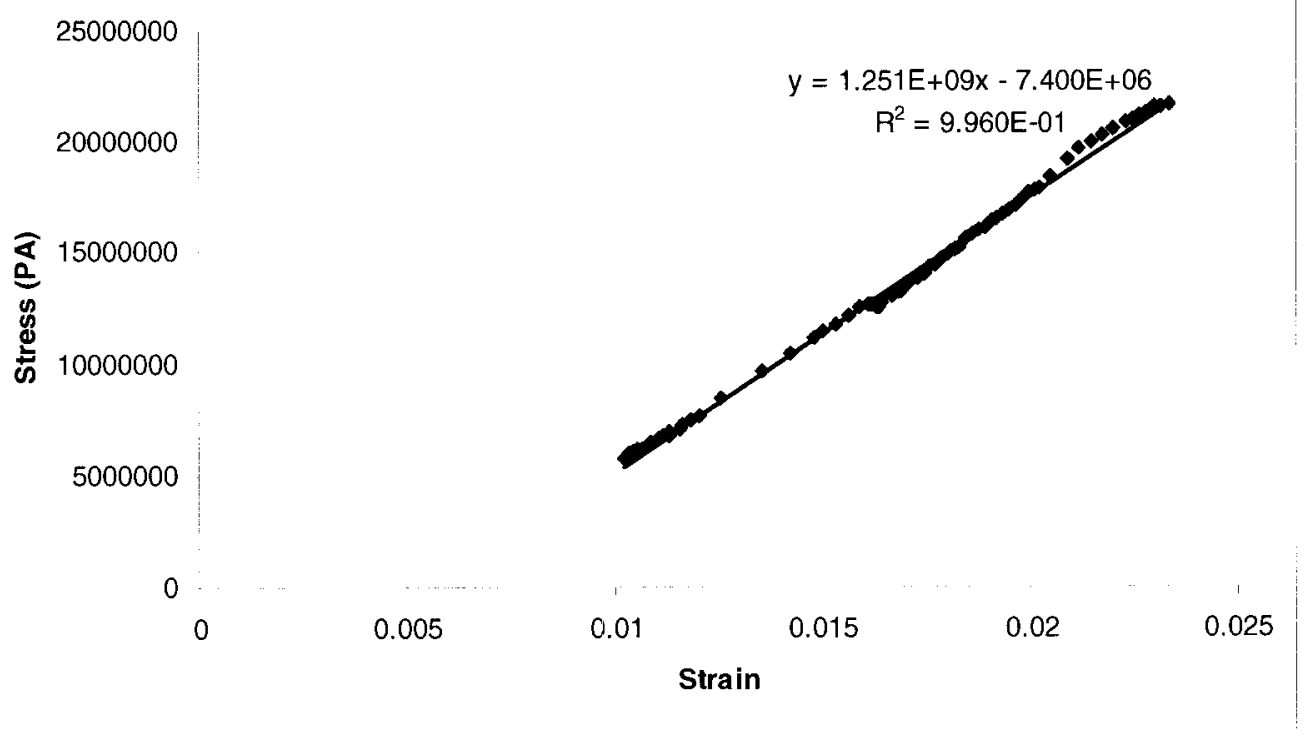

Rabbit Bone 721LM 


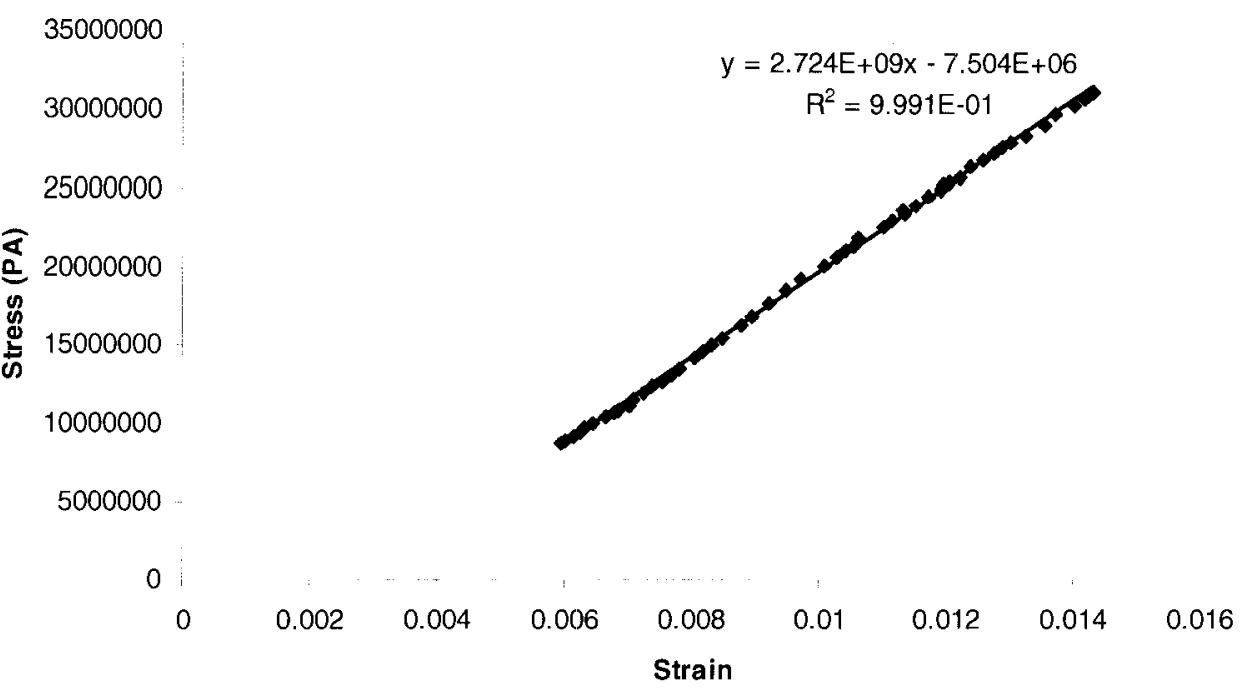

Rabbit Bone 720LL

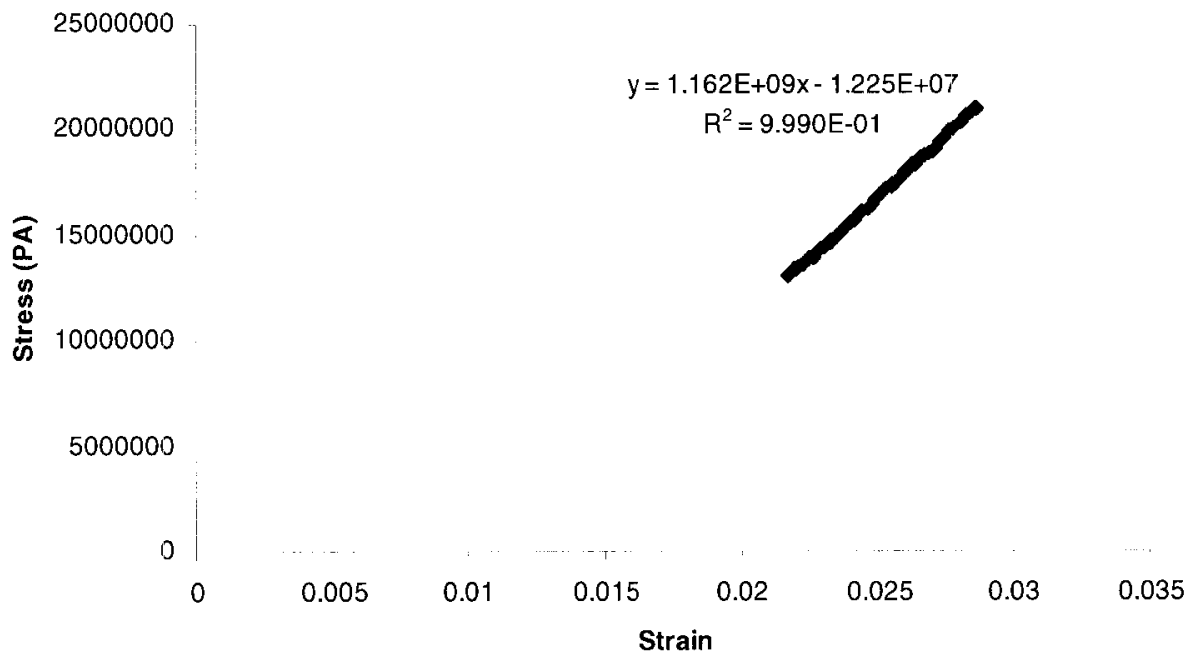

Rabbit Bone 720LM 

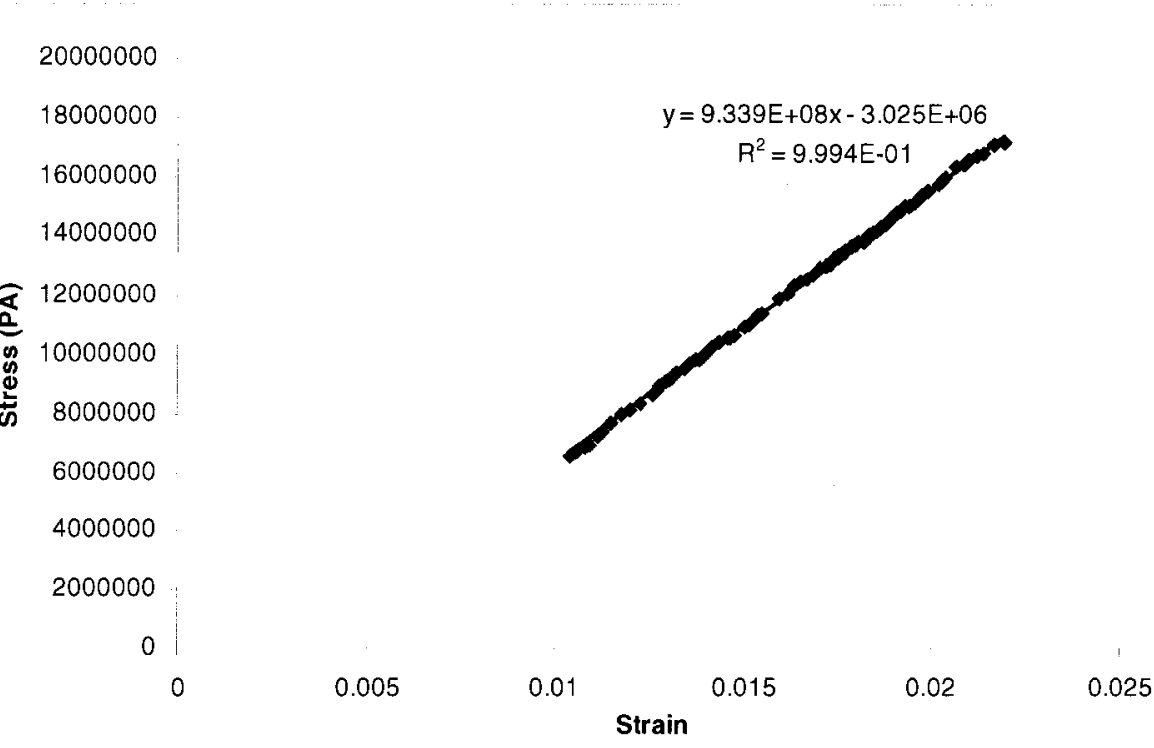

Rabbit Bone 718RL

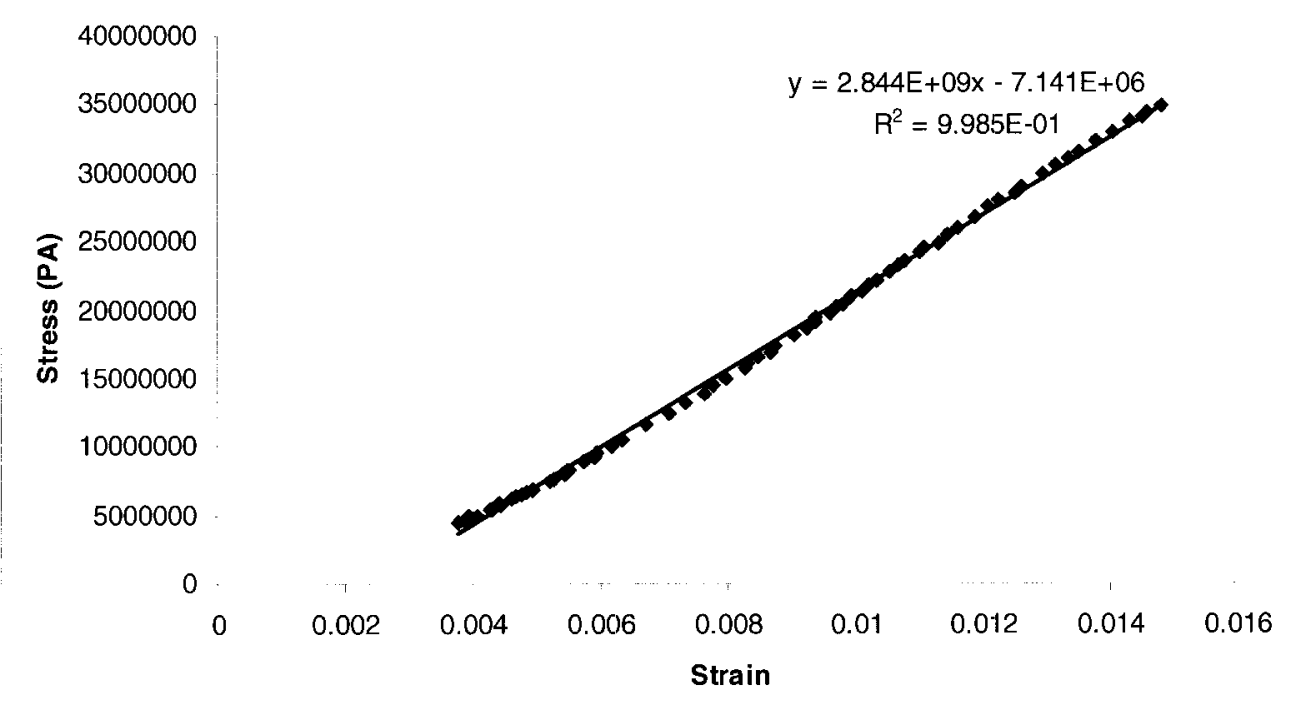

Rabbit Bone 718RM 


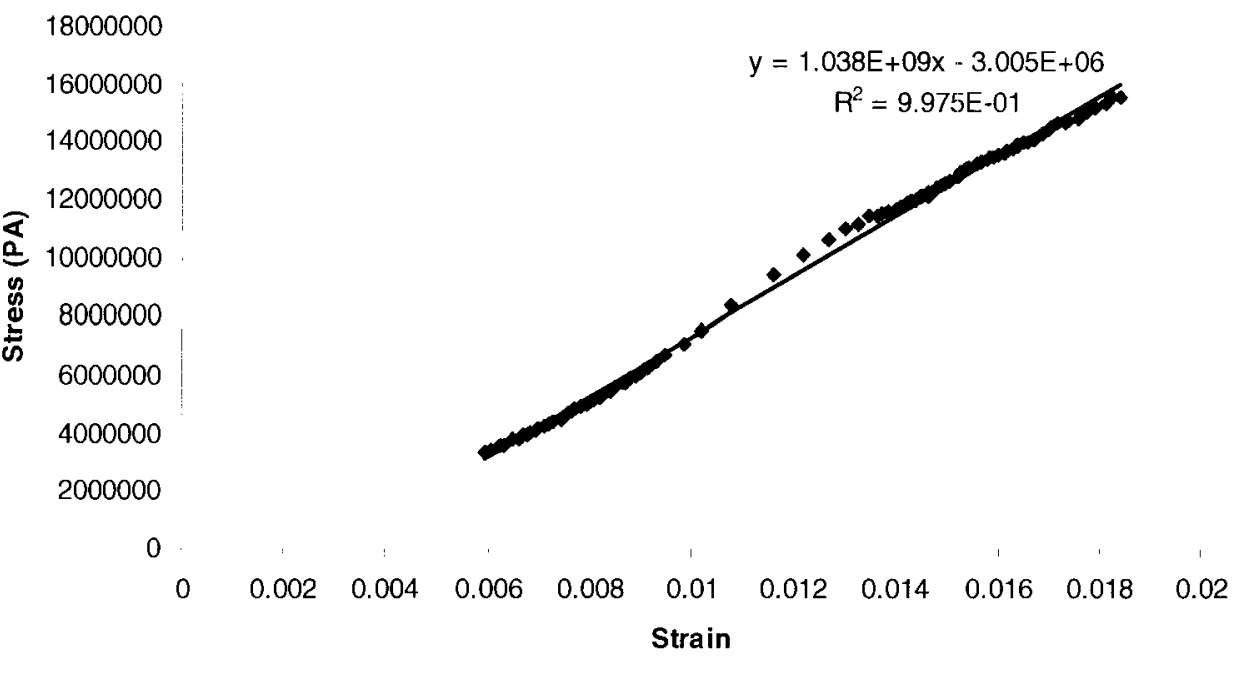

Rabbit Bone 762RL

30000000

25000000

$y=2.234 E+09 x-1.686 E+07$

$\mathrm{R}^{2}=9.993 \mathrm{E}-01$

$\tau^{20000000}$

के

of 15000000

紊

10000000

5000000

0

0

0.005

$\begin{array}{lll}0.01 & 0.015 & 0.02 \\ & \text { Strain }\end{array}$

0.025

Rabbit Bone 762RM 


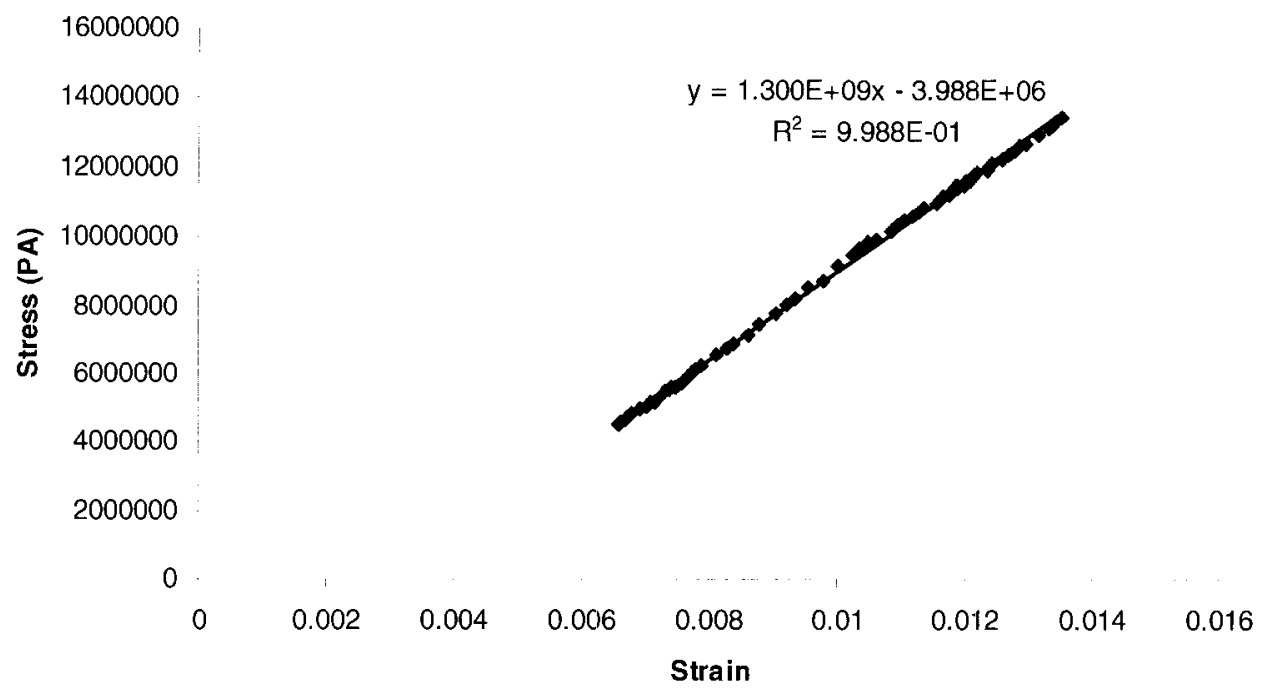

Rabbit Bone 751LL

25000000

20000000

$$
\begin{gathered}
y=2.824 E+09 x-6.753 E+06 \\
R^{2}=9.992 E-01
\end{gathered}
$$

दิ 15000000

के 10000000

5000000

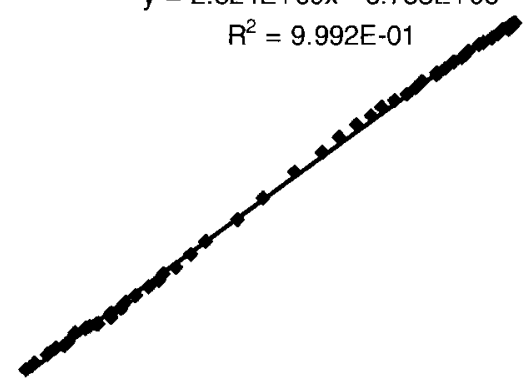

0

0

0.002

0.004

0.006
Strain

0.008

0.01

0.012

\section{Rabbit Bone 751LM}




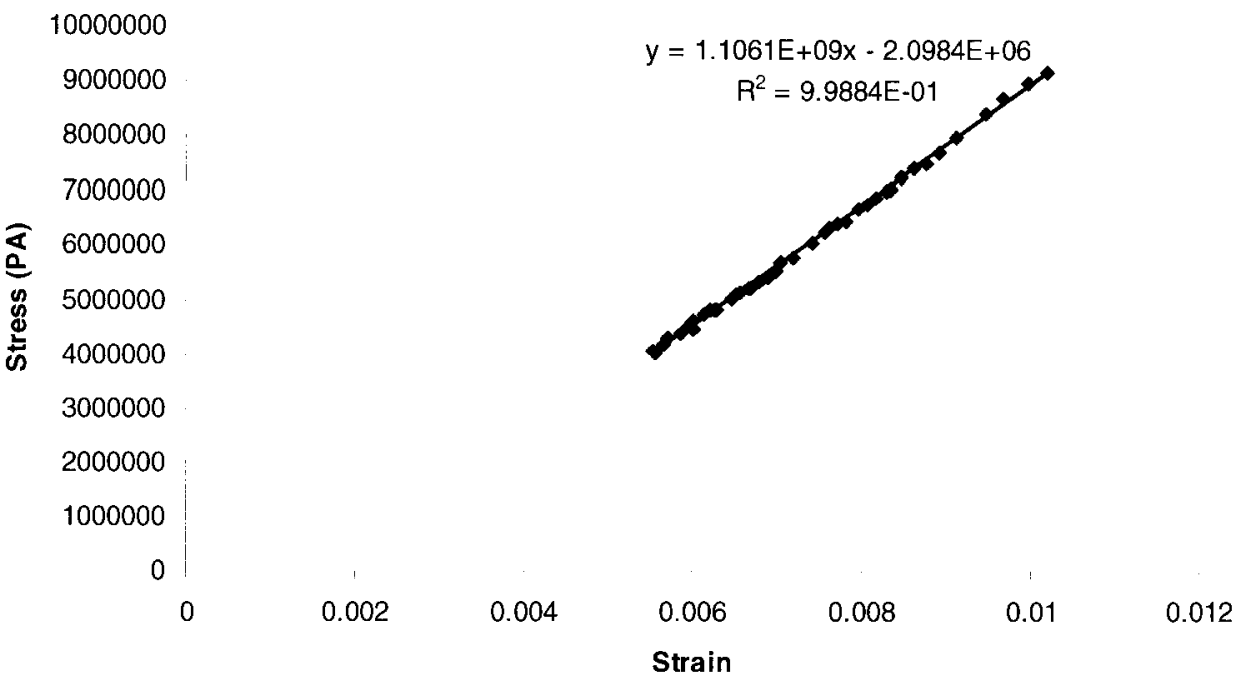

Rabbit Bone 750RL

30000000

25000000

$y=2.146 E+09 x-9.772 E+06$

$R^{2}=9.995 E-01$

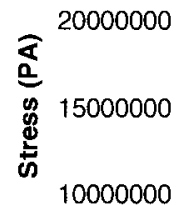

5000000

0

0

$0.002 \quad 0.004$

0.006

0.008

$0.01 \quad 0.012$

$0.014 \quad 0.016$

0.018

Strain

Rabbit Bone 750RM 


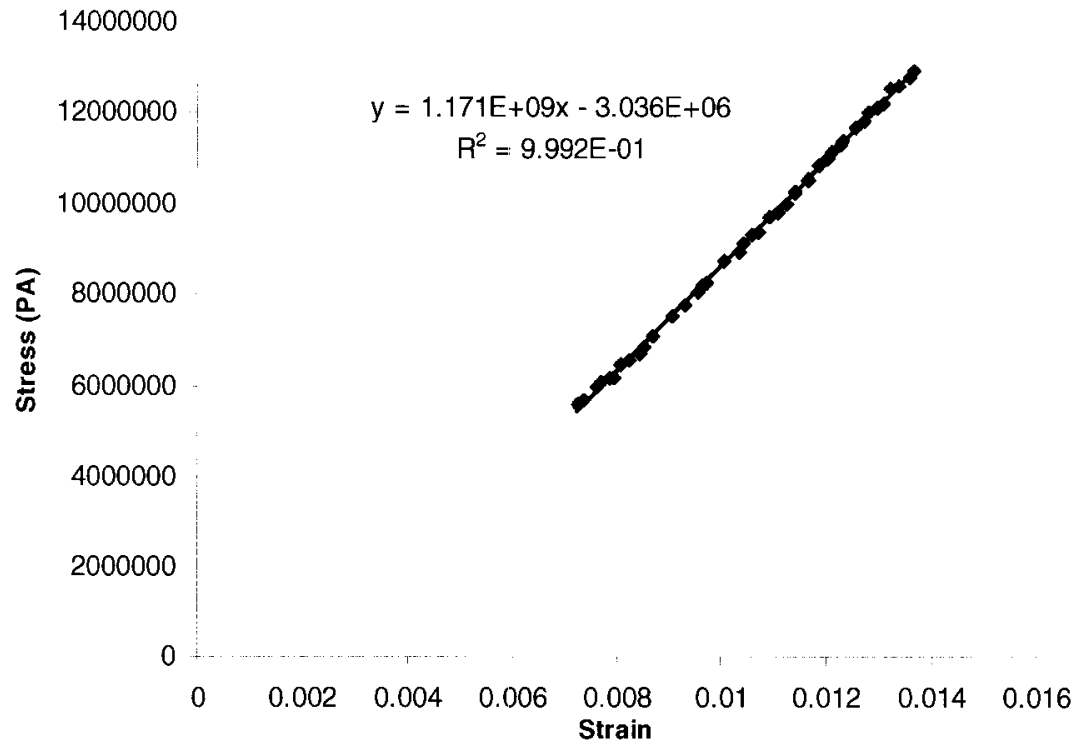

Rabbit Bone 719LL 


\section{CURRICULUM VITAE}

NAME:

ADDRESS:

EDUCATION:
Boon Horng Kam

Department of Mechanical Engineering

Orthopedic Bioengineering Laboratory

University of Louisville

500, South Preston St.

Instructional Building B - R 128

Louisville, KY 40202, U.S.A.

Associate Degree, Engineering, Mertajam Community College, Penang, Malaysia

1992-1994

B.Sc. (Hons), Bioengineering, School of Agricultural and Bioengineering Mississippi State University, MsState, U.S.A.

$1995-1996$

MEng, Mechanical Engineering

with emphasis on biomechanical

School of Mechanical and Aerospace Engineering

Nanyang Technological University, Singapore.

$1999-2000$

Ph.D., Mechanical Engineering

with emphasis on biomechanics,

University of Louisville

Kentucky, U.S.A.

$2000-2005$

LEADERSHIP TRAINING: Future Professors Program

Department of Education,

University of Louisville, Louisville

Kentucky, U.S.A

2003-2004 
Graduate Research Assistant

Orthopedic Bioengineering Laboratory,

University Hospital, University of Louisville,

Kentucky, USA

$2003-2005$

- Plan and conduct research projects and maintain database and statistical support.

- Utilized, learned, designed, modified, developed and conducted bone preparation, mechanical testing and other experimental methods i.e. Archimedes and serial milling of cancellous bone.

- Prepared chemical solutions and conducted experiments i.e. cleaning, staining and embedding of trabecular bone

- Involved and conducted scanning activities of in vivo and in vitro distal femur of rabbits.

- Used FEA (Abaqus) program in design and develop virtual mechanical testing which to be compared with traditional mechanical testing.

- Developed programs with Matlab software for measuring bone volume fraction and thresholding, and for abstracting bone information from micro CT files to FEA (Abaqus) and vice versa.

- Collect and analyze detailed architecture of trabecular bone using high-resolution micro-CT scans using custom written Matlab program and FEA.

- Prepared manuscripts for submission to journals and conferences.

- Conducted animal surgery: ovariectomy(OVX) and sham OVX on 18 white New Zealand rabbits.

- Also participated in project \#3 - create osteoporosis in the rabbit femur with steroid (scenario 1) and low calcium diet (scenario 2), Analyse accordingly.

Research Scientist

Department of Experimental Surgery

Singapore General Hospital

Singapore

2002 (sabbatical)

Orthopaedic Research:

- Plan and conduct research projects and maintain database and statistical support.

- Collect and analyze data from completed experiments.

- Worked on cadaveric specimens

- Prepare manuscripts for submission to journals and conferences.

Orthopaedic research projects include the following:

1. Kinematics of Knee Joint : An experimental Study on 16 human cadavers. This was a collaboration project with Dr Kim Wang Do, Assoc. Professor from Singapore Nanyang Technological University (NTU), Dr Wong YueShuen, Orthopaedic Surgeon from Singapore Alexandra Hospital and Madam Ying Ning, a PhD candidate from NTU. Please refer to publication [Project completed within my service] 
2. Kinematics of Lumbar Spine: An experimental Study on identifying the local motion of lumbar spine segments. Only 5 human cadaver spines were allocated for this study. This was a collaboration project with Dr Tan Soon Huat. [Project completed within my service]

3. Compression tests of cancellous lumbar spine vertebrae: An experimental Study on 6 human cadaver lumbar spines vertebrae. This was a project conducted with a group of 3 undergraduate students in their final year project Collaborated with Dr Tan Soon Huat, Assoc. Professor from Singapore Nanyang Technological University (NTU).

[First part of the project completed within my service] All data is securely detained with Dr Tan Soon Huat for further research in NTU.

4. Mechanical Testing on Spine wires and cables: An initial tests of engineering wires were mechanically tested in order to obtain the best method before testing the spine wires and cables. A collaboration project with Dr Tan Seang Beng, Head of Orthopaedic Surgery Department, Singapore General Hospital and Dr Chou Siaw Meng, Assoc. Professor from Singapore Nanyang Technological University (NTU).

[First part of the project completed within my service] All data is securely detained with Dr Chou Siaw Meng for further research in NTU]

\title{
Experimental surgery work:
}

1. white mouse surgery with Prof. Li, General Surgeon.

2. assisted in skull workshop setup with Mr Robert Ng, Lab Manager.

3. porcine surgery (parachute anastomosis between the bile duct and deodonum) with Dr Johanna Laukkarinen, Surgeon from Finland.

4. histology work: samples prepared using microtome and warm water bath with Irene Kee, Senior Technologist.

5. animal(porcine) wound management with Irene Kee.

6. Biochemistry work: performed bile acids test, phospholipids measurements and $\beta$ glucuronidase tests with Irene Kee.

\author{
Graduate Research Assistant \\ Orthopaedic Research Laboratory, \\ University Hospital, University of Louisville, \\ Kentucky, USA \\ $2000-2001$
}

- Planned and conducted research projects and maintained database and statistical support. Collect and analyze data from completed experiments. Prepare only the materials and methods section of manuscripts for submission to journals.

- The summary of research projects in biomechanical study is as follows: 
- Involved in fracture fixation study - Compared 4 types of external fixation frames on fiberglass bone, tibia in particular. Location of half pins and wires remained as reference for all frames. Frames include DePuy Ace, EBI, Synthes and Hoffman. Frames were configured as hybrid fixation with half pins on the distal and wires connected to rings on the proximal of the tibia. Testing done on MTS machine. Axial and Torque testing method were applied. All testing jigs were custom designed and manufactured.

- Participated in animal (goat) surgery in the study of impaction bone grafting of hip replacement using defatted and non-defatted morselized bone.

- Involved in the study of methods of application using hydroxyapetite bone cement particularly in hip replacement.

Graduate and Undergraduate research work

Mississippi State University, USA

$1995-1996$

\section{Artificial Encapsulation of A Total Hip Prostheses}

In an attempt to improve long term performance of a total hip prosthesis and eliminate the adverse effects of wear debris, silicone elastomer was proposed as the artificial encapsulation of a total hip prosthesis. The research involved soaking the material in a pseudo physiological solution for a period of $0,3,6$, and 9 weeks. Thus, the material was tested using an instron machine. The standard testing methods: tensile properties testing and tear strength testing in accordance to the American Standard Testing Society (ASTM). Based on these experimental results, silicone elastomer is a viable capsule material for the encapsulation of a total hip prosthesis.

\section{Biosystem Simulation Modeling}

Vissim 2.0 is a modeling package that was used in biosystem simulation modeling. Created a model, which filled a container with water, heated it to $100 \mathrm{C}$, emptied the container, and automatically started the process over. Introduced to stochastic modeling. Take for example, Walmart Supermarket. This model was an attempt to simulate the manner in which people enter and exit Walmart. $2^{\text {nd }}$ model: a predator-prey model. The predator should search methodically for the prey. The process of moving toward the prey was complicated because the prey would not necessarily stand still. Therefore, the final step was developed which is the kill. Other than the above models, modeling exercises were also carried out on a model which to maintain the level of sugar content in diabetic patients, space world model, and finally, missile and warhead model. 
3. Purification of Amylase and Amylopectinase Enzymes from Ruminobacter Amylophilus

Ruminobacter Amylophilus can only utilize starch and maltodextrins as an energy substrate. It makes an excellent model for physiological studies of starch degradation. This project focused on purification and characterization of the amylase and amylopectinase enzymes from R. Amylophilus, both membrane and soluble. I was involved in all aspects of this study, including the use of protein chromatography, isoelectric focusing, buffer preparation, anaerobic media preparation, and cultivation of anaerobic bacteria. I spent an average of 4-6 hours per week working in the microbiology science laboratory, Harned Biology Building, Miss State University, U.S.A.

WORK EXPERIENCE: Product Executive

Orthopaedic And Trauma surgery,

DePuy c/o Boehringer Mannheim (M) Sdn Bhd.

Malaysia

$1996-1998$

- Worked closely with orthopaedic surgeons and nurses in the operating theatre

- Attended product training - Hip, Knee, Spine \& Trauma (Basic, Intermediate \& Advance) provided by DePuy Singapore, DePuy Australia \& DePuy International.

- Planning for hip, knees, trauma and spine surgeries

- Performed bioskills workshops (implants and instrumentations) on hip replacement, knee replacement, trauma and spine surgery

- Attended hips ( 150 cases), knees (300 cases), trauma (50 cases), and spine (75 cases) surgeries in Malaysia.

- Interacted closely with product managers internationally for new products, existing products and technical product support.

- Responsible for logistics and management in products and instrumentation inventory.

TEACHING: $\quad$ English Tutor

Dunville Language Training Center,

Penang, Malaysia

1994

Worked 45 hours per week after completing Associate Arts Degree in engineering in Mertajam Community College, Penang, Malaysia. Taught (conversational English, Business Communication English, Public Speaking, and Presentation) a group of 20-30 students varies from secondary level, colleges, factories (e.g. Hitachi, Otis, Motorola, Trane, etc) 
Science Laboratory Assistant

Mertajam Community College

Penang, Malaysia

1992-1993

Worked 20 hours per week. Assisted Professor Seng from University Science Malaysia (USM) in chemical solution preparation, physics, and biology experiments for grades 11 , 12, and freshmen year in the American Degree Program. Carried out a class of 40 students for the above mentioned science experiments in the science laboratory sessions. Proctored a class of 10 during final exam for higher certificate government examination in the experimental lab (Biology, Physics, and Chemistry).

SERVICE: $\quad$ Tutoring and Homework (Math and Reading)
2 Hours Weekly $3^{\text {rd }}-7^{\text {th }}$ Grades
Corinthian Christian Life Center
Louisville, Kentucky, USA
2004

Volunteer for special activities

The Kids of Kosair Charities

Louisville, Kentucky, USA

Tutoring and Homework (Math and Reading)

2 Hours Weekly $\quad 7^{\text {th }}-10^{\text {th }}$ Grades

Malaysian Buddhist Community

Georgetown, Penang, Malaysia

1993-1994

\section{PUBLICATIONS:}

Kam BH, Chou SM and Tan SB. Comparative Biomechanics: Cervical Spine Facet Joint versus Lateral Mass Screw Insertion Technique. JOURNAL OF MUSCULOSKELETAL Accepted in October 2005 (In Press)

Voor MJ, Kam BH, Antoci V and Roberts C. Hybrid External Fixation of Proximal Tibia Fractures: Biomechanical Analysis of Four Commercially Available Systems. ORTHOPEDICS. Accepted in September 2005 (In Press)

Ying Ning, Kim Wangdo, Wong Yueshuen and Kam BH. Analysis of Passive Motion Characteristics of the Ankle Joint Complex Using Dual Euler Angle Parameters. JOURNAL OF CLINICAL BIOMECHANICS. Accepted in August 2003 and Published (can be viewed online). 


\section{INTERNATIONAL AND NATIONAL CONFERENCES:}

Kam BH, Voor MJ, Antekeier SB, Yang S, Waddell SW and Burden RL Jr. In Vivo Micro CT Scanning of a Rabbit Distal Femur. XXth ISB and $29^{\text {th }}$ ASB July 2005. Accepted and presented.

Kam BH, Yang S, Voor MJ, Antekeier SB, Waddell SW and Burden RL Jr. In Vivo Computational and Virtual Mechanical Techniques in Quantifying Trabecular Structure using Micro CT. Pre-ORS March 2004. Presented.

Kam BH, Tan SB and Chou SM. Morphological Study of the Cervical Spine on 88 Lateral X-rays. $12^{\text {th }}$ International Congress on Biological and Medical Engineering 2003. Presented.

Kam BH, Tan SB and Chou SM. Strength Studies of Posterior Cervical Spinal Systems. $10^{\text {th }}$ International Congress on Biological and Medical Engineering 2000, 359-eoa. Accepted.

\section{SCIENTIFIC PRESENTATIONS:}

Kam BH, Voor MJ, Antekeier SB, Waddell SW and Burden RL Jr. In Vivo Micro CT to quantify osteoporosis in the rabbit distal femur. Research Louisville Symposium, Nov 2003. Presented

Proficient in the following: Matlab 6.5 and Abaqus 5.0 Ansys 4.5 and Pro Engineering 2.0

Microsoft Office i.e. Word, Excel, Powerpoint 